\section{Electronics and Electrical Engineering Laboratory}

\section{Technical Progiress Bulletin}

Covering Laboratory Programs, October to December 1991, with 1992/1993 EEEL Events Calendar

\section{J. A. Gonzalez Compiler}

\author{
U.S. DEPARTMENT OF COMMERCE \\ Technology Administration \\ National Institute of Standards \\ and Technology \\ Electronics and Electrical \\ Engineering Laboratory \\ Semiconductor Electronics Division \\ Gaithersburg, MD 20899
}

July 1992 



\section{Electronics and Electrical \\ Engineering Laboratory}

\section{Technical \\ Progiress \\ Bulletin}

\section{J. A. Gonzalez Compiler}

Covering Laboratory Programs, October to December 1991, with 1992/1993 EEEL Events Calendar

\section{1-4}

U.S. DEPARTMENT OF COMMERCE Technology Administration National Institute of Standards and Technology Electronics and Electrical Engineering Laboratory Semiconductor Electronics Division Gaithersburg, MD 20899

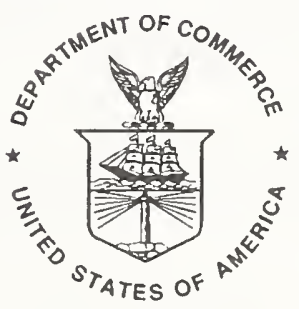

U.S. DEPARTMENT OF COMMERCE Barbara Hackman Franklin, Secretary 


\section{INTRODUCTION TO JULY 1992 ISSUE OF THE EEEL TECHNICAL PROGRESS BULLETIN}

This is the thirty-seventh issue of a quarterly publication providing information on the technical work of the National Institute of Standards and Technology Electronics and Electrical Engineering Laboratory (EEEL) (until February 1991, the Center for Electronics and Electrical Engineering). This issue of the EEEL Technical Progress Bulletin covers the fourth quarter of calendar year 1991.

Organization of Bulletin: This issue contains abstracts for all relevant papers released for publication by NIST in the quarter and citations and abstracts for such papers published in the quarter. Entries are arranged by technical topic as identified in the Table of Contents and alphabetically by first author under each subheading within each topic. Unpublished papers appear under the subheading "Released for Publication." Papers published in the quarter appear under the subheading "Recently Published." Following each abstract is the name and telephone number of the individual to contact for more information on the topic (usually the first author). This issue also includes a calendar of Laboratory conferences and workshops planned for calendar year 1992/1993 and a list of sponsors of the work.

Electronics and Electrical Engineering Laboratory: EEEL programs provide national reference standards, measurement methods, supporting theory and data, and traceability to national standards. The metrological products of these programs aid economic growth by promoting equity and efficiency in the marketplace, by removing metrological barriers to improved productivity and innovation, by increasing U.S. competitiveness in international markets through facilitation of compliance with international agreements, and by providing technical bases for the development of voluntary standards for domestic and international trade. These metrological products also aid in the development of rational regulatory policy and promote efficient functioning of technical programs of the Government.

The work of the Laboratory is conducted by four technical research Divisions: the Semiconductor Electronics and the Electricity Divisions in Gaithersburg, Md., and the Electromagnetic Fields and Electromagnetic Technology Divisions in Boulder, Colo. In 1991, the Office of Law Enforcement Standards, formerly the Law Enforcement Standards Laboratory, was transferred to EEEL. This Office conducts research and provides technical services to the U.S. Department of Justice, State and local governments, and other agencies in support of law enforcement activities. In addition, the Office of Microelectronics Programs (OMP) was established in EEEL to coordinate the growing number of semiconductor-related research activities at NIST. Reports of work funded through the OMP are included under the heading "Semiconductor Microelectronics."

Key contacts in the Laboratory are given on the back cover; readers are encouraged to contact any of these individuals for further information. To request a subscription or for more information on the Bulletin, write to EEEL Technical Progress Bulletin, National Institute of Standards and Technology, Metrology Building, Room B-358, Gaithersburg, MD 20899 or call (301) 975-2220.

Laboratory Sponsors: The Laboratory Programs are sponsored by the National Institute of Standards and Technology and a number of other organizations, in both the Federal and private sectors; these are identified on page 46.

Note on Publication Lists: Publication lists covering the work of each division are guides to earlier as well as recent work. These lists are revised and reissued on an approximately annual basis and are available from the originating division. The current set is identified in the Additional Information section, page 44 . 
Silicon Materials .......................................... 2

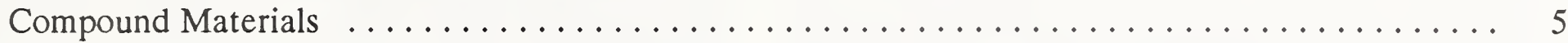

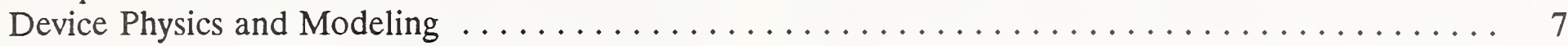

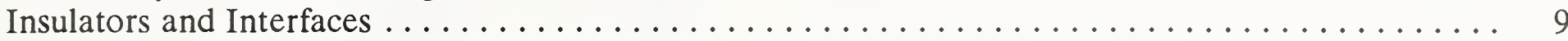

[For Separation by Implanted Oxygen (SIMOX) and for

Silicon-on-Insulator (SOI) see Silicon Materials]

Dimensional Metrology ..................................... 11

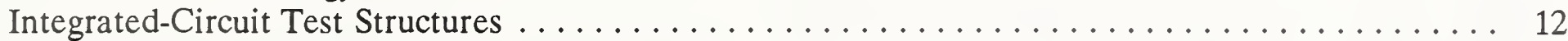

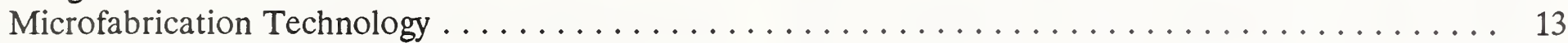

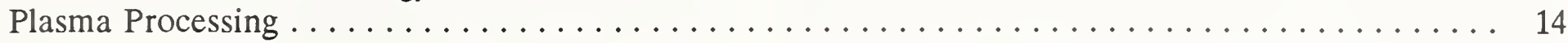

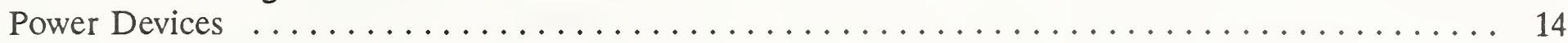

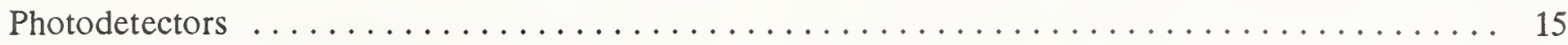

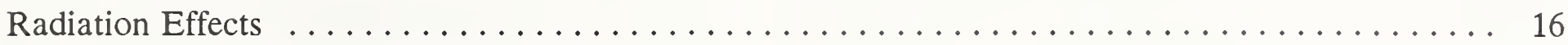

Other Semiconductor Metrology Topics $\ldots \ldots \ldots \ldots \ldots \ldots \ldots \ldots \ldots \ldots \ldots \ldots \ldots \ldots \ldots$

SIGNAL ACQUISITION, PROCESSING, AND TRANSMISSION $\ldots \ldots \ldots \ldots \ldots \ldots \ldots \ldots \ldots$

DC and Low-Frequency Metrology . . . . . . . . . . . . . . . . . . . . . . . . . 17

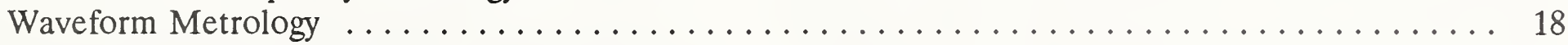

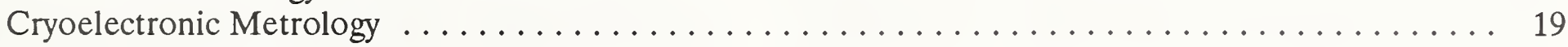

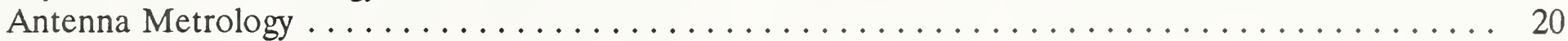

[Also see Electromagnetic Interference - Radiated]

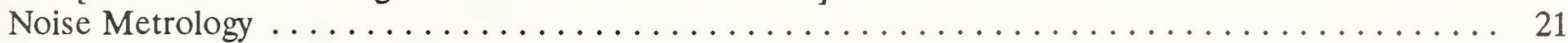

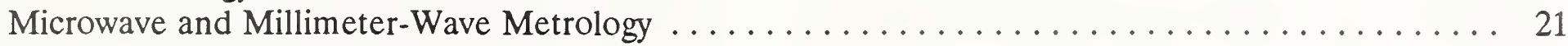

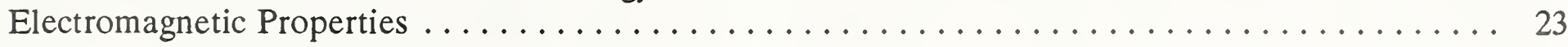

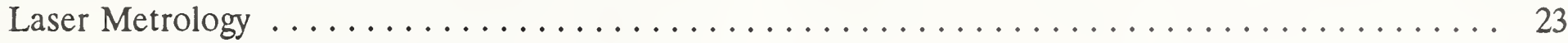

Optical Fiber Metrology ..................................... 23

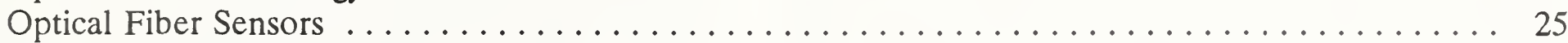

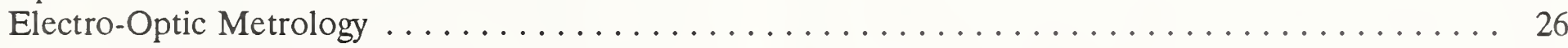

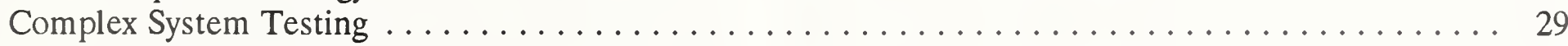

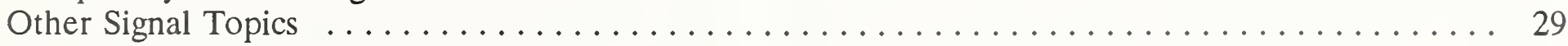

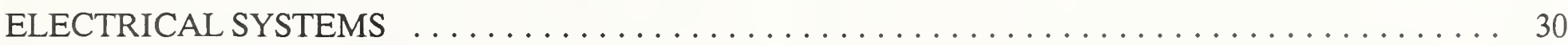

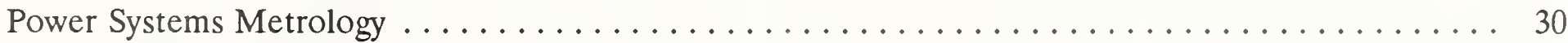

Magnetic Materials and Measurements . . . . . . . . . . . . . . . . . 35

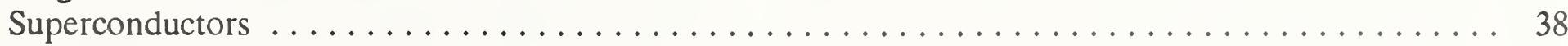

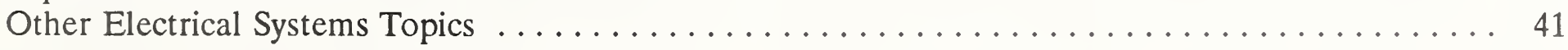

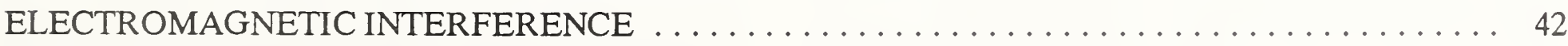

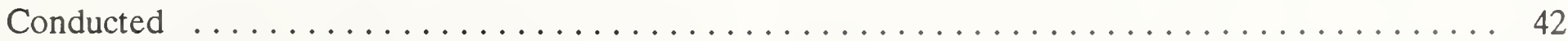

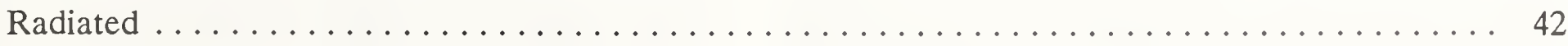

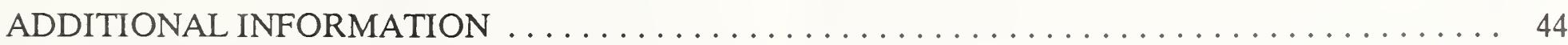

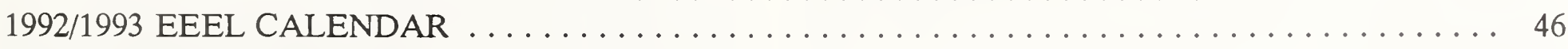

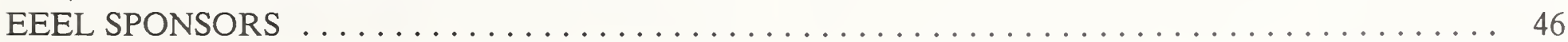

KEY CONTACTS IN LABORATORY, LABORATORY ORGANIZATION . . . . . . . inside back cover 


\section{FUNDAMENTAL ELECTRICAL MEASUREMENTS}

\section{Released for Publication}

Martinis, J.M., Zimmerli, G., Eiles, T.M., Jensen, H.D., and William, E., A New Measurement of the Fine Structure Constant Utilizing a Coulomb Blockage Charge Pump, to be published in the CPEM Digest, Conference on Precision Electromagnetic Measurements, Paris, France, June 9-12, 1992.

We propose an experiment from which a new measurement of the fine structure constant can be obtained. This measurement utilizes an electron pump device that can transfer a countable number of electrons into a capacitor which then can be compared with the calculable capacitor. We discuss key requirements of the experiment.

[Contact: John M. Martinis, (303) 497-3597]

\section{Recently Published}

Steiner, R., and Stahley, S., MAP Voltage Transfer Between 10-V Josephson Array Systems, Proceedings of the 1991 National Conference of Standards Laboratories Metrology: A Worldwide Language, Albuquerque, New Mexico, August 18-22, 1991, pp. 205-209.

A Measurement Assurance Program (MAP) for voltage transfer at the $10-\mathrm{V}$ level was performed among six U.S. laboratories currently operating $10-\mathrm{V}$ Josephson array systems. A commercial voltage standard based on four Zener references was used as the transfer device. This experiment provided data on the precision and traceable accuracy of the various array systems relative to the national Si-volt representation at the National Institute of Standards and Technology (NIST), as well as on calibrations involving the new multi-Zener reference standards. Preliminary measurements from five other laboratories show that all agree with NIST to within 0.035 parts per million with a maximum random uncertainty of 0.015 parts per million $(1 \sigma)$.

[Contact: Richard Steiner, (301) 975-4226]

Van Degrift, C.T., Cage, M.E., and Girvin, S.M., Editors, The Integral and Fractional Quantum Hall Effects, Resource Letter QHE-1 (American Association of Physics Teachers, College Park, Maryland 1991), pp. 1-15.
This Resource Letter provides a guide to the literature on the integral and fractional quantum Hall effects. The letter $\mathrm{E}$ after an item indicates elementary level or material of general interest to persons becoming informed in the field. The letter I, for intermediate level, indicates material of somewhat more specialized nature; and the letter A indicates rather specialized or advanced material. An asterisk $\left({ }^{*}\right)$ indicates articles that are especially useful or interesting; a double asterisk $\left({ }^{* *}\right)$ indicates those articles to be included in an accompanying reprint book.

[Contact: Craig T. Van Degrift, (301) 975-4249]

\section{SEMICONDUCTOR MICROELECTRONICS}

\section{$\underline{\text { Silicon Materials }}$}

\section{Released for Publication}

McKeown, D.A., Intermediate-Range-Order Around Copper in Crystalline and Amorphous Silicates.

Recent findings concerning the ordering of networkmodifying calcium environments in silicate glass have attracted scientific interest to the problem of determining the atomic structure of amorphous silicates. From X-ray absorption spectroscopy, ordering of network- modifying copper environments is found for an amorphous copper-silicate, chrysocolla, relative to a crystalline copper-silicate of similar composition, dioptase. In this case, the ordering is due to the copper-copper environment in chrysocolla having a narrower distribution of larger distances, and a larger coordination number than the corresponding coppercopper environment in dioptase. The proposed structural adjustment from dioptase to chrysocolla is simply fewer copper atoms per unit volume of the interstices within the silicate tetrahedral network or between network units.

[Contact: David A. McKeown, (301) 975-3095]

Seraphin, S., Krause, S.J., Roitman, P., Simons, D.S., and Cordts, B.F., Effect of Annealing Ambient on the Removal of Oxide Precipitates in High-Dose Oxygen-Implanted Silicon.

The effect of annealing ambient on the precipitate removal processes in high-dose oxygen-implanted 
silicon (SIMOX) has been studied with transmission electron microscopy, electron energy loss spectroscopy, and secondary ion mass spectroscopy. The rate of removal of oxide precipitates from the top silicon layer in SIMOX is higher during annealing in argon than in nitrogen. The removal is reduced in nitrogen due to the formation of an oxynitride complex at the precipitate surfaces which inhibits oxygen diffusion across the interfaces. Similar effects have been observed for oxide precipitation during nitrogen ambient annealing in bulk silicon.

[Contact: Peter Roitman, (301) 975-2077]

\section{Recently Published}

Campisi, G.J., Roitman, P., and Shontz, G.J., The Role of Annealing Conditions on the Radiation Response of Backgate MOSFETs, Extended Abstract, Proceedings of the Third Workshop on Radiation-Induced and/or Process-Related Electrically Active Defects in Semincoductor-Insulator Systems, Research Triangle Park, North Carolina, September 10-13, 1991, pp. 171-172 (1991).

SIMOX (Separation by IMplantation of OXygen) is the principal SOI technology used for operation in a radiation environment. The suitability of SIMOX in the submicrometer regime on fully depleted devices depends on the radiation response of the dielectric-the beam synthesized buried oxide. In this study, SOI p-MOSFETs were fabricated on the SIMOX substrates annealed at various times and temperatures. The radiation-induced threshold shifts, $\Delta V_{b g}$, of the gate was determined as a function of substrate processing. Preliminary capacitor studies showed a reduction in the radiation-induced flat-band voltage by $40 \%$ when the anneal temperature was increased from 1275 to $1350{ }^{\circ} \mathrm{C}$. The role of anneal temperature on the device properties and radiation response of the buried oxide was investigated in this study. We have correlated the microstructure at the SIMOX interface to the radiation response of the MOSFETs.

[Contact: Peter Roitman, (301) 975-2077]

Campisi, G.J., Roitman, P., Simons, D., and Krull, W.A., A SIMS Study of the Deuterium Distribution in SIMOX Buried Oxides, Extended Abstract, Proceedings of the 1991 IEEE International SOI Conference, Vail Valley, Colorado, October 1-3, 1991, pp. 16-17 (1991).
Hydrogen in $\mathrm{SiO}_{2}$ has been extensively studied and identified as a source of MOS device degradation during hot electron stressing or exposure to ionizing radiation. In this paper, we have used SIMS analysis of deuterium-annealed SIMOX samples to investigate the interaction of hydrogen with the buried oxide.

[Contact: Peter Roitman, (301) 975-2077]

Chi, P.H., Simons, D.S., Roitman, P., and Hughes, H., Quantitative Depth Profiling of Aluminum-Implanted SIMOX by Use of Secondary Ion Mass Spectrometry, Microbeam Analysis-1991, D.G. Howitt, ed., pp. 347-349 (1991).

Separation-by-implanted-oxygen (SIMOX) material has increasingly played an important role in siliconon-insulator technology because of its radiationhardening performance and the high-speed and lowpower usage of devices made on it. However, the performance of this material can be hampered by the presence, even at low levels, of certain impurities. In this paper, we present the results of secondary ion mass spectrometry by use of the matrix ion species ratio method to quantify $\mathrm{Al}$ levels in $\mathrm{Al}$-implanted SIMOX samples.

[Contact: Peter Roitman, (301) 975-2077]

Conley, J.F., Lenahan, P.M., and Roitman, P., Electron Spin Resonance Study of E' Trapping Centers in SIMOX Buried Oxides, IEEE Transactions on Nuclear Science, Vol. 38, No. 6, pp. 1247 1252 (December 1991).

We combine electron spin resonance and capacitance versus voltage measurements with vacuum ultraviolet and ultraviolet illumination sequences to study $\mathrm{E}^{\prime}$ centers in a variety of SIMOX buried oxides. The oxides had all been annealed above $1300{ }^{\circ} \mathrm{C}$. Our results clearly show that $\mathrm{E}^{\prime}$ centers play an important, probably dominating, role in the trapping behavior of these oxides. This role is considerably different from the role that $\mathrm{E}^{\prime}$ centers play in thermal oxides.

[Contact: Peter Roitman, (301) 975-2077]

Conley, J.F., Lenahan, P.M., and Roitman, P., ESR Study of $E^{\prime}$ Trapping Centers in SIMOX Oxides, Extended Abstract, Proceedings of the 1991 IEEE International SOI Conference, Vail Valley, Colorado, October 1-3, 1991, pp. 12-13 (1991).

We explore $E^{\prime}$ trapping centers in separation by 
implanted oxygen (SIMOX) buried oxides with electron spin resonance (ESR) and capacitance vs. voltage (CV) measurements. Through the use of vacuum-ultraviolet and ultraviolet illumination combined with ESR and CV measurements, we present evidence that $E^{\prime}$ centers are important in SIMOX trapping, and that thermal oxide trapping and SIMOX trapping involve different mechanisms.

[Contact: Peter Roitman, (301) 975-2077]

Cortesi, E., El-Ghor, M.K., Hosack, H.H., Allen, L.P., Roitman, P., and Krause, S.J., Evaluation of Secco Etch Technique for Determination of Dislocation Densities in SIMOX Wafers, Extended Abstract, Proceedings of the 1991 IEEE International SOI Conference, Vail Valley, Colorado, October 1-3, 1991, pp. 118-119 (1991).

The greatly improved quality of Separation by IMplantation of OXygen (SIMOX) material now being routinely produced has made the measurement of dislocation densities by plan-view transmission electron microscopy extremely impractical because of the large areas that must be studied. We report here on an extensive study of a Secco etch process for determining dislocation densities that was performed by three different groups using nine SIMOX wafers from the same lot.

[Contact: Peter Roitman, (301) 975-2077]

Krause, S.J., Lee, J.D., Chen, B.L., Seraphin, S., Cordts, B., and Roitman, P., Oxygen Bubble Formation and Evolution During Oxygen Implantation and Annealing of Silicon-on-Insulator Material, Extended Abstract, Proceedings of the 49th Annual Meeting of the Electron Microscopy Society of America, San Diego, California, August 1991, unpaged (1991).

Silicon-on-insulator material fabricated by high-dose oxygen implantation is a material increasingly used for higher speed and radiation-hard circuits. During implantation, a variety of structural changes occur, including the formation of defects, bubbles, precipitates, and the buried oxide layer. The topic of bubble formation and evolution has received only limited study. Sjoreen et al. first reported the presence of spherical, randomly distributed precipitates near the top surface of the silicon layer. El-Ghor et al. further examined these precipitates and proposed that they were cavities filled with oxygen. Maszara confirmed the presence of spheroids filled with oxygen in the silicon top surface region in the $1 \mathrm{~mA} \mathrm{~cm}^{-2}$ as-implanted samples. In this work, transmission electron microscopy techniques were used to investigate the effect of implantation conditions on the bubble formation and the effect of subsequent annealing conditions on the evolution of bubbles.

[Contact: Peter Roitman, (301) 975-2077]

Park, J.C., Krause, S.J., and Roitman, P., Microstructural Changes in Oxygen Implanted SOI Material at Intermediate Annealing Steps in Thermal Ramping, Extended Abstract, Proceedings of the 1991 IEEE International SOI Conference, Vail Valley, Colorado, October 1-3, 1991, pp. 116-117 (1991).

The final microstructure of silicon-on-insulator material fabricated by oxygen implantation (SIMOX) is dependent on the sum of all of the processing steps used to produce the wafer. There have been many reports on microstructures after implantation or annealing, but there is only limited information on microstructural changes occurring during the intermediate stages of processing, in particular, during the thermal ramping cycle. In this work, we are reporting on the microstructural changes in HT SIMOX at various stages in the ramping process by simulating the thermal treatment with 2 -h anneals at intermediate temperatures.

[Contact: Peter Roitman, (301) 975-2077]

Thurber, W.R., Lowney, J.R., Larrabee, R.D., and Ehrstein, J.R., AC Impedance Method for HighResistivity Measurements of Silicon, Journal of the Electrochemical Society, Vol. 138, No. 10, pp. 30813085 (October 1991).

An ac impedance method for measuring the average bulk resistivity of ingots and slices of high-resistivity silicon is presented. Easily removable contacts, such as silver paint, are applied to the end faces of the sample, and the complex impedance of the resulting capacitive sandwich is measured as a function of frequency. The resistivity can be calculated from the frequency of the negative peak in the imaginary part of the impedance and from the absolute values of the real and imaginary parts at that frequency. The spectroscopic nature of the method gives an inherent separation of contact, surface region, and bulk effects as the respective responses usually occur at widely different frequencies. In addition to its intended 
application for measuring bulk resistivity, the method is useful for determining the quality of applied contacts and the effect of surface treatments which result in a significant depletion layer. Plots and the required data can be obtained very quickly with an appropriate microprocessor-based impedance analyzer. Extensive measurements of high-resistivity silicon were done to compare the method with the dc resistance, van der Pauw, and four-probe techniques. The agreement was within $5 \%$ for slices and ingot sections greater than $0.1 \mathrm{~cm}$ in length and resistivity above $5 \mathrm{k} \Omega \cdot \mathrm{cm}$.

[Contact: W. Robert Thurber, (301) 975-2067]

\section{Compound Materials}

Released for Publication

Griffin, J.A., Spencer, M.G., Myers, L.O., Mahmood, M., Woodall, J., Pellegrino, J.G., Comas., J., Glaser, E., and Kennedy, T.A., Interstitial-Substitutional Incorporation of Beryllium in GaAs and $\mathrm{Al}_{\mathrm{x}} \mathrm{Ga}_{1-\mathrm{x}}$ As.

At low doping levels there is a linear relationship between the Be flux and the net acceptor concentration in $\mathrm{GaAs}$ and $\mathrm{Al}_{\mathrm{x}} \mathrm{Ga}_{1-\mathrm{x}}$ As layers grown by molecular beam epitaxy. However, at high fluxes the acceptor concentration saturates. We show that this effect is due to the increased interstitial incorporation of the Be atom which is controlled by the Fermi-level position in the crystal during growth. In this paper we investigate the $\mathrm{Be}$ incorporation dependence on As pressure, $\mathrm{Al}$ mole function, and growth temperature. Also, we present evidence for the observation of the Be interstitial by optically detected magnetic resonance.

[Contact: Joseph G. Pellegrino, (301) 975-2123]

Kopanski, J.J., Lowney, J.R., Novotny, D.B., Seiler, D.G., Simmons, A., and Ramsey, J., HighSpatial-Resolution Resistivity Mapping Applied to Mercury Cadmium Telluride, to be published in the Proceedings of the 1991 U.S. Workshop on the Physics and Chemistry of Mercury Cadmium Telluride and Other II-VI Compounds, Dallas, Texas, October 8-10, 1991. [Proceedings to be published in a special issue of the Journal of Vacuum Science and Technology B.]

The fine-scale resistivity va riations of bulk slices of $n$ type $\mathrm{Hg}_{1-\mathrm{x}} \mathrm{Cd}_{\mathrm{x}} \mathrm{Te}$ grown by the solid-state recrys- tallization (SSR) process and $\mathrm{HgCdTe}$ grown by liquid phase epitaxy (LPE) on SSR substrates were mapped at room temperature $(297 \mathrm{~K})$. An automatic probe station was used to make four-probe resistance measurements, spaced $80 \mu \mathrm{m}$ apart, on lithographically defined metal-to-HgCdTe contacts. Most slices of SSR $\mathrm{HgCdTe}$ were found to have resistivity that increased from the center to the outside edge, and some SSR material also showed small inclusions with different resistivity than the surrounding material. LPE material was found to have a more random variation in resistivity than SSR $\mathrm{HgCdTe}$. Also, the metal semiconductor contact resistivity of $\mathrm{Ni} / \mathrm{InPb} / \mathrm{In}$ contacts to n-type $\mathrm{HgCdTe}$ was measured to be in the range of 0.3 to $20 \times 10^{-5} \Omega-\mathrm{cm}^{2}$ for $\mathrm{HgCdTe}$ resistivities varying from 0.01 to $0.1 \Omega$-cm.

[Contact: Joseph J. Kopanski, (301) 975-2089]

Vanzura, E.J., Weil, C.M., and Williams, D.F.; Complex Permittivity Measurements of GalliumArsenide Using a High-Precision Resonant Cavity, to be published in the Conference Record, Conference on Precision Electromagnetic Measurements (CPEM '92), Paris, France, June 9-12, 1992.

Data are presented on the complex permittivity of gallium arsenide, as measured at room temperature in the 8 - to $12-\mathrm{GHz}$ frequency range. The measurements were performed using a mode-filtered cylindrical cavity resonator with helically wound walls. The estimated accuracies are $\pm 0.4 \%$ in relative permittivity and $\pm 5 \%$ in loss tangent at $10 \mathrm{GHz}$.

[Contact: Eric J. Vanzura, (303) 497-5752]

\section{Recently Published}

Bennett, H.S., Lowney, J.R., Tomizawa, M., and Ishibashi, T., Experimentally Verified Majority and Minority Mobilities in Heavily Doped GaAs for Device Simulations, Extended Abstract, 1991 International Workshop on VLSI Process and Device Modeling (1991 VPAD), Oiso, Japan, May 26-27, 1991, pp. 114-115. [Complete paper will appear in a Special Issue on Selected Papers from '91 VPAD.]

Low-field mobilities and velocity-versus-electric-field relations are among the key input parameters for drift-diffusion simulations of field-effect and bipolar transistors. For example, most device simulations that treat scattering from ionized impurities contain mobilities or velocity-versus-field relations based on 
the Born approximation (BA). The BA is insensitive to the sign of the charged impurity and is especially poor for ionized impurity scattering because of the relatively strong scattering of long-wavelength carriers, which have low energies, and therefore violate the validity condition for the BA. Such carriers occur at high symmetry points in the Brillouin zone and are critical for device behavior.

[Contact: Herbert S. Bennett, (301) 975-2047]

Dagata, J.A., Tseng, W., Bennett, J., Schneir, J., and Harary, H.H., $\mathbf{P}_{\mathbf{2}} \mathbf{S}_{\mathbf{5}}$ Passivation of GaAs Surfaces for Scanning Tunneling Microscopy in Air, Applied Physics Letters, Vol. 59, No. 25, pp. 32883290 (16 December 1991).

We report a novel method of $\mathrm{GaAs}$ substrate preparation which imparts significantly improved topographical and chemical uniformity to the surface. The procedure, employing an aqueous $\mathrm{P}_{2} \mathrm{~S}_{5} /\left(\mathrm{NH}_{4}\right)_{2} \mathrm{~S}$ solution, leaves the surface in a highly ordered state and resistant to air oxidation for periods of a day or more without the presence of foreign chemical layer such as sulfur. Surface quality was determined by scanning tunneling microscopy (STM), time-of-flight secondary ion mass spectrometry, reflection highenergy electron diffraction, and X-ray photoelectron spectroscopy. The remarkable stability and smoothness of treated III-V surfaces is illustrated by STM imaging of an $\mathrm{Al}_{0.51} \mathrm{Ga}_{0.49} \mathrm{As} / \mathrm{GaAs}$ superlattice in air. The superlattice consisted of periodic alternating $\mathrm{AlGaAs} / \mathrm{GaAs}$ layers of various thicknesses from 10 to $1000 \mathrm{~nm}$.

[Contact: Wen F. Tseng, (301) 975-5291]

Doss, M.G., Chandler-Horowitz, D., Marchiando, J.F., Krause, S., and Seraphin, S., Analysis for the Characterization of Oxygen Implanted Silicon (SIMOX) by Spectroscopic Ellipsometry, Proceedings of the Materials Research Society Symposium, Boston, Massachusetts, November 26-December 1, 1990, Vol. 209, pp. 493-498 (1991).

Samples of SIMOX have been prepared using a highcurrent implanter (density $\approx 1 \mathrm{~mA} / \mathrm{cm}^{2}$ ) and annealed at $1300{ }^{\circ} \mathrm{C}$ for $6 \mathrm{~h}$. Conventional and high-resolution transmission electron microscopy reveal unusual structure in these samples. Spectroscopic ellipsometry has been used to analyze these structures. Ellipsometric measurements were collected at an angle of incidence of $75.5 \mathrm{deg}$, with energies from 1.5 to $5.0 \mathrm{eV}$, and using a rotating polarizer configuration. The measurements were analyzed with three models: a threelayer model, a four-layer model, and a five-layer model. The five-layer model provided the best fit of the three. This model identified a layer of crystalline $\mathrm{Si}$ inclusions ("islands") within the $\mathrm{SiO}_{2}$ layer. A method is presented that provides initial estimates for the thickness of the top three layers to help start the regression analysis.

[Contact: Deane Chandler-Horowitz, (301) 975-2084]

Huang, D., Kallergi, M., Aubel, J., Sundaram, S., DeSalvo, G.C., and Comas, J., Lattice Damage and Atomic Mixing Induced by $\mathrm{As}^{++}$Implantation and Thermal Annealing in AlAs/GaAs Multiple Quan. tum-Well Structures, Journal of Applied Physics, Vol. 70, No. 8, pp. 4181-4189 (15 October 1991).

The lattice damage and the nature of the atomic intermixing of $\mathrm{Al}$ and $\mathrm{Ga}$ induced by $\mathrm{As}^{++}$implantation and thermal annealing in $\mathrm{AlAs} / \mathrm{GaAs}$ multiple quantum-well structures were investigated. The photoluminescence spectra, which show multiple peaks after implantation and annealing, were analyzed based on the shifts of the excitonic peaks arising from quantum wells located at different depths. The depth profiles of intermixing were obtained using a procedure of successive layer-by-layer chemical etching following photoluminescence measurements. It is found that the mixing is maximum near the sample surface and decreases monotonically with depth, suggesting that the profiles follow the ion-induced damage more closely than the ion density. It is also observed that the damage extends beyond $1 \mu \mathrm{m}$. Within $0.3 \mu \mathrm{m}$ from the surface, the damage is heavy and the atomic intermixing increases rapidly with ion dose, indicating the damage is structural. Beyond 0.3 $\mu \mathrm{m}$, the degree of intermixing is only sensitive to the anneal temperature but not to the implantation dose. The results show that both direct collisions and interdiffusion are responsible for the atomic mixing. For the samples implanted with ion doses below $10^{14}$ $1 / \mathrm{cm}^{-2}$ and annealed at $650^{\circ} \mathrm{C}$, the optical activation from radiation damage is appreciable. However, the interdiffusion becomes important at temperatures near and above $800^{\circ} \mathrm{C}$.

[Contact: James Comas, (301) 975-2061]

Littler, C.L., Loloee, M.R., Zawadzki, W., and Seiler, D.G., Bound Hole Excitations in p$\mathbf{H g}_{\mathbf{0 . 7 6}} \mathbf{C d}_{\mathbf{0 . 2 4}} \mathrm{Te}$, Proceedings of the 20th Interna- 
tional Conference on the Physics of Semiconductors, Thessaloniki, Greece, August 6-10, 1990, Vol. 3, pp. 2263-226 (1990).

Bound-hole transitions originating from a deep level to light-hole Landau levels have been observed for the first time in $\mathrm{HgCdTe}$. Resonances have been seen in the photovoltaic response of a p-type $\mathrm{Hg}_{0.76} \mathrm{Cd}_{0.24} \mathrm{Tc}$ sample subjected to $\mathrm{CO}_{2}$ laser radiation. The transitions are well described by the Pidgeon-Brown energy band model, yielding an activation energy of $32 \pm 2$ $\mathrm{meV}$ above the valence band edge for the deep level. [Contact: David G. Seiler, (301) 975-2081]

Littler, C.L., Yoon, I.T., Song, X.N., Zawadzki, W., Pfeffer, P., and Seiler, D.G., Orbital and Spin Anisotropy of Conduction Electrons in InSb, Proceedings of the 20th International Conference on the Physics of Semiconductors, Thessaloniki, Greece, August 6-10, 1990, Vol. 3, pp. 1763-1766 (1990).

The anisotropy of the orbital and spin properties of conduction electrons in InSb has been measured simultaneously for the first time using a cyclotronresonance-type experiment. A novel approach was used to measure precisely small shifts of the resonant field positions with respect to the crystal axes -- the cyclotron resonance signals were detected at the same time from two differently oriented samples. The data have been described using a five-level $k \cdot p$ energy band model, which accounts for both the nonparabolicity and anisotropy of the conduction band in III-V compounds in the presence of a magnetic field.

[Contact: David G. Seiler, (301) 975-2081]

Pellegrino, J., Griffin, J., Myers, L., and Spencer, M., Beryllium Doping in MBE-Grown GaAs and AlGaAs, Proceedings of the Materials Research Society Symposium, Boston, Massachusetts, November 27-December 2, 1989, Vol. 163, pp. 881-886 (1990).

Beryllium is an effective p-dopant in GaAs and $\mathrm{AlGaAs}$ and plays an important role in device characterizations of heterobipolar transistors. This work addresses the doping and mobility properties for two series of beryllium-doped samples: GaAs and AlGaAs. Within each series the doping ranged between levels of $3 \times 10^{15} \mathrm{~cm}^{-3}$ to $5 \times 10^{19} \mathrm{~cm}^{-3}$. Mobility and carrier concentrations were obtained through Hall and Polaron measurements. The doping concentration results suggest the onset of carrier compensation at higher doping levels. One possible explanation is that for high doping levels, Be is incorporated as interstitial donors. A thermodynamic model is used to explain the observations.

[Contact: Joseph G. Pellegrino, (301) 975-2123]

\section{Device Physics and Modeling}

\section{Released for Publication}

Albers, J., Semiconductor Measurement Technology: A Collection of Computer Programs for Two-Probe Resistance (Spreading Resistance) and Four-Probe Resistance Calculations: RESPAC, to be published as NIST Special Publication 400-91.

This report presents and describes a number of FORTRAN programs which may be used to perform two-probe resistance (spreading resistance) and fourprobe resistance calculations for vertically nonuniform resistivity structures. These programs fall into three general categories. They are: 1) programs for calculating the two-probe resistance (spreading resistance) from the resistivity profile, 2 ) programs for calculating the resistivity profile from the two-probe resistance (the inverse of 1), and 3 ) programs for calculating the four-probe resistance from the resistivity profile. Programs in the first and third category are useful for understanding the effects of resistivity variations on the two-probe resistance (spreading resistance) and the four-probe resistance. Programs in the second category are useful for extracting the resistivity profile from spreading resistance data (either measured or calculated). All of the programs are based upon the Schumann and Gardner solution of the multilayer Laplace equation. As such, local charge neutrality is assumed. The limitations of this assumption are described in the text. The first part of this report consists of an outline of the derivation of the Schumann and Gardner multilayer Laplace equations. In addition, there is a discussion of the evolution and simplification of this model which has taken place over the past two decades. This part of the report is intended to provide the reader with a background so as to make optimal use of the computer programs. The second part of the report contains a discussion of the structure and inner workings of each of the programs. Special attention is paid to the aspects which make the individual programs different from others in the same category. In addition, sample 
input data used in the programs and the corresponding output data calculated by the programs are also presented. The final parts of the report (the appendices) contain annotated, internally documented listings of the FORTRAN source codes. In all, there are ten programs contained in the RESPAC package. The FORTRAN source code (total of about 123 kbytes) and sample input and output data files are available in ASCII format using a number of transfer vehicles. These include: standard 8-track magnetic tape $($ ASCII, density $=1600$, record $=80$, block $=1600)$, 5.25-in. (360 kbyte and 1.2 Mbyte) DOS-formatted floppy disks, and using electronic mail over the Internet. This package is self-contained and is straightforward to run once the FORTRAN is compiled and linked by the user-supplied software. The sample input and output data files are included so that the user can check the programs for proper operation as well as to become acquainted with the setup and use of the codes.

[Contact: John Albers, (301) 975-2075]

Bennett, H.S., and Lowney, J.R., Calculated Majorityand Minority-Carrier Mobilities in Heavily Doped Silicon and Comparisons with Experiment.

As silicon devices approach $0.1 \mu \mathrm{m}$ in size, it will be essential to have accurate values for the majority and minority mobilities of electrons and holes. These mobilities have been calculated in silicon for donor and acceptor densities between $10^{17} \mathrm{~cm}^{-3}$ and $10^{20}$ $\mathrm{cm}^{-3}$. All the important scattering mechanisms have been included. The ionized impurity scattering has been treated with a quantum-mechanical phase-shift analysis. The results are in good agreement with experiment, but predict that the change of minority electron mobility with increasing dopant density should decrease slightly at high dopant densities for a small range of densities. This effect occurs mainly because of the reduction of plasmon scattering. Some recent experiments support these findings. In addition, the ionized impurity scattering rates calculated from the quantum-mechanical phase shifts and those rates calculated from the Born approximation are shown to differ by more than factors of three. The Born approximation is not valid for low energy carriers near band extrema. Carrier scattering rates are key input quantities for Monte Carlo calculations of carrier-velocity versus electric-field relations and of simulations of device behavior. Our calculations do not treat the density-of-states modifications due to heavy doping, which should have only a small effect on the mobility at room temperature.

[Contact: Herbert S. Bennett, (301) 975-2047]

Geist, J., Chandler-Horowitz, D., Robinson, A.M., James, C.R., Kohler, R., and Goebel, R., Numerical Modeling of Silicon Photodiodes for High-Accuracy Applications, to be published as NISTIR 4592.

The purpose of this National Institute of Standards and Technology Interagency/Internal Report (NISTIR) is to provide the source code for two Turbo Pascal 5.5 programs and an MSDOS batch program, along with a paper that describes the programs and provides examples of their use. These three programs serve as a batch mode interface to support highaccuracy photodiode modeling with Version 2 of the semiconductor device modeling program optimized for solar cell modeling, and it is somewhat difficult to access the highest accuracy available from PC-1D through this interface. Part I describes PC-1D from the point of view of high-accuracy photodiode modeling and describes the programs that support its use in this application. Parts II and III present examples of the use of the programs described in Part I to model two different types of experiments performed on silicon photodiodes in various high-accuracy applications.

[Contact: Jon Geist, (301) 975-2066]

\section{Recently Published}

Bennett, H.S., Lowney, J.R., Tomizawa, M., and Ishibashi, T., Experimentally Verified Majority and Minority Mobilities in Heavily Doped GaAs for Device Simulations, Extended Abstract, 1991 International Workshop on VLSI Process and Device Modeling (1991 VPAD), Oiso, Japan, May 26-27, 1991, pp. 114-115. [Complete paper will appear in a Special Issue on Selected Papers from '91 VPAD.]

Low-field mobilities and velocity-versus-electric-field relations are among the key input parameters for drift-diffusion simulations of field-effect and bipolar transistors. For example, most device simulations that treat scattering from ionized impurities contain mobilities or velocity-versus-field relations based on the Born approximation (BA). The BA is insensitive to the sign of the charged impurity and is especially poor for ionized impurity scattering because of the relatively strong scattering of long-wavelength carriers, 
which have low energies, and therefore violate the validity condition for the BA. Such carriers occur at high symmetry points in the Brillouin zone and are critical for device behavior.

[Contact: Herbert S. Bennett, (301) 975-2047]

Lowney, J.R., and Mayo, S., Analysis of Persistent Photoconductivity Due to Potential Barriers, Proceedings of the Third Workshop on RadiationInduced and/or Process-Related Electrically Active Defects in Semiconductor-Insulator Systems, Research Triangle Park, North Carolina, September 1013, 1991, pp. 95-102.

Persistent photoconductivity has been seen in thin silicon resistors fabricated with SIMOX material at temperatures between 60 and $220 \mathrm{~K}$. This effect has been attributed to the depletion of carriers near the interface between the top silicon layer and the buried oxide, which is due to the large number of surface traps at this interface. The depletion of carriers is accompanied by a built-in field on the order of 10,000 $\mathrm{V} / \mathrm{cm}$, which causes a potential barrier that is about a quarter of the energy gap of silicon. The theory of the recombination kinetics of majority carriers with minority carriers trapped at the interface on the other side of a potential barrier is studied. Both the possibilities of tunneling and thermal activation have been considered. The results show that thermal activation dominates at the temperatures of the NIST measurements in SIMOX material, while at lower temperatures tunneling would dominate.

[Contact: Jeremiah R. Lowney, (301) 975-2048]

Mitter, C.S., Hefner, A.R., Jr., Chen, D.Y., and Lee, F.C., Insulated Gate Bipolar Transistor (IGBT) Modeling Using IG-Spice, Proceedings of the 1991 IEEE Industry Applications Society Meeting, Dearborn, Michigan, September 28-October 4, 1991, pp. 1515-1521.

A physics-based model for the Insulated Gate Bipolar Transistor (IGBT) is implemented into the widely available circuit simulation package IG-Spice. Based on analytical equations describing the semiconductor physics, the model accurately describes the nonlinear junction capacitances, moving boundaries, recombination, and carrier scattering, and effectively predicts the device conductivity modulation. In this paper, the procedure used to incorporate the model into IGSpice and various methods necessary to ensure convergence are described. The effectiveness of the Spice-based IGBT model is demonstrated by investigating the static and dynamic current sharing of paralleled IGBTs with different device model parameters. The simulated results are verified by comparison with experimental results.

[Contact: Allen R. Hefner, Jr., (301) 975-2071]

Tomizawa, M., Ishibashi, T., Bennett, H.S., and Lowney, J.R., Verification of Effective Intrinsic Carrier Concentrations for Numerical Simulations of Gallium Arsenide Bipolar Transistors, Extended Abstract, Proceedings of the 1991 International Workshop on VLSI Process and Device Modeling (1991 VPAD), Oiso, Japan, May 26-27, 1991, pp. 116-117.

Using the best available physical models is essential for predictive numerical simulations of heterostructure GaAs bipolar transistors. Recent theoretical calculations of $n_{\mathrm{ie}}$ for $\mathrm{GaAs}$ at $300 \mathrm{~K}$ have been verified experimentally for the first time by measurements on devices.

[Contact: Herbert S. Bennett, (301) 975-2047]

\section{Insulators and Interfaces}

[For Separation by Implanted Oxygen (SIMOX) and for Silicon-on-Insulator (SOI) see Silicon Materials]

\section{Recently Published}

Kim, K.H., Bell, M.I., Dozier, C.M., Freitag, R.K., and Bouldin, C.E., Correcting for X-Ray Energy Calibration Error Caused by Misalignment of a Right-Angle Linkage Monochromator [original title: Correcting for Error Caused by Misalignment of Right-Angle Linkage Monochromator], Review of Scientific Instrumentation, Vol. 62, No. 4, pp. 982985 (April 1991).

Small alignment errors of right-angle linkage monochromators typical to many XAFS beamlines can cause significant errors in the energy calibrations. A 1-deg misalignment produces errors greater than 1 $\mathrm{keV}$ over the hard $\mathrm{X}$-ray operating range of a typical monochromator. The energy error caused by such misalignments is analyzed and its mathematical form given. The error can be corrected by inverting the expression, and the amount of misalignment determined by accurate energy measurements at a few points. The accuracy of the corrections is tested. The 
effects of this error on X-ray absorption fine structure data and their interpretation are also discussed. [Contact: David G. Seiler, (301) 975-2081]

Mayo, S., Lowney, J.R., and Roitman, P., Measurement of Interface Defects in Gated SIMOX Structures, Extended Abstract, Proceedings of the 1991 IEEE International SOI Conference, Vail Valley, Colorado, October 1-3, 1991, pp. 52-53.

Defects in gated or ungated thin film resistors have been characterized by photoinduced transient spectroscopy (PITS). The resistors were fabricated with nor p-type SIMOX (separation by implanted oxygen) wafers implanted with $200-\mathrm{keV}$ oxygen to $1.8 \mathrm{x} 10^{18}$ $\mathrm{cm}^{-2}$ total fluence. One wafer used for gated resistor fabrication was implanted at $595^{\circ} \mathrm{C}$ and sequentially annealed at $1325{ }^{\circ} \mathrm{C}$ for $4 \mathrm{~h}$ in argon (plus $0.5 \%$ oxygen) followed by $4 \mathrm{~h}$ in nitrogen (plus $0.5 \%$ oxygen). Another wafer used for ungated resistor fabrication was implanted at $650{ }^{\circ} \mathrm{C}$ and annealed at $1275^{\circ} \mathrm{C}$ for $2 \mathrm{~h}$ in nitrogen (plus $0.5 \%$ oxygen). PITS data indicate that electron or hole traps in the conductive silicon film are located at the film-buried silica interface. We estimate the average interface trap density in the SIMOX structure to be in the $10^{11} \mathrm{~cm}^{-2}$ range.

[Contact: Santos Mayo, (301) 975-2045]

Richter, M., Woicik, J.C., Pianetta, P., Miyano, K.E., Kendelewicz, T., Bouldin, C.E., Spicer, W.E., and Lindau, I., Surface Extended X-Ray Adsorption Fine Structures Studies of the $\mathrm{Si}(001) 2 \times 1-\mathrm{Sb}$ Interface, Journal of Vacuum Science Technology A, Vol. 9, No. 3, pp. 1951-1955 (May/Jun 1991).

Surface X-ray adsorption fine structure (SEXAFS) has been used to investigate the structure of $\mathrm{Sb}$ on the $\mathrm{Si}(001) 2 \times 1$ surface. The coverage of $\mathrm{Sb}$ which remains after annealing thick layers at $375^{\circ} \mathrm{C}$, previously reported to be one monolayer (ML), is found in this work to form a disordered overlayer with threedimensional $\mathrm{Sb}$ clusters. This finding is concluded from the $\mathrm{Sb} \mathrm{L}_{3}$ absorption spectra which are similar for this coverage to that of bulk Sb. After a $550{ }^{\circ} \mathrm{C}$ anneal, Auger electron spectroscopy and scanning tunneling microscopy (STM) show that about one ML of Sb remains. Phase and amplitude analysis of the $\mathrm{Sb} \mathrm{L}_{3}$ edge SEXAFS shows that the remaining $\mathrm{Sb}$ atoms occupy a modified bridge site with a Si-Sb bond length of $2.63 \pm 0.04 \AA$. The Sb atoms form dimers with a Sb-Sb bond length of $2.91 \pm 0.04 \AA$, which is almost identical to the bulk Sb-Sb bond length of 2.90 $\AA$. The Sb atoms lie $1.74 \pm 0.06 \AA$ above the $\mathrm{Si}(001)$ surface. STM confirms the dimer structure of the Sb overlayer. Low-energy electron diffraction performed on vicinal single-domain $\mathrm{Si}$ show that these dimers form rows that run perpendicular to the $\mathrm{Si}$ dimer rows.

[Contact: David G. Seiler, (301) 975-2431]

Woicik, J.C., Bouldin, C.E., Bell, M.I., Cross, J.O., Tweet, D.J., Swanson, B.D., Zhang, T.M., Sorensen, L.B., King, C.A., Hoyt, J.L., Pianetta, P., and Gibbons, J.F., Conservation of Bond Lengths in Strained Ge-Si Layers, Physical Review B, Vol. 43, No. 3, pp. 2419-2422 (15 February 1991).

The combined techniques of X-ray absorption fine structure (EXAFS) and X-ray diffraction have been used to study the strain and bond distortions in epitaxial GeSi on Si(001). In a $31 \%-G e, 340-\AA$ pseudomorphic GeSi film, the Ge-Ge and Ge-Si firstneighbor bond lengths have been found to be $2.44 \pm$ $0.02 \AA$ and $2.38 \pm 0.02 \AA$, respectively. The lattice parameter perpendicular to the $\mathrm{GeSi} / \mathrm{Si}(001)$ interface has been found to be $a_{\perp}=5.552 \pm 0.002 \AA$, in agreement with the predictions of macroscopic elastic theory. These results show that the bond length strain in the epitaxial layer appears in the second and higher coordination shells, rather than in the nearest neighbor bond lengths which remain the same as in unstrained GeSi. A microscopic model is presented which accounts for these findings.

[Contact: David G. Seiler, (301) 975-2046]

Woicik, J.C., Kendelewicz, T., Miyano, K.E., Bouldin, C.E., Meissner, P.L., Pianetta, P., and Spicer, W.E., Local Bonding Structure of Sb on Si(111) by Surface Extended X-Ray Absorption Fine Structure and Photoemission, Physical Review B, Vol. 43, No. 5, pp. $4331-4339$ (15 February 1991).

The combined techniques of surface extended X-ray absorption fine structure (SEXAFS) and high-resolution core and valence photoelectron spectroscopy have been used to study the local bonding structure of the $\mathrm{Sb} / \mathrm{Si}(111)$ interface. From photoemission, we find that the $\mathrm{Sb}$ atoms absorb in a unique environment which completely saturates the dangling bonds of the Si(111) surface, and which completely eliminates the surface components of the Si 2 p core level spectrum. 
The Sb-induced Si $2 p$ interfacial core level shift has been found to be shifted $0.20 \pm 0.02 \mathrm{eV}$ towards higher binding energy with an intensity which corresponds to $1 \mathrm{ml}$ of surface atoms. The SEXAFS determination of the absolute surface coordination numbers and bond lengths within the first $\mathrm{Sb}$ shell is $2.1 \pm 0.3 \mathrm{Sb}$ atoms at $2.86 \pm 0.02 \AA$ and $2.0 \pm 0.4 \mathrm{Si}$ atoms at $2.66 \pm 0.03 \AA$. Combined, these results indicate that $\mathrm{Sb}$ trimmers occupy the threefold atop sites of the $\mathrm{Si}(111)$ surface where each $\mathrm{Sb}$ atom is bonded to two Si atoms in a modified bridge configuration.

[Contact: David G. Seiler, (301) 975-2081]

Woicik, J.C., Kendelewicz, T., Miyano, K.E., Bouldin, C.E., Meissner, P.L., Pianetta, P., and Spicer, W.E., Structure of the $\operatorname{Si}(111) \sqrt{3} x \sqrt{3}-\mathrm{Sb}$ Interface by Surface X-Ray Absorption Fine Structure and Photoemission, Journal of Vacuum Science Technology A, Vol. 9, No. 3, pp. 1956-1961 (May/Jun 1991). [Proceedings of the American Vacuum Society Meeting, Toronto, Canada, October $8-12,1990$.

The combined techniques of surface extended X-ray absorption fine structure (SEXAFS) and high-resolution core level photoelectron spectroscopy have been used to investigate the local bonding structure of the $\mathrm{Sb} / \mathrm{Si}(111)$ interface. We find that the adsorption of 1 monolayer (ML) of Sb completely eliminates the surface components of the $\mathrm{Si} 2 \mathrm{p}$ core level spectrum. The Sb-induced Si $2 p$ interfacial core level has been found to be shifted $0.20 \pm 0.02 \mathrm{eV}$ towards higher binding energy with an intensity that corresponds to the top $1 \mathrm{ML}$ of surface atoms. The SEXAFS determination of the absolute surface coordination numbers and bond lengths within the first Sb shell is $2.1 \pm$ $0.3 \mathrm{Sb}$ atoms at $2.86 \pm 0.02 \AA$ and $2.0 \pm 0.4 \mathrm{Si}$ atoms at $2.66 \pm 0.03 \AA$. Together, these results indicate that $\mathrm{Sb}$ trimers occupy the threefold atop sites of the $\mathrm{Si}(111)$ surface where each $\mathrm{Sb}$ atom is bonded to two $\mathrm{Si}$ atoms in a modified bridge configuration.

[Contact: David G. Seiler, (301) 975-2081]

\section{Dimensional Metrology}

\section{Released for Publication}

Geist, J., Belzer, B.J., Miller, M.L., and Roitman, P., Optical Calibration of a Submicrometer Magnification Standard.
The calibration of a new submicrometer magnification standard for electron microscopes is described. The new standard is based on the width of a submicrometer thermal-oxide film sandwiched between a silicon single-crystal substrate and a polysilicon capping layer. The calibration is based on an ellipsometric measurement of the oxide thickness before the polysilicon layer is deposited on the oxide. The uncertainty in the derivation of a thickness for the layer from the ellipsometric parameters is also derived.

[Contact: Jon Geist, (301) 975-2066]

\section{Recently Published}

Larrabee, R.D., Linholm, L.W., and Postek, M.T., Submicrometer Critical Dimension Metrology, "Handbook of VLSI Microlithography," Chapter 3, pp. 148-237 (Noyes Publications, Park Ridge, New Jersey, 1991).

The never-ending push of the semiconductor industry toward submicrometer feature sizes on integrated circuits and in discrete devices has led to the situation where the development of the techniques of fabrication of submicrometer features has exceeded the development of the metrological techniques required to accurately measure and characterize these features. At the present time (1987), there are no submicrometer critical-dimension standards available from the National Institute of Standards and Technology (NIST) for feature-size measurements on integrated circuits and thus, no way to achieve traceability to NIST for such measurements. NIST has active programs to develop the techniques for measuring such features and for certifying standards using optical microscopy and scanning electron microscopy. In addition, NIST has an active program to develop electrical dimensional metrology using specially designed test patterns. This chapter summarizes these programs and highlights some of the results obtained to date. This chapter does not discuss some of the potential or proposed techniques of dimensional metrology that might be used in the more distant future (e.g., scanning tunneling microscopy) and does not evaluate any of the proposed nonconventional modes of operation of the optical or scanning-electron microscopes (e.g., near-field optical imaging). The purpose of the chapter is to describe the general state of the art in submicrometer dimensional metrology as presently practiced by the semiconductor industry, to 
highlight some of the problems with such practice, and to give suggestions on how to improve the precision and accuracy of such measurements.

[Contact: Robert D. Larrabee, (301) 975-2298]

Postek, M.T., and Keery, W.J., Cryopump Vibration Isolation System for an SEM, Scanning, Vol. 13, pp. 404-409 (1991).

A standard commercially available cryopump system has been installed on a scanning electron microscope (SEM) using specially optimized vacuum vibration isolation bellows. This installation was successful in reducing the cryopump-induced vibration to a level that did not degrade the standard performance or resolution of the SEM in the pump-on mode and, in the pump-off (or coasting) mode, eliminated all measurable instrument-induced vibration (i.e., from the vacuum system). This paper outlines the manner in which this performance has been accomplished, and presents the results of an experiment demonstrating the reduction of specimen contamination provided by this type of vacuum system.

[Contact: Michael T. Postek, (301) 975-2299]

\section{Integrated-Circuit Test Structures}

\section{Released for Publication}

Cresswell, M.W., Khera, D., Linholm, L.W., and Schuster, C.E., A Directed-Graph Classifier of Semiconductor Wafer-Test Patterns.

This paper describes a technique for training an expert system for semiconductor wafer fabrication process diagnosis. The technique partitions an existing set of electrically tested semiconductor wafers into groups so that all wafers within each group have similar spatial distributions of the electrical test data across the selected die sites. The spatial distribution of test data from the selected die sites on each wafer is referred to as the test pattern of that wafer. The supposition is that test patterns reflect the processing histories of the respective wafers. Furthermore, it is presumed that the processing history of each of the existing partitioned set of wafers is at least partially known. A directed graph that is developed by the partitioning algorithm is then able to efficiently classify a new incoming wafer to one of the groups established during partitioning. The directed graph identifies the incoming wafer's processing history on the basis of its test pattern. The processing history thus determined is a first indication of the wafer's process diagnosis. In addition, the way in which similar processing histories agglomerate within particular groups provides a facility for formulating rules for a knowledge-based diagnostic expert system. Intralevel isolation test structure data are used to illustrate the principles of the construction of the directed graph. The technique is appropriate for any available test pattern, whether it is extracted from a test structure or from a functional integrated circuit device or from both.

[Contact: Michael W. Cresswell, (301) 975-2072]

\section{Recently Published}

Schuster, C.E., Linholm, L.W., and Gillespie, J.K., High-Density Test Structures for Assessing Microwave/Millimeter Wave Monolithic Integrated Circuit (MIMIC) Performance, Digest of Papers, Proceedings of the 1991 Government Applications Conference(GOMAC), Orlando, Florida, November 5-7, 1991, Vol. XVII, pp. 335-338.

This paper discusses the unique, high-density implementation of microelectronic test structures used to diagnose and predict MIMIC performance under MIMIC Phase 1, Task 4.E. It also presents assessments and recommendations, based on Task 4.E data, to improve and extend current parametric test structure methods for use in future efforts to correlate and monitor MIMIC material, process, and device characteristics.

[Contact: Constance E. Schuster, (301) 975-2241]

Troccolo, P., Mantalas, L., Allen, R.A., and Linholm, L.W., Extending Electrical Measurements to the $0.5 \mu \mathrm{m}$ Regime, Proceedings of SPIE (The International Society for Optical Engineering, P.O. Box 10, Bellingham, Washington 98227-0010), Integrated Circuit Metrology, Inspection, and Process Control V, Vol. 1464, pp. 90-103 (1991).

The purpose of this work was to extend the design criteria of electrical test structures to the half-micrometer linewidth region. At $0.5 \mu \mathrm{m}$, process limitations place constraints on the functionality and usefulness of electrical test structures based on conventional design criteria. In particular, small total variations from lens aberrations/distortions and proximity/corner rounding effects in the patterning of 
the smallest lines achievable (less than $0.5 \mu \mathrm{m}$ ) can result in structure failure. This was particularly significant when orthogonal voltage taps at minimum design geometries were used. As geometries decrease in size and control over the process and equipment tightens, the intrinsic error in conventional structures has increased as a percentage of the total measurement. The design criteria of these structures have been further modified and improved in order to address known lithographic limitations and establish a more process-tolerant design. The resulting measurement precision accommodating these changes is discussed to provide the framework for achieving the highest practical performance attainable from both the test structure and the measurement system.

[Contact: Richard A. Allen, (301) 975-5026]

Microfabrication Technology

Released for Publication

Marshall, J.C., Parameswaran, M., Zaghloul, M., and Gaitan, M., Methodology for the ComputerAided Design of Silicon-Micromachined Devices in a Standard CMOS Process.

The methodology for implementing the design of silicon-micromachined devices in a standard CMOS foundry process is discussed, and a modified Magic technology file is introduced. The modified technology file is used to design silicon-micromachined devices that are fabricated using a standard CMOS foundry through the MOSIS service. An additional maskless etch in EDP is required to realize the micromechanical structures once devices are delivered. The modified technology file implements a layer that we call "open" that consists of a combination of vias and contact cuts. This open area exposes the silicon surface for an anisotropic etch procedure that creates suspended bridges of polysilicon or metal encapsulated in $\mathrm{SiO}_{2}$. Results from fabricated chips are included.

[Contact: Janet C. Marshall, (301) 975-2049]

Recently Published

Dagata, J.A., Tseng, W., Bennett, J., Schneir, J., and Harary, H.H., Nanolithography on III-V Semiconductor Surfaces Using a Scanning Tunneling Microscope Operating in Air, Journal of Applied
Physics, Vol. 70, No. 7, pp. 3661-3665 (1 October 1991).

Nanometer-scale pattern generation on III-Vsemiconductor substrates using a scanning tunneling microscope (STM) operating in air is demonstrated. The sample substrates, consisting of arsenic-capped, epitaxial layers of $n$-doped $\mathrm{GaAs}, \mathrm{Al}_{\mathrm{x}} \mathrm{Ga}_{1-\mathrm{x}} \mathrm{As}$ and $\mathrm{In}_{\mathrm{y}} \mathrm{Ga}_{1-\mathrm{y}}$ As were prepared by molecular beam epitaxy and characterized by time-of-flight secondary-ion mass spectrometry and X-ray photoelectron spectroscopy. The direct patterning of features of width $\leq 50 \mathrm{~nm}$ on GaAs and $\mathrm{In}_{0.2} \mathrm{Ga}_{0.8}$ As surfaces is shown to be the result of the formation of a strongly bonded surface oxide induced under high electric-field conditions existing between the scan tip and the substrate. The significance of STM pattern generation of nanometerscale oxide masks for use in the fabrication of lowdimensional heterostructures is discussed.

[Contact: Wen F. Tseng, (301) 975-5291]

Parameswaran, M., Chung, R., Gaitan, M., Johnson, R.B., and Syrzycki, M., Commercial CMOS Fabricated Integrated Dynamic Thermal Scene Simulator, Technical Digest, 1991 International Electron Devices Meeting (IEDM), Washington, D.C., December 8-11, 1991, pp. 29.4.1-19.4.4 (1991).

We report a prototype integrated dynamic thermal scene simulator chip, consisting of a 2 by 2 array of integrated thermal pixels. The chips were fabricated using commercial CMOS processes available through vendor services. The micromachining process, needed to create the thermally isolated structures, is introduced as a maskless post-processing step. The thermal pixel and the control electronics are designed as a module for an easy implementation of the array. Test results indicate that the pixels have a thermal time constant of $5 \mathrm{~ms}$ and are capable of producing an infrared output of apparent radiometric temperatures in excess of $600{ }^{\circ} \mathrm{C}$ and color temperatures of at least $500{ }^{\circ} \mathrm{C}$. The control electronics is capable of switching within $900 \mathrm{~ns}$, enabling the addressing of multiple pixels within the $200-\mathrm{Hz}$ frame time required for a typical dynamic thermal scene simulation.

[Contact: Michael Gaitan, (301) 975-2070]

Pellegrino, J.G., Qadri, S., Tseng, W.F., and Comas J., Periodicities Associated with Low-Order AlAs/GaAs Superlattices, Journal of Thin Solid Films, Vol. 206, 


$$
\text { pp. } 40-46 \text { (1991). }
$$

The use of MBE to produce heterostructures has made it possible to examine superlattices with monolayer and submonolayer period spacings. In this work we examine the physical properties for the superlattice system (GaAs)n1(AlAs)n2/GaAs(001) for low values of $n 1$ and $n 2$, i.e., $n 1=n 2=3,6,12$. Normal, interrupted growth, and migration-enhanced epitaxy growth techniques were used to grow superlattice structures, and X-ray diffraction was used to analyze the major and satellite peak positions. An analysis of the major diffraction peaks and their associated satellites exhibited superlattice periodicity in good agreement with theory. Diffraction peaks were also observed in regions adjacent to the primary diffraction peaks which did not occur in the expected satellite positions. An analysis of these peaks relative to the primary peaks indicate periodicities which are greater than the intended period. One possible cause for these periodicities is variations in growth conditions which occur while the superlattice is being grown. An understanding of low-order superlattices is important for structures which are dependent upon interface sharpness.

[Contact: Joseph G. Pellegrino, (301) 975-2123]

\section{Plasma Processing}

\section{Released for Publication}

Sobolewski, M.A., Roberts, J.R., Olthoff, J.K., Whetstone, J.R., and Van Brunt, R.J., The GEC rf Reference Cell: A Parallel-Plate rf-System to Study Plasma-Processing Discharges.

A "Reference Cell" for generating radio frequency (rf) glow discharges in gases at a frequency of $13.56 \mathrm{MHz}$ is described. The reference cell provides an experimental platform for the intercomparison of plasma measurements which are carried out in a common reactor geometry, thereby enhancing the transfer of knowledge and insight gained in rf discharge studies. The results of performing ostensibly identical measurements on five of these cells in four different laboratories are analyzed and discussed. Measurements were made of plasma voltage and current characteristics for discharges in pure argon at specified values at applied voltages, gas pressures, and gas flow rates.

[Contact: James K. Olthoff, (301) 975-2431] $\underline{\text { Power Devices }}$

Released for Publication

Hefner, A.R., Jr., Semiconductor Measurement Technology: INSTANT: IGBT Network Simulation and Transient ANalysis Tool, to be published as NIST Special Publication 400-88.

The IGBT (Insulated Gate Bipolar Transistor) is a power semiconductor device that has gained acceptance among power electronic circuit design engineers for motor driver and power converter applications, due to its efficient voltage gate drive requirements and due to its high current density capability. These devices have the best features of both power MOSFETs and power bipolar transistors. When designing the circuits and systems that utilize IGBTs, circuit simulations are needed to examine the behavior of the IGBTs within the circuit. However, the semiconductor device models available in most circuit simulators were originally intended to describe microelectronic devices and cannot adequately describe the characteristics of power devices.

In this publication, a compact IGBT model suitable for incorporation in circuit simulators is described, and a circuit simulation program called INSTANT is presented that simulates the dynamic behavior of IGBTs within any external drive, load, and feedback circuit configuration. The INSTANT simulator solves the systems of differential equations (state equations) that describe each component of the circuit, where the equations for the individual components are coupled by the circuit configuration. The INSTANT software package is designed to provide the flexibility to change the external circuit configuration and model equations. The device and circuit parameters are also readily accessible, and the graphics output provides a real-time display of the waveforms as they are calculated.

This publication also describes the automated measurement methods developed to extract the IGBT device model parameters from terminal electrical measurements. It is shown that unlike parameter extraction for microelectronic devices, the dynamic characteristics must be used to characterize the IGBTs and to extract the model parameters. This occurs because the devices exhibit non-quasi-static behavior and because the dynamic waveforms contain 
many features that isolate different physical mechanisms, whereas the physical mechanisms are convoluted in the relatively simple steady-state characteristics. The unique features of the IGBT electrical characteristics are explained using the model, and the procedures used to verify the IGBT model are given.

[Contact: Allen R. Hefner, Jr., (301) 975-2071]

\section{Recently Published}

Blackburn, D.L., Failure Mechanisms and Nondestructive Testing of Power Bipolar and MOS Gated Transistors, Proceedings of the EPE - MADEP '91/MADEP Symposium on Materials and Devices for Power Electronics, 4th European Conference on Power Electronics and Applications, Florence, Italy, September 2-6, 1991, pp. 0-252 to 0-257.

Failure mechanisms and nondestructive testing of power bipolar and MOS-gated devices are discussed. Bipolar transistor failures are initiated at relatively low temperatures, and these devices can be tested nondestructively. Modern MOS-gated device failure is initiated at temperatures far in excess of those normally considered safe and cannot be tested nondestructively today. The key to nondestructive testing is the ability to sense the onset of failure and to then remove all power from the transistor before the device temperature rises high enough to cause damage.

[Contact: David L. Blackburn, (301) 975-2068]

\section{Photodetectors}

\section{Released for Publication}

Gallawa, R.L., Gardner, J.L., Nettleton, D.H., and Stock, K.D., Results of an International Intercomparison of Detector Responsivity at 1300 and 1550 $\mathbf{n m}$, to be published in the CPEM Digest, Conference on Precision Electromagnetic Measurements, Paris, France, June 9-12, 1992.

An international intercomparison of spectral responsivity measurements at wavelengths of interest to the optical communications community was recently completed. Thirteen countries participated in the test that was conducted in the course of a year. Results are presented. Agreement is at about the $1 \%$ level.
[Contact: Robert L. Gallawa, (303) 497-3761]

Geist, J., Chandler-Horowitz, D., Robinson, A.M., James, C.R., Kohler, R., and Goebel, R., Numerical Modeling of Silicon Photodiodes for High-Accuracy Applications, to be published as NISTIR 4592.

The purpose of this National Institute of Standards and Technology Interagency/Internal Report (NISTIR) is to provide the source code for two Turbo Pascal 5.5 programs and an MSDOS batch program, along with a paper that describes the programs and provides examples of their use. These three programs serve as a batch mode interface to support highaccuracy photodiode modeling with Version 2 of the semiconductor device modeling program optimized for solar cell modeling, and it is somewhat difficult to access the highest accuracy available from PC-1D through this interface. Part I describes PC-1D from the point of view of high-accuracy photodiode modeling and describes the programs that support its use in this application. Parts II and III present examples of the use of the programs described in Part I to model two different types of experiments performed on silicon photodiodes in various high-accuracy applications.

[Contact: Jon Geist, (301) 975-2066]

Geist, J., Luther, J.E., Novotny, D.B., and Vahakangas, J., Simple, Low-Contrast Thermal Resolution Test Target.

An easily constructed, thermal resolution test target for low-contrast applications is described. The calibration of the target need not be obtained by reference to some other radiometric standard, but can be obtained directly from the mechanical dimensions of the device and the thermal conductivity of fused silica. [Contact: Jon Geist, (301) 975-2066]

\section{Livigni, D., and Li, X., Spatial Uniformity Measure- ment of Optical Detector Response, to be published in the Proceedings of the Measurement Science Conference, Anahiem, California, January 30-31, 1992.}

The response of optical detectors to light can vary when the light illuminates different areas of the detector. A perfectly uniform detector would have no 
change in response when the illuminated area is varied, but the response of real detectors can vary significantly. A scanning system for measuring the response uniformity and methods of quantifying the degree of uniformity are described here. Surface plots and topographical maps of the measured response are presented along with a statistical treatment. Sampling theorem restrictions affecting the accuracy of the results are described. Scans of actual detectors are included to show the wide range of uniformity possible.

[Contact: David Livigni, (303) 497-5898]

\section{Recently Published}

Geist, J., Chandler-Horowitz, D., Kohler, R., Robinson, A.M., and James, C.R., Numerical Modeling of Short-Wavelength Internal Quantum Efficiency, Metrologia, Vol. 28, pp. 193-196 (1991).

Results of numerical modeling of short-wavelength, internal quantum efficiency of various types of silicon photodiode are presented. The important conclusions are: 1) Interpreting the data obtained from oxide-bias experiments with the help of numerical quantum efficiency models will provide higher accuracy than can be obtained from the conventional self-calibration formula. 2) For high-quality silicon photodiodes, the spectral shape of the internal quantum deficiency (one minus the quantum efficiency) is virtually independent of the density of charge trapped in the oxide and of the surface-recombination velocity at the oxide-silicon interface.

[Contact: Jon Geist, (301) 975-2066]

\section{$\underline{\text { Radiation Effects }}$}

Recently Published

Campisi, G.J., Roitman, P., and Shontz, G.J., The Role of Annealing Conditions on the Radiation Response of Backgate MOSFETs, Extended Abstract, Proceedings of the Third Workshop on Radiation-Induced and/or Process-Related Electrically Active Defects in Semiconductor-Insulator Systems, Research Triangle Park, North Carolina, September 10-13, 1991, pp. 171-172 (1991).

SIMOX (Separation by IMplantation of OXygen) is the principal SOI technology used for operation in a radiation environment. The suitability of SIMOX in the submicrometer regime on fully depleted devices depends on the radiation response of the dielectric-the beam synthesized buried oxide. In this study, SOI p-MOSFETs were fabricated on the SIMOX substrates annealed at various times and temperatures. The radiation-induced threshold shifts, $\Delta V_{b g}$, of the gate was determined as a function of substrate processing. Preliminary capacitor studies showed a reduction in the radiation-induced flat-band voltage by $40 \%$ when the anneal temperature was increased from 1275 to $1350{ }^{\circ} \mathrm{C}$. The role of anneal temperature on the device properties and radiation response of the buried oxide was investigated in this study. We have correlated the microstructure at the SIMOX interface to the radiation response of the MOSFETs.

[Contact: Peter Roitman, (301) 975-2077]

Other Semiconductor Metrology Topics

\section{Recently Published}

Parameswaran, M., Chung, R., Gaitan, M., Johnson, R.B., and Syrzycki, M., Commercial CMOS Fabricated Integrated Dynamic Thermal Scene Simulator, Technical Digest, 1991 International Electron Devices Meeting (IEDM), Washington, D.C., December 8-11, 1991, pp. 29.4.1-19.4.4 (1991).

We report a prototype integrated dynamic thermal scene simulator chip, consisting of a 2 by 2 array of integrated thermal pixels. The chips were fabricated using commercial CMOS processes available through vendor services. The micromachining process, needed to create the thermally isolated structures, is introduced as a maskless post-processing step. The thermal pixel and the control electronics are designed as a module for an easy implementation of the array. Test results indicate that the pixels have a thermal time constant of $5 \mathrm{~ms}$ and are capable of producing an infrared output of apparent radiometric temperatures in excess of $600{ }^{\circ} \mathrm{C}$ and color temperatures of at least $500{ }^{\circ} \mathrm{C}$. The control electronics is capable of switching within $900 \mathrm{~ns}$, enabling the addressing of multiple pixels within the $200-\mathrm{Hz}$ frame time required for a typical dynamic thermal scene simulation.

[Contact: Michael Gaitan, (301) 975-2070] 
SIGNAL ACQUISITION, PROCESSING, AND TRANSMISSION

DC and Low-Frequency Metrology

Released for Publication

Avramov, S., Oldham, N.M., Jarrett, D.G., and Waltrip, B.C., Automatic Inductive Voltage Divider Bridge for Operation From $10 \mathrm{~Hz}$ to $100 \mathrm{kHz}$, to be published in the Conference Record, Conference on Precision Electromagnetic Measurements (CPEM '92), Paris, France, June 9-12, 1992.

An automatic bridge to calibrate inductive voltage dividers from $10 \mathrm{~Hz}$ to $100 \mathrm{kHz}$ is described. The bridge is based on a programmable 30-bit binary inductive voltage divider with terminal linearity of 0.1 parts per million at $400 \mathrm{~Hz}$ (linearity degrades 10 parts per million at frequency extremes). Measurements of programmable test dividers can be completely automated via General Purpose Interface Bus (GPIB, also known as IEEE 448 bus standard) using software developed to align the bridge components and perform an auto balance.

[Contact: Nile M. Oldham, (301) 975-2408]

Dziuba, R.F., and Elmquist, R.E., Improvements in Resistance Scaling at NIST Using Cryogenic Current Comparators, to be published in the Conference Record, Conference on Precision Electromagnetic Measurements (CPEM '92), Paris, France, June 9-12, 1992.

Cryogenic current comparators (CCCs) are being used at NIST to verify Hamon-type resistance-scaling techniques from the $1-\Omega$ level to the $100-\Omega, 1-\mathrm{k} \Omega$, $6453.20-\Omega$, and $10-\mathrm{k} \Omega$ resistance levels. Measurements comparing the $100 / 1$ ratio of a CCC to that of a Hamon transfer standard agree to within 0.01 parts per million - the practical limit of accuracy for a Hamon standard. The higher ratio accuracies and higher sensitivities of CCC bridges will make it possible to lower the uncertainties associated with resistance scaling at NIST by a factor of two or more. [Contact: Ronald F. Dziuba, (301) 975-4239]

Huang, D.X., and Kinard, J.R., AC-DC Differences of High Voltage Thermal Converters, to be published in the Proceedings of the 1992 Measurement Science Conference, Anaheim, California, January 30-31,
1992.

For some high-voltage thermal converters (HVTCs), their ac-dc differences vary with age, test-timing sequence, and voltage level, especially for $1000-\mathrm{V}$ ranges at frequencies above the audio range. A major contributor to these variations is dielectric loss which varies with temperature, voltage, and frequency. The results of intercomparisons of HVTCs and certain methods to reduce ac-dc differences and their voltage coefficients are presented.

[Contact: Joseph R. Kinard, (301) 975-4250]

Jarrett, D.G., and Dziuba, R.F., Automated AC Bridge for the Measurement of Resistors Over the Frequency Range $10 \mathrm{~Hz}$ to $10 \mathrm{kHz}$, to be published in the Conference Record, Conference on Precision Electromagnetic Measurements (CPEM '92), Paris, France, June 9-12, 1992.

An automated, guarded ac Kelvin bridge has been developed for measuring the frequency dependence of precision resistors from the $1-\Omega$ to the $1-\mathrm{M} \Omega$ level over the frequency range of $10 \mathrm{~Hz}$ to $10 \mathrm{kHz}$. The main ratio arms consist of two-stage 30-bit binary inductive voltage dividers. A guard inductive voltage divider drives a RC network to provide a known phase compensation to balance the quadrature component of the bridge. A bridge substitution technique is used in which the unknown is compared to a standard of known impedance. The bridge resolution is better than 0.1 parts per million for the in-phase and quadrature components.

[Contact: Dean G. Jarrett, (301) 975-4240]

Kinard, J.R., Huang, D.X., and Novotny, D.B., Integrated Thin-Film Micropotentiometers, to be published in the Conference Record, Conference on Precision Electromagnetic Measurements (CPEM '92), Paris, France, June 9-12, 1992.

Using thin- and thick-film technology and micromachining of silicon, integrated micropotentiometers have been designed and fabricated for voltage ranges from 1 to $200 \mathrm{mV}$ up to $1 \mathrm{MHz}$ or higher.

[Contact: Joseph R. Kinard, (301) 975-4250]

Kinard, J.R., Huang, D.X., and Novotny, D.B., Multilayer Thin-Film Thermal Converters, to be published in the Conference Record, Conference on Precision Electromagnetic Measurements (CPEM 
'92), Paris, France, June 9-12, 1992.

Multilayer, thin-film multijunction thermal converters (MJTCs) are being produced at NIST. This paper describes the thermal and physical designs and materials chosen to reduce ac-dc differences. Experimental results on prototype converters are also given.

[Contact: Joseph R. Kinard, (301) 975-4250]

Kinard, J.R., Knight, R.B.D., Klonz, M., and de Vreede, J.P.M., Intercomparison of NIST, NPL, PTB, and VSL Thermal Voltage Converters from $100 \mathrm{kHz}$ to $1 \mathrm{MHz}$, to be published in the Conference Record, Conference on Precision Electromagnetic Measurements (CPEM '92), Paris, France, June 9-12, 1992.

Coaxial thermal voltage converters (TVCs) have been hand-carried between NIST, NPL, PTB, and VSL for intercomparison of ac-dc difference from $100 \mathrm{kHz}$ to $1 \mathrm{MHz}$. This paper briefly describes the methods and underlying principles on which ac-dc difference determinations are based in each laboratory and gives the results of the intercomparison.

[Contact: Joseph R. Kinard, (301) 975-4250]

Oldham, N.M., Waltrip, B.C., and Petersons, O., Automatic Impedance Bridge for Calibrating Standard Inductors, to be published in the Conference Record, Conference on Precision Electromagnetic Measurements (CPEM '92), Paris, France, June 912, 1992.

An impedance bridge that compares standard inductors to characterized resistors is described. A dual channel digitally synthesized source that is adjustable in amplitude and phase is used to balance the bridge. Uncertainties of less than \pm 100 parts per million are possible in low audio frequency range for inductors from $10 \mu \mathrm{H}$ to $10 \mathrm{H}$.

[Contact: Nile M. Oldham, (301) 975-2408]

Zhen, Z., Rebuldela, G., Kinard, J.R., Janik, D., Huang, D.X., and de Vreede, J., Intercomparison of Thermal Converters at NIM, NIST, PTB, SIRI, and VSL from 1 to $100 \mathrm{MHz}$, to be published in the Conference Record, Conference on Precision Electromagnetic Measurements (CPEM '92), Paris, France, June 9-12, 1992.

Coaxial, thermal voltage converters (TVCs) have been intercompared between NIM, NIST, PTB, SIRI, and VSL in the frequency range from 1 to $100 \mathrm{MHz}$. This paper briefly describes the methods and underlying principles on which rf-dc difference determinations are based in each laboratory and gives the results of the intercomparisons.

[Contact: Gregorio Rebuldela, (301) 975-3561]

\section{Recently Published}

Elmquist, R.E., and Dziuba, R.F., Isolated Ramping Current Sources for a Cryogenic Current Comparator Bridge, Review of Scientific Instrumentation, Vol. 62, No. 10, pp. 2457-2460 (October 1991).

The design and performance of a pair of highly isolated ramping and reversing direct current sources for use with a cryogenic current comparator resistance bridge and dc SQUID detector are described. The current sources are floating and isolated from each other, and are internally programmed to reverse the output current while maintaining the SQUID feedback control system in lock. Sources have been constructed with full-scale current ranges from 0.65 $\mathrm{mA}$ to $100 \mathrm{~mA}$ and have been used in the comparisons of precision standard resistors at the 0.01 partsper-million level.

[Contact: Randolph E. Elmquist, (301) 975-6591]

Waveform Metrology

\section{Released for Publication}

Oldham, N.M., and Hetrick, P.S., Characterized Generator Extends Phase Meter Calibrations From $50 \mathrm{kHz}$ to $20 \mathrm{MHz}$, to be published in the Conference Record, Conference on Precision Electromagnetic Measurements (CPEM '92), Paris, France, June 9-12, 1992.

A phase angle standard generator made by phase locking two function generators is described. The generator produces two sine waves that are programmable in phase $\left(0\right.$ to $\left.360^{\circ}\right)$, amplitude $(0$ to $40 \mathrm{~V}$ rms), and frequency ( $<1 \mathrm{~Hz}$ to $20 \mathrm{MHz}$ ). The phase linearity is characterized from \pm 50 to $\pm 250 \mathrm{mdeg}$ over the frequency range without external phase standards.

[Contact: Nile M. Oldham, (301) 975-2408]

Waltrip, B.C., Parker, M.E., Oldham, N.M., and Bell, 
B.A., A Sampling Technique for Calibrating Phase Angle Generators from $1 \mathrm{~Hz}$ to $100 \mathrm{kHz}$, to be published in the Conference Record, Conference on Precision Electromagnetic Measurements (CPEM '92), Paris, France, June 9-12, 1992.

A method of calibrating phase angle generators from $1 \mathrm{~Hz}$ to $100 \mathrm{kHz}$ is described. A commercial dualchannel waveform sampler is used to digitize both waveforms of the generator. The phase relationship between the two signals is resolved to $<1$ mdeg using a four-parameter sine fit. The uncertainty in phase linearity is 1 to 10 mdeg over the frequency range.

[Contact: Bryan C. Waltrip, (301) 975-2438]

\section{Recently Published}

Chesnut, S.M., and Paulter, N.G., Automatic Waveform Analysis and Measurement System User Manual, NISTIR 3978 (December 1991).

The theory and operation of an upgraded version of the National Institute of Standards and Technology (NIST) Automatic Waveform Analysis and Measurement System (AWAMS) is described. This system was commissioned by the Army Primary Standards Laboratory to facilitate measurement comparability with NIST. The AWAMS has been installed at Redstone Arsenal, Alabama.

[Contact: S. Michelle Chesnut, (303) 497-3456]

Oldham, N.M., Hetrick, P.S., Kramar, J., Penzes, W., Wheatley, T., and Teague, C., Electronic Limitations in Phase Meters for Heterodyne Interferometry, Proceedings of the Sixth Annual Conference of the American Society for Precision Engineering, Santa Fe, New Mexico, October 13-18, 1991, pp. 4749.

Reasonable attention has been given to the fidelity of the process by which heterodyne interferometers convert optical path difference between beams that have traversed a test leg and a reference leg, respectively, to a phase difference between electrical signals from the reference and test photodetectors. This paper reports on a study of the next step: to obtain a quantitative result from these signals by measuring the electrical phase difference between the two photodetector signals.

[Contact: Nile M. Oldham, (301) 975-2408]
Cryoelectronic Metrology

Released for Publication

Walsh, T., Zero-Bias Anomalies in the CurrentVoltage and Conductance-Voltage Characteristics of High-Critical-Temperature Superconductor Tunnel Junctions.

The occurrence of zero-bias anomalies (ZBAs) in the tunneling spectra of high-critical-temperature superconductors (HTS) is reviewed. Following an historical introduction, we summarize studies noting HTS ZBAs. The plausibility and implications of proposed explanations, including quasi-particle tunneling and phase diffusion, are discussed. Experiments are proposed to answer questions that remain regarding the source and significance of the ZBAs.

[Contact: Michael W. Cromar, (303) 497-5375]

\section{Recently Published}

Benz, S.P., and Burroughs, C.J., Two-Dimensional Arrays of Josephson Junctions as Voltage-Tunable Oscillators, Superconductor Science Technolgy, Vol. 4, pp. 561-567 (1991).

We have detected coherent emission from two-dimensional arrays of superconductor-insulator-superconductor Josephson junctions. Two-dimensional arrays emit coherent radiation over a frequency range of 60 to $210 \mathrm{GHz}$, when coupled to detector junctions through dc-blocking capacitors. The detector junctions exhibit Shapiro steps at frequencies corresponding to the voltage across single-array junctions. The maximum power from a 10 -by-10 junction array coupled to a detector junction occurs at $150 \mathrm{GHz}$ and is estimated to be $0.4 \mu \mathrm{W}$, based on simulations of the detector circuit. By varying the number of array junctions, the array geometry, the junction critical urrent, and the coupling circuit, we have begun determining the essential conditions for observing coherent emission.

[Contact: Sam P. Benz, (303) 497-5258]

Hamilton, C.A., and Gilbert, K.C., Margins and Yield in Single Flux Quantum Logic, IEEE Transactions on Applied Superconductivity, Vol. 1, No. 4, pp. 157-163 (December 1991).

Simulations are used to optimize the design of simple 
rapid-single-flux quantum (RSFQ) logic gates and to determine their margins. Optimizations based on maximizing the smallest (critical) margin result in critical margin values in the range 19 to $50 \%$. A Monte Carlo approach is used to illustrate the relationship between margins and process yield. Based on single gate results, the results show that one sigma parameter spreads of less than about $\pm 5 \%$ will be required to make medium- or large-scale integrated RSFQ logic circuits. Finally, a single-bit full adder using five RSFQ gates and a local self-timing network is simulated at the discrete component level. The full adder used $2000 \mathrm{~A} / \mathrm{cm}^{2}$ junctions with a specific capacitance of $0.04 \mathrm{pF} / \mu \mathrm{m}^{2}$ and had a logic delay of 87 ps and a worst-case margin of $\pm 10 \%$. These results show that margin calculations on individual gates are not necessarily representative of the performance of these gates in complex networks.

[Contact: Clark A. Hamilton, (303) 497-3740]

\section{Antenna Metrology}

[Also see Electromagnetic Interference - Radiated]

\author{
Released for Publication
}

Muth, L.A., Probe Position-Error Correction in Planar Near Field Measurements at $60 \mathrm{GHz}$ Experimental Verification.

This study was conducted to verify that the probeposition error-correction technique can be successfully applied to real data obtained on a planar near-field range, where the probe position errors are known. Since probe-position error correction is most important at high frequencies, measurements were made at $60 \mathrm{GHz}$. Six planar scans at $\mathrm{z}$ positions separated by $0.03 \lambda$ were obtained. The correction technique was applied to an error-contaminated near-field measurement constructed out of the six scans according to discretized periodic error functions. The results indicate that probe-position errors can be removed from real near-field data as successfully as from simulated data; some residual errors, which are thought to be due to multiple reflections, residual drift in the measurement system, and residual probe position errors in all three coordinates are observed. [Contact: Lorant A. Muth, (303) 497-3603]
Recently Published

Francis, M.H., and Wittmann, R.C., Swept Frequency Gain Measurements From 33 to $50 \mathrm{GHz}$ at the National Institute of Standards and Technology, Proceedings of the Antenna Measurement Techniques Association Meeting, Boulder, Colorado, October 7-11, 1991, pp. 1-3 to 1-16.

As part of an effort to provide improved measurement services at frequencies above $30 \mathrm{GHz}$, scientists at the National Institute of Standards and Technology have completed development of a swept frequency gain measurement service for the 33- to $50-\mathrm{GHz}$ band. This service gives gain values with an accuracy of \pm 0.3 $\mathrm{dB}$. In this paper, we discuss an example measurement and the associated errors.

[Contact: Michael H. Francis, (303) 497-5873]

Repjar, A.G., Kremer, D.P., Guerrieri, J.R., and Canales, N., Determining Faults on a Flat Phased Array Antenna Using Planar Near-Field Techniques, Proceedings of the Antenna Measurements Technique Association Meeting, Boulder, Colorado, October $7-11,1991$, pp. 8-11 to 8-19.

The Antenna Metrology Group of the National Institute of Standards and Technology (NIST) has recently developed and implemented measurement procedures to diagnose faults on a flat phased-array antenna. First, the antenna was measured on the NIST planar near-field (PNF) range, taking measurements on a plane where the multiple reflections between the probe and the antenna under test are minimized. This is important since the PNF method does not directly allow for their effects. Then, the NIST PNF software which incorporates the fast Fourier transform (FFT) was used to determine the antenna's gain and pattern, and to evaluate the antenna's performance. Next, the inverse FFT was used to calculate the fields at the aperture plane. By using this technique, errors in the aperture fields due to multiple reflections can be avoided. By analyzing these aperture plane data through the use of detailed amplitude and phase contour plots, faults in the antenna were located and corrected. The PNF theory and utilization of the inverse FFT is briefly discussed and results are shown.

[Contact: Andrew G. Repjar, (303) 497-5703] 
Noise Metrology

Released for Publication

Estin, A.J., Juroshek, J.R., Marks, R.B., Clague, F.R., and Allen, J.W., Basic rf and Microwave Measurements: A Review of Selected Programs at NIST.

This paper summarizes the principles, historical background, and present status of three primary areas of rf and microwave measurements and standards: circuit parameter measurement, power measurement, and noise generation and measurement. Both the reference standards and the techniques of measurement and of transfer to secondary standards are addressed. An extensive bibliography is provided to enable the interested reader to pursue areas to greater depth, and brief discussions to indicate likely directions of current and future work are included.

[Contact: John R. Juroshek, (303) 497-5362]

Perera, S., "Broadband Mismatch Error" in Noise Measurement Systems, to be published in the Conference Record, Conference on Precision Electromagnetic Measurements (CPEM '92), Paris, France, June 9-12, 1992.

Microwave noise measurement systems of a doublesided heterodyne design, with a wide bandwidth and an electrically long transmission line at the input, may suffer from a large error; systems with a high IF are especially vulnerable. Factors contributing to this error are identified, and measures to control it are described.

[Contact: Sunchana Perera, (303) 497-3546]

Wait, D.F., A Comparison of Three Techniques for the Precision Measurement of Amplifier Noise, to be published in the Conference Record, Conference on Precision Electromagnetic Measurements (CPEM '92), Paris, France, June 9-12, 1992.

This paper discusses three new measurement techniques and the experimental results for precision fourparameter amplifier noise measurements. Two different measurement systems were used with two different types of low-noise X-band amplifiers. The current measurement accuracy is about $\pm 0.2 \mathrm{~dB}$.

[Contact: David F. Wait, (303) 497-3610]
Microwave and Millimeter-Wave Metrology

Released for Publication

Estin, A.J., Juroshek, J.R., Marks, R.B., Clague, F.R., and Allen, J.W., Basic rf and Microwave Measurements: A Review of Selected Programs at NIST.

This paper summarizes the principles, historical background, and present status of three primary areas of $\mathrm{rf}$ and microwave measurements and standards: circuit parameter measurement, power measurement, and noise generation and measurement. Both the reference standards and the techniques of measurement and of transfer to secondary standards are addressed. An extensive bibliography is provided to enable the interested reader to pursue areas to greater depth, and brief discussions to indicate likely directions of current and future work are included. [Contact: John R. Juroshek, (303) 497-5362]

Furlow, R., Shimoda, R.Y., Williams, D.F., Marks, R.B., and Gupta, K.C., Benchmark for the Verification of Microwave CAD Software, to be published in the Proceedings of the 38th ARFTG Conference, San Diego, California, December 4-6, 1991.

A set of microstrip structures which constitute a comprehensive benchmark for the validation of microwave Computer-Aided-Design (CAD) software has been developed in a collaborative effort led by Boeing Defense and Space. The benchmark is designed so as to exhibit a wide range of physical mechanisms which may or may not be incorporated into commercial microwave CAD software. The structures are characterized experimentally with respect to a well-understood calibration in which the reference impedance has only a real component.

[Contact: Dylan F. Williams, (303) 497-3138]

\section{Marks, R.B., Comments on "Rapid Pulsed Microwave Propagation."}

This letter discusses a recently published paper which reports experimental evidence of electromagnetic pulses propagating faster than the speed of light. It argues that such results contradict Maxwell's equations. Limitations of the experiment are examined. [Contact: Roger B. Marks, (303) 497-3037]

Marks, R.B., and Williams, D.F., Traceability for On- 
Wafer MMIC Measurements, to be published in the Conference Record, Conference on Precision Electromagnetic Measurements (CPEM '92), Paris, France, June 9-12, 1992.

New considerations concerning the establishment of traceability for on-wafer measurements are explored. Peculiar features related to the small size of planar transmission lines, including significant attenuation and limited reproducibility, alter our view of traceability and how it is achieved. The role of the characteristic impedance is identified, and methods for its measurement are discussed.

[Contact: Roger B. Marks, (303) 497-3037]

Williams, D.F., and Marks, R.B., Reciprocity Relations in Waveguide Junctions.

The implications of expressions relating the forward and reverse transmission coefficients of a waveguide junction derived from the Lorentz reciprocity condition are explored. The two terms in the relation, the phase of the reference impedance in the guide and a new reciprocity factor, lead to an asymmetric scattering parameter matrix when one of the transmission lines connected to the junction is lossy.

[Contact: Dylan F. Williams, (303) 497-3138]

Williams, D.F., and Marks, R.B., Scattering Parameter Measurement Verification, to be published in the Conference Record, Conference on Precision Electromagnetic Measurements (CPEM '92), Paris, France, June 9-12, 1992.

A powerful new technique enables the verification of the measurement accuracy of scattering parameter calibrations. This technique determines the relative reference impedance, the reference plane offset, and the worst-case measurement deviations of any calibration in comparison to a standard calibration. Experimental results for on-wafer measurements are presented.

[Contact: Dylan F. Williams, (303) 497-3138]

Williams, D.F., Marks, R.B., and Davidson, A., Comparison of On-Wafer Calibrations, to be published in the Proceedings of the 38th ARFTG Conference, San Diego, California, December 4-6, 1991.

A powerful new technique enables the verification of the measurement accuracy of scattering parameter calibrations. This technique determines the relative reference impedance, reference plane offset, and the worst-case measurement deviations of any calibration in comparison to a benchmark calibration. The technique is applied to several popular on-wafer scattering parameter calibrations, and the deviations between those calibrations and the through-reflect line calibration are quantified.

[Contact: Dylan F. Williams, (303) 497-3138]

Williams, D.F., Marks, R.B., Walker, D.K., and Clague, F., Wafer Probe Transducer Efficiency.

The transducer efficiency of a microwave wafer probe is measured experimentally. The usual expressions employed to calculate the transducer efficiency in terms of measured scattering parameters are shown to be inaccurate. The correct expressions using results from a new circuit theory are derived and shown to agree with experiment.

[Contact: Dylan F. Williams, (303) 497-3138]

Zhen, Z., Rebuldela, G., Kinard, J.R., Janik, D., Huang, D.X., and de Vreede, J., Intercomparison of Thermal Converters at NIM, NIST, PTB, SIRI, and VSL from 1 to $100 \mathrm{MHz}$, to be published in the Conference Record, Conference on Precision Electromagnetic Measurements (CPEM '92), Paris, France, June 9-12, 1992.

Coaxial, thermal voltage converters (TVCs) have been intercompared between NIM, NIST, PTB, SIRI, and VSL in the frequency range from 1 to $100 \mathrm{MHz}$. This paper briefly describes the methods and underlying principles on which $\mathrm{rf}$-dc difference determinations are based in each laboratory and gives the results of the intercomparisons.

[Contact: Gregorio Rebuldela, (301) 975-3561]

Recently Published

Judish, R.M., and Burns, J.G., Measurement Program Compares Automatic Vector Analyzers [original title: ARFTG Sponsors Measurement Comparison Program], Microwaves \& RF, pp. 203-ff (May 1991).

The Automated Radio Frequency Techniques Group (ARFTG) has developed a program that provides ARFTG members the opportunity to compare the performance of their automatic network analyzers to 
that of their peers. This program is called the Measurement Comparison Program. Participants are provided an analysis of their measurement results in comparison to measurements made at other laboratories.

[Contact: Robert M. Judish, (303) 497-3380]

Wittmann, R.C., and Yaghjian, A.D., Spherical-Wave Expansions for Piston-Radiator Fields, Journal of the Acoustical Society of America, Vol. 90, No. 3, pp. 1647-1655 (September 1991).

Simple spherical-wave expansions for the continuouswave fields of a circular piston radiator in a rigid baffle are derived. These expansions are valid throughout the illuminated half space and are useful for efficient numerical computation in the near-field region. Multipole coefficients are given by closedform expressions which can be evaluated recursively. [Contact: Ronald C. Wittmann, (303) 497-3326]

\section{Electromagnetic Properties}

\section{Released for Publication}

Vanzura, E.J., Weil, C.M., and Williams, D.F., Complex Permittivity Measurements of GalliumArsenide Using a High-Precision Resonant Cavity, to be published in the Conference Record, Conference on Precision Electromagnetic Measurements (CPEM '92), Paris, France, June 9-12, 1992.

Data are presented on the complex permittivity of gallium arsenide, as measured at room temperature in the 8 - to $12-\mathrm{GHz}$ frequency range. The measurements were performed using a mode-filtered cylindrical cavity resonator with helically wound walls. The estimated accuracies are $\pm 0.4 \%$ in relative permittivity and $\pm 5 \%$ in loss tangent at $10 \mathrm{GHz}$.

[Contact: Eric J. Vanzura, (303) 497-5752]

\section{Recently Published}

Ondrejka, A.R., and Kanda, M., A Time-Domain Method for Measuring the Reflection Coefficient of Microwave Absorbers at Frequencies Below $1 \mathrm{GHz}$, 1991 Digest, Antennas and Propagation Society Symposium, London, Ontario, Canada, June 24-28, 1991, Vol. 3, pp. 1656-1663.

A wideband time-domain reflectometer is used to evaluate the reflection characteristics of $\mathrm{rf} / \mathrm{microwave}$ absorbers. The reflectometer uses an array of two identical broadband antennas, both transmitting and receiving. The method uses the two antennas in a difference mode to remove the undesired signals and enhance the small reflections being measured. Using this technique, we can separate front-surface reflections from those which are generated at greater angles. The bandwidth of our pulses is $30 \mathrm{MHz}$ to $1000 \mathrm{MHz}$, and reflection characteristics are measured over this range. The method has been used to characterize the reflectivity of three different types of absorber placed in an anechoic chamber. The results are reported together with the measurement accuracy. [Contact: Arthur R. Ondrejka, (303) 497-3309]

\section{$\underline{\text { Laser Metrology }}$}

\section{Recently Published}

Sanford, N.A., Malone, K.J., and Larson, D.R., Extended-Cavity Operation of Rare-Earth Doped Glass Waveguide Lasers [original title: ExtendedCavity Operation of Glass Integrated-Optic Lasers: Mode-Locking, Q-Switching, and Wavelength Tuning], Optics Letters, Vol. 16, No. 14, pp. 1095-1097 (July 15, 1991). [Proceedings of the Integrated Photonics Research Conference, Monterey, California, April 9-11, 1991.]

Integrated-optic lasers have been operated, pulsed and cw, using extended cavities. The results are: modelocked pulse widths of roughly $80 \mathrm{ps}, 1.2 \mathrm{~W}$ of Qswitched peak power, and tuning from 1050 to 1074 $\mathrm{nm}$ with approximately 7-GHz linewidth.

[Contact: Norman A. Sanford, (303) 497-5239]

\section{Optical Fiber Metrology}

\section{Released for Publication}

Franzen, D.L., Overview of Photonic Primary Standards Development, to be published in the Program Digest, DoD Fiber Optic Conference, McLean, Virginia, March 24-27, 1992.

The National Institute of Standards and Technology is working on primary standards to support lightwave communications. Primary standards are being developed to support the following measurements: absolute optical power, optical fiber geometry, and wavelength. 
Present capabilities and eventual goals of the various standards programs are discussed.

[Contact: Douglas L. Franzen, (303) 497-3346]

Obarski, G., Drapela, T., and Young, M., Scanning Confocal Microscopy for Finding Diameters of Circular Objects: Numerical Modelling, to be published in the Proceedings of SPIE (The International Society for Optical Engineering, P.O. Box 10, Bellingham, Washington 98227-0010), International Symposium on Laser Spectroscopy-Biomedical Optics-Time Resolved Laser Spectroscopy in Biochemistry 3, Los Angeles, California, January 20-22, 1992.

We calculated the image of a circular edge as determined by a scanning confocal microscope. In scalar theory, the quarter-intensity point of the edge response locates the geometrical-optics image of a straight edge. For a circular object, however, the quarter-intensity point is displaced from the geometrical-optics image of the edge according to the diameter of the object. For example, for an object that has a diameter of 21 resolution limits, the displacement error is $\approx 0.01$ resolution limits. We give the error that results from locating the quarter-intensity point for diameters as small as 1 resolution limit. The error will be even greater if the object is scanned off-axis. For example, the error for an object whose diameter is 21 resolution limits and which is scanned 3 resolution limits off-axis is $\approx 0.45$ resolution limits. Finally, we calculated displacement errors for vertical lines of width as small as 1 resolution limit.

[Contact: Gregory Obarski, (303) 497-5747]

Pal, B.P., Gallawa, R.L., and Goyal, I.C., LP ${ }_{11}$-Mode Leakage Loss Spectrum in Coated Depressed Index Clad Fibers.

A quantitative investigation of the leakage loss spectrum of the $\mathrm{LP}_{11}$-mode in coated depressed index clad fibers is made using the matrix method. The study confirms the oscillations seen in the experimental data of cut-off wavelength measurements. The results are compared with recently published results and a plausible explanation of the discrepancies is given. [Contact: Robert L. Gallawa, (303) 497-3761]

Young, M., Mechels, S.E., and Hale, P.D., Optical Fiber Geometry: Accurate Measurement of Cladding Diameter, to be published in the Conference
Record, Conference on Precision Electromagnetic Measurements (CPEM '92), Paris, France, June 9. 12, 1992.

This paper reports progress toward developing an artifact standard for video microscopes devoted to measuring optical fiber geometry. We have used a contact micrometer to measure fiber diameters with about $40 \mathrm{~nm}$, and we are developing a scanning confocal microscope and a white light interference microscope with which to verify the accuracy of the micrometer.

[Contact: Matt Young, (303) 497-3223]

\section{Recently Published}

Danielson B.L., Precise Length Measurements in Multimode Optical Fibers [original title: Precision Length Measurements in Multimode Optical Fibers], Applied Optics, Vol. 30, No. 27, pp. $3867-3872$ (20 September 1991).

By using selective optical excitation, both the group index and group delay of on-axis modes of multimode fibers can be determined with high precision. The group index of several types of fibers was measured at $1310 \mathrm{~nm}$ in a fiber Michelson interferometer, and the values tabulated. Group delays were obtained from the transit time of short duration optical pulses. From these data, the length of reference fibers about $2 \mathrm{~km}$ long was calculated. Length measurement accuracy was limited by group index uncertainties to about $0.04 \%$. Also, a technique is described which uses these reference fibers to minimize uncertainties in distance measurements made with multimode optical time domain reflectometers.

[Contact: Bruce L. Danielson, (303) 497-5620]

Gallawa, R.L., Goyal, I.C., and Ghatak, A.K., Optical Waveguide Analysis Using Modified Airy Functions, Fiber and Integrated Optics, Vol. 10, pp. 1-10 (1991).

We review a little-used but powerful method of solving one of the most fundamental equations of mathematical physics. The method is not new, but it is apparently not familiar to the optics community. It uses a modification of the well-known Airy functions, which are easily calculated on desktop computers. We review the method through examples which have an exact solution. We trust that this review will serve 
to stimulate further examination of a method that seems to have considerable promise.

The method that we review here is reminiscent of the WKBJ methodology (commonly known as the WKB approximation. WKBJ refers to the initials of four independent workers, Wentzel, Kramers, Brillouin, and Jeffrey, who first used the approximation procedure to solve the Schroedinger wave equation in one dimension), but the solution, although approximate, is much more useful than the traditional WKBJ solution and can be used with almost as much ease. The method is extremely powerful but, to our knowledge, is not being used by the optics community, where its use in analyzing optical fibers and integrated optical waveguides would be beneficial.

[Contact: Robert L. Gallawa, (303) 497-3761]

Mechels, S., and Young, M., Scanning Confocal Microscope for Precise Measurement of Optical Fiber Diameter, Proceedings of SPIE (The International Society for Optical Engineering, P.O. Box 10, Bellingham, Washington 98227-0010), Scanning Microscopy Instrumentation, Vol. 1556, pp. 164-170 (1992).

We have constructed and evaluated a scanning confocal microscope for the precise measurement of optical fiber cladding diameter. The system measures the fiber endface directly and differs from conventional microscopes in that it minimizes the systematic error due to partial coherence. The results obtained with the scanning confocal microscope are checked by comparison with those obtained from a contact micrometer and by measuring a chrome-on-glass Standard Reference Material provided by NIST, Gaithersburg. Fiber diameters can be measured with a random uncertainty of $40 \mathrm{~nm}$ and a systematic error estimated to be $40 \mathrm{~nm}$.

[Contact: Steve Mechels, (303) 497-5409]

Mechels, S., and Young, M., Video Microscope with Submicrometer Resolution, Applied Optics, Vol. 30, No. 16, pp. 2202-2211 (1 June 1991).

We have constructed and evaluated a video microscope with a $150 \times 150 \mu \mathrm{m}$ field of view for performing optical fiber geometry measurements. The microscope consists of a frame-transfer video camera, condensing and filtering optics, a 40X, $0.65 \mathrm{NA}$ microscope objective, and frame digitizing electronics.
Using simple digital algorithms, we measure distance with a random uncertainty of about $70 \mathrm{~nm}$ across the full field of view, but width measurements suffer from a systematic error between 0.1 and $0.2 \mu \mathrm{m}$.

[Contact: Steven Mechels, (303) 497-3223]

\section{Optical Fiber Sensors}

\section{Released for Publication}

Day, G.W., Deeter, M.N., and Rose, A.H., Faraday Effect Sensors: A Review of Recent Progress, to be published in the Proceedings of the International Conference on Optical Fiber Sensors, Wuham, China, October 9-11, 1991.

The last few years have seen dramatic progress in the development of Faraday effect sensors for measuring both magnetic fields and electric current. In the case of magnetic field sensors, the most significant advances have resulted from an investigation of new materials, especially the ferrimagnetic iron garnets. Minimum detectable magnetic fields have been reduced by several orders of magnitude to about 100 $\mathrm{pT} / \sqrt{\mathrm{Hz}}$ at $500 \mathrm{~Hz}$, and bandwidths of hundreds of megahertz have been obtained. In the case of electric current sensors, new methods of avoiding the effects of linear birefringence in optical fiber have resulted in much smaller and more stable sensors. One of those approaches, annealing of the fiber to reduce birefringence, has yielded sensors with temperature stabilities near that of the material limit, $<10^{-4} / \mathrm{K}$. These topics and other developments that have occurred since a previous summary [Proc. SPIE 985 138-150 (1988)] are discussed in this review.

[Contact: Gordon W. Day, (303) 497-5204]

Wolfe, R., Gyorgy, E.M., Lieberman, R.A., Fratello, V.J., Licht, S.J., Deeter, M.N., and Day, G.W., High Frequency Magnetic Field Sensors Based on the Faraday Effect in Garnet Thick Films.

The Faraday effect in the thick epitaxial films of magnetic garnets of the type used in magneto-optic isolators can be used as the basis for a fiber-optic magnetic field sensor. These films have uniaxial anisotropy perpendicular to the surface, and they contain bismuth to enhance the Faraday rotation. The typical magnetic domain pattern of meandering stripes changes in response to an applied field perpendicular to the film, and this changes the polar- 
ization of infrared light propagating perpendicular to the film. Theory and experiment show that the speed of operation is limited by relaxation or resonance effects to upper frequencies between $10^{6}$ and $10^{9} \mathrm{~Hz}$. Maximum sensitivity requires low magnetic moment and large thickness in conflict with the requirements for high speed.

[Contact: Merritt N. Deeter, (303) 497-5400]

Recently Published

Deeter, M.N., Rose, A.H., Day, G. W., and Samuelson, S., Sensitivity Limits to Ferrimagnetic Faraday Effect Magnetic Field Sensors, Journal of Applied Physics, Vol. 70, No. 10, pp. 6407-6409 (15 November 1991).

Magnetic field sensors based on the Faraday effect in bulk ferrimagnetic iron garnets exhibit many desirable characteristics, including high sensitivity and a large bandwidth. In general, the sensitivity of these sensors is a function of both the crystal geometry and composition. The geometrical dependence of the sensitivity in nonellipsoidal crystals, such as cylinders, is complicated by their spatially nonuniform demagnetizing factors. We compare sensitivity data obtained from a variety of cylindrical iron garnet samples with two models which predict the effective demagnetizing factor $\mathrm{N}_{\text {eff }}$ as a function of the length-to-diameter ratio. With respect to composition, we present experimental results of sensitivity versus diamagnetic substitution in the iron garnet series $\mathrm{Y}_{3} \mathrm{Fe}_{5-\mathrm{x}} \mathrm{Ga}_{\mathrm{x}} \mathrm{O}_{12}$. As expected, the sensitivity rises sharply as $\mathrm{x}$ approaches the compositional compensation point.

[Contact: Merritt N. Deeter, (303) 497-5400]

Rose, A.H., and Day, G.W., Optical Fiber Voltage Sensors for Broad Temperature Ranges, Proceedings of SPIE (The International Society for Optical Engineering, P.O. Box 10, Bellingham, Washington 98227-0010), Fiber Optic Components and Reliability, Vol. 1580, pp. 95-103 (1991).

In this paper, we describe the development of an optical fiber ac voltage sensor for aircraft and spacecraft applications. Among the most difficult specifications to meet for this application is a temperature stability of $\pm 1 \%$ from $-65^{\circ} \mathrm{C}$ to $+125^{\circ} \mathrm{C}$. This stability requires a careful selection of materials, components, and optical configuration with further compensation using an optical fiber temperature sensor located near the sensing element. The sensor is a polarimetric design, based on the linear electrooptic effect in bulk bismuth germanate $\left(\mathrm{Bi}_{4} \mathrm{Ge}_{3} \mathrm{O}_{12}\right)$. The temperature sensor is also polarimetric, based on the temperature dependence of the birefringence of bulk $\mathrm{SiO}_{2}$. The temperature sensor output is used to automatically adjust the calibration of the instruments. [Contact: Allen H. Rose, (303) 497-5599]

Williams, P.A., Clark, N.A., Blanca Ros, M., Vohra, R.T., Walba, D. M., and Wand, M. D., Large Electroclinic Effect in New Liquid Crystal Material, Proceedings of the Third International Conference on Ferroelectric Liquid Crystals, Boulder, Colorado, June 23-28, 1991, Vol. 121, pp. 143-146.

We report a new liquid crystal material (W317) which has an unusually large electroclinic effect in a phase tentatively identified as the smectic A. We show electroclinic tilt angles as large as $21^{\circ}$, and measurable tilt angles over a $40^{\circ} \mathrm{C}$ temperature range.

[Contact: Paul A. Williams, (303) 497-3805]

Williams, P.A., Rose, A.H., Day, G.W., Milner, T.E., and Deeter, M.N., Temperature Dependence of the Verdet Constant in Several Diamagnetic Glasses, Applied Optics, Vol. 30, No. 10, pp. 1176-1178 (1 April 1991).

We report measurements of the temperature dependence of the Verdet constant of $\mathrm{SiO}_{2}, \mathrm{SF}-57$, and BK7 glasses. In each case, the Verdet constant increases with temperature by the order of 1 part in $10^{4} / \mathrm{K}$ over the range from room temperature to $150{ }^{\circ} \mathrm{C}$. The results for each glass are within 3 to $20 \%$ of estimates obtained using the Becquerel formula with published data for dispersion and the change in index of refraction with temperature.

[Contact: Paul A. Williams, (303) 497-3287]

\section{Electro-Optic Metrology}

\section{Released for Publication}

Christensen, D.H., Pellegrino, J.G., Hickernell, R.K., and Tseng, W.F., Vertical-Cavity Surface-Emitting Laser with Distributed Quantum Wells.

We have observed lasing in single-crystal epitaxial GaAs-AlGaAs vertical-cavity distributed feedback structures with distributed quantum-well gain regions. 
The distributed feedback structure contains a quarterwave phase shift region at the center. The distributed gain is provided by quantum wells placed at the index discontinuity interfaces of the structure and a single quantum well at the center of the half-wavelengthlong phase slip region. We report initial findings of superradiant-like spectra which significantly narrows with increase in CW optical pump intensity, producing less than 0.3-nm full-width-half-maximum emission at $850.7 \mathrm{~nm}$.

[Contact: David H. Christensen, (303) 497-3354]

Franzen, D.L., Overview of Photonic Primary Standards Development, to be published in the Program Digest, DoD Fiber Optic Conference, McLean, Virginia, March 24-27, 1992.

The National Institute of Standards and Technology is working on primary standards to support lightwave communications. Primary standards are being developed to support the following measurements: absolute optical power, optical fiber geometry, and wavelength. Present capabilities and eventual goals of the various standards programs are discussed.

[Contact: Douglas L. Franzen, (303) 497-3346]

\section{Pal, B.P., Guided Wave Optics on Silicon: Physics, Technology, and Status.}

A semi-tutorial state-of-the-art review of silicon-based guided wave optics is presented. Starting from the physical principles behind waveguidance in planar and rectangular geometries, different technologies of silicon-based optical waveguides are described. A survey of various components/devices reported to date is also presented. A unified description of the physics and technology of silicon-based guided wave optics has been attempted. We believe it will be useful in the design, analysis, and realization of guided wave devices on silicon.

[Contact: Bishnu Pal, (303) 497-3931]

Takara, H., Kawanishi, S., Saruwatari, M., and Schlager,J.B.,Multi-Wavelength Birefringent-Cavity Mode-Locked Fiber Laser, to be published in the Technical Digest, Conference on Lasers and ElectroOptics, Anaheim, California, May 10-15, 1992.

A novel multi-wavelength mode-locked Er3+-doped fiber ring lase-r is first demonstrated using $45^{\circ}$ concatenated birefringent fibers in the cavity. Simultaneous four wavelength optical pulses with approximately 0.9 -nm separations and 30 -ps pulse durations were obtained.

[Contact: John B. Schlager, (303) 497-3542]

\section{Recently Published}

Boisrobert, C.Y., Franzen, D.L., Danielson, B.L., and Christensen, D.H., Low Coherence Optical Reflectometry of Laser Diode Waveguides, Proceedings of SPIE (The International Society for Optical Engineering, P.O. Box 10, Bellingham, Washington 98227-0010), Optical Technology for Signal Processing, Vol. 1474, pp. 285-290 (1991).

Laser diode waveguides are probed using low-coherence optical reflectometry. Reflections from the launch optics, front facet, and rear facet are located with a resolution of approximately $10 \mu \mathrm{m}$. Diodes mounted in pigtailed packages and on chip carriers have been studied.

[Contact: Christensen Y. Boisrobert, (303) 497-5052]

Gallawa, R.L., Goyal, I.C., and Ghatak, A.K., Optical Waveguide Analysis Using Modified Airy Functions, Fiber and Integrated Optics, Vol. 10, pp. 1-10 (1991).

We review a little-used but powerful method of solving the scalar wave equation. It uses a modification of the well-known Airy functions, which are easily calculated on desktop computers. The techniques are reminiscent of the WKBJ methodology (WKBJ refers to the initials of four independent workers, Wentzel, Kramers, Brillouin, and Jeffrey, who first used the approximation procedure to solve the Schroedinger wave equation in one dimension), but the solution, although approximate, is much more useful than the traditional WKBJ solution and can be used with almost as much ease. The method is extremely powerful but, to our knowledge, is not used in the optics community. It is useful in analyzing integrated optical waveguide components.

[Contact: Robert L. Gallawa, (303) 497-3761]

Ghatak, A.K., Gallawa, R.L., and Goyal, I.C., Modified Airy Function and WKB Solutions to the Wave Equation, NIST Monograph 176 (November 1991).

The purpose of this monograph is to revisit a basic equation of mathematical physics, $d^{2} \Psi / d x^{2}+$ 
$\Gamma^{2}(\mathrm{x}) \Psi(\mathrm{x})=0$, and to give approximate solutions based on the WKB method (WKB refers to the initials of three independent workers -- Wentzel, Kramers, Brillouin -- who first used the approximation procedure to solve the Schroedinger wave equation in one dimension) and on a modification of the Airy function. All of the examples used to illustrate the methods are based on optical waveguides and quantum mechanical problems. We hope that this monograph will prove to be tutorial, giving insight and understanding to the use of Airy functions in addressing the scalar-wave equation.

[Contact: Robert L. Gallawa, (303) 497-3761]

Goyal, I.C., Gallawa, R.L., and Ghatak, A.K., An Approximate Solution to the Scalar Wave Equation for Optical Waveguides, Applied Optics, Vol. 30, No. 21, pp. 2985-2989 (20 July 1991).

We consider an approximate solution to the onedimensional scalar wave equation appropriate to the planar optical waveguides often encountered in practice. The refractive index profile may be arbitrary. The method described here is more accurate and useful than the WKB method, which has often been applied to problems of this type, as unlike the WKB method, this method is valid even at turning points (WKB refers to the initials of three independent workers -- Wentzel, Kramers, Brillouin -- who first used the approximation procedure to solve the Schroedinger wave equation in one dimension). The fields and the propagation constants for the lowest order modes for two profiles are calculated and compared with the exact solution.

[Contact: Robert L. Gallawa, (303) 497-3761]

Grossman, E.N., Sauvageau, J.E., and McDonald, D.G., Lithographic Spiral Antennas at Short Wavelengths, Applied Physics Letters, Vol. 59, No. 25, pp. 3225-3227 (16 December 1991).

We have extended the high efficiency of lithographic antennas to mid-infrared wavelengths. Pattern measurements made at $9.5-\mu \mathrm{m}$ wavelength on a $65^{\circ}$, self-complementary, spiral antenna exhibit a ratio of response to orthogonal linear polarizations of $1.35 \mathrm{~dB}$, a beamwidth of $85^{\circ}$ ( $3 \mathrm{~dB}$ full width), a directivity of $8.2 \mathrm{~dB}$, and surprisingly, a close resemblance to the theoretical pattern for a $65^{\circ}$ spiral in free space. Direct detection measurements made with an ambient temperature blackbody source yield an antenna efficiency of $52 \pm 7 \%$, when corrected for incomplete filling of the antenna beam by the source, at a mean effective wavelength of $19 \mu \mathrm{m}$.

[Contact: Erich N. Grossman, (303) 497-5102]

Obarski, G.E., Lambdameter for Accurate Stability Measurements of Optical Transmitters, Proceedings of SPIE (The International Society for Optical Engineering, P.O. Box 10, Bellingham, Washington 98227-0010), Laser Testing and Reliability, Vol. 1620, pp. 41-48 (1991).

Very wavelength-stable, single-mode laser diodes will play an important role in near-future optical fiber communications systems. Two such applications are sources for dense wavelength multiplexing and local oscillators in coherent systems. To accurately measure wavelength of 1.3 - and $1.5-\mu \mathrm{m}$ single-mode sources, we developed a lambdameter that can also be used in the near IR and red regions of the spectrum. Wavelength accuracy and resolution are $\approx 0.1$ parts per million at the $0.63-\mu \mathrm{m}$ HeNe laser emission line. They were measured by comparing each of two adjacent modes of a HeNe laser, frequency-stabilized by a polarization technique, with a single mode from a second frequency-stabilized $\mathrm{HeNe}$ laser. We also verified the wavelength of the reference laser to 1 part-per-million accuracy by comparing it with the $1.52-\mu \mathrm{m} \mathrm{HeNe}$ laser emission line. The uncertainty in wavelength of the $1.52-\mu \mathrm{m} \mathrm{HeNe}$ laser is limited to the width of the Doppler gain curve whose peak is known within 0.2 parts per million. We describe our lambdameter and the performance of its reference laser as a wavelength transfer standard. Measurements on a commercially packaged $1.52-\mu \mathrm{m}$ DFBlaser diode transmitter show that its wavelength fluctuates by at least 1 part per million during normal changes in room temperature.

[Contact: Gregory E. Obarski, (303) 497-5747]

Sanford, N.A., Malone, K.J., Larson, D.R., and Hickernell, R.K., Y-Branch Waveguide Glass Laser and Amplifier, Optics Letters, Vol. 16, No. 15, pp. 1168-1170 (August 1, 1991).

A Y-branch channel waveguide laser operating near $1057 \mathrm{~nm}$ was fabricated in Nd-doped silicate glass by means of electric-field-assisted ion exchange. The overall length was $24 \mathrm{~mm}$. Optical pumping was performed with a continuous wave Ti:sapphire laser. Mirrors were bonded directly to the polished wave- 
guide facets. Using a 4\%-transmitting output coupler, the slope efficiency was $5.1 \%$; threshold was reached at 26-mW absorbed pump power. When operated as a single-pass $Y$-branch amplifier, the small-signal gain was $0.034 \mathrm{~dB} / \mathrm{mW}$. The $3-\mathrm{dB}$ splitting loss of the Y-branch structure was overcome when the absorbed pump power was approximately $85 \mathrm{~mW}$.

[Contact: Norman A. Sanford, (303) 497-5239]

Schlager, J.B., Kawanishi, S., and Saruwatari, M., Dual Wavelength Pulse Generation Using a ModeLocked Erbium-Doped Fiber Ring Laser, Electronics Letters, Vol. 27, No. 22, pp. 2072-2073 (24 October 1991).

Dual pulses, each with durations as short as 2 ps and different peak wavelengths, were concurrently produced with an actively mode-locked, erbium-doped fiber ring laser made in part with birefringent polarization-maintaining fiber. Peak-wavelength separation was proportional to the percentage of cavity having properly aligned birefringent fiber.

[Contact: John B. Schlager, (303) 497-3542]

Veasey, D.L., Hickernell, R.K., Larson, D.R., and Batchman, T.E., Waveguide Polarizers With Hydrogenated Amorphous Silicon Claddings, Applied Optics, Vol. 16, No. 10, pp. 717-719 (May 15, 1991).

We have fabricated TE- and TM-pass waveguide polarizers with polarization extinction ratios of 42 and $35 \mathrm{~dB}$, respectively. The devices were fabricated by the growth of hydrogenated amorphous silicon claddings on $\mathrm{K}^{+}-\mathrm{Na}^{+}$ion-exchanged channel waveguides in glass. Cladding thicknesses were accurately tuned to permit optimum coupling of either a TE or TM mode to the cladding. We have also demonstrated that waveguide losses of at least $760 \mathrm{~dB} / \mathrm{cm}$ can be measured using a photothermal deflection technique. [Contact: David L. Veasey, (303) 497-3439]

Veasey, D.L., Larson, D.R., and Batchman, T.E., InSitu Optimization of Coupling to Semiconductor Claddings from Dielectric Waveguides, Journal of Applied Physics, Vol. 68, No. 7, pp. 3753-3755 (1 October 1990).

We have experimentally verified the periodic dependence of coupling between lossless modes of dielectric waveguides and lossy modes supported by semiconductor waveguide claddings as a function of cladding thickness. Results were obtained by the in-situ monitoring of attenuation during the growth and etching of hydrogenated amorphous silicon on polarization-preserving, D-shaped, optical fiber. Strong correlation exists between theoretically predicted and experimental results for both TE and TM polarizations. The attenuation-monitoring technique allows for precise control of waveguide-cladding characteristics so that clad-waveguide devices, such as polarizers and detectors, can be optimized for better performance.

[Contact: David L. Veasey, (303) 497-3439]

\section{Complex System Testing}

\section{Recently Published}

Stenbakken, G.N., and Souders, T.M., Linear Error Modeling of Analog and Mixed-Signal Devices, Proceedings of the 1991 International Test Conference, Nashville, Tennessee, October 26-30, 1991, pp. 573-581.

Techniques are presented for developing linear error models for analog and mixed-signal devices. Methods for choosing parameters and assuring the models are complete and well-conditioned are included. Once established, the models can be used in a comprehensive approach for optimizing the testing of the subject devices.

[Contact: Gerard N. Stenbakken, (301) 975-2440]

\section{Other Signal Topics}

\section{Released for Publication}

Capobianco, T.E., Real-Time Eddy Current Monitoring Of Fatigue Crack Growth, to be published in the Proceedings of the Review of Progress in Quantitative Nondestructive Evaluation Conference, Brunswick, Maine, July 28-August 2, 1991.

We describe the use of a differential eddy current probe to monitor the growth of a fatigue crack from a starter notch in real time. The cracks for this study were grown in aluminum flat plate specimens using small drilled holes as starter notches. The specimens were fatigued in four-point bending. The probe consists of a circular outer drive coil containing a pair of inner pickup coils connected in series opposing and is glued on top of the starter notch. The data show 
that the probe is sensitive not only to the actual microscopic fatigue crack growth but also to microscopic events leading to the formation of the crack. [Contact: Thomas E. Capobianco, (303) 497-3141]

\section{Recently Published}

Danielson, B.L., and Boisrobert, C.Y., Absolute Optical Ranging Using Low Coherence Interferometry, Applied Optics, Vol. 30, No. 21, pp. 2975-2979 (20 July 1991).

We describe a method for measuring submicrometer distances with an asymmetric fiber Michelson interferometer having an LED as a source of radiation. By measuring the phase slope of the Fourier components in the frequency domain, it is possible to locate the position of reflections with nanometer precision even in the presence of sample dispersion. The method is compatible with time-domain sampling at the Nyquist rate which ensures efficiency in data acquisition and processing.

[Contact: Bruce L. Danielson, (303) 497-5620]

Grossman, E.N., Sauvageau, J.E., and McDonald, D.G., Lithographic Spiral Antennas at Short Wavelengths, Applied Physics Letters, Vol. 59, No. 25, pp. 3225-3227 (16 December 1991).

We have extended the high efficiency of lithographic antennas to mid-infrared wavelengths. Pattern measurements made at $9.5-\mu \mathrm{m}$ wavelength on a $65^{\circ}$, self-complementary, spiral antenna exhibit a ratio of response to orthogonal linear polarizations of $1.35 \mathrm{~dB}$, a beamwidth of $85^{\circ}$ ( $3 \mathrm{~dB}$ full width), a directivity of $8.2 \mathrm{~dB}$, and surprisingly, a close resemblance to the theoretical pattern for a $65^{\circ}$ spiral in free space. Direct detection measurements made with an ambient temperature blackbody source yield an antenna efficiency of $52 \pm 7 \%$, when corrected for incomplete filling of the antenna beam by the source, at a mean effective wavelength of $19 \mu \mathrm{m}$.

[Contact: Erich N. Grossman, (303) 497-5102]

Ma, M.T., and Adams, J.W., Phase Characteristics and Time Responses of Unknown Linear Systems Determined from Measured CW Amplitude Data [original title: Determination of Phase Characteristics and Time Responses of Unknown Linear Systems Based on Measured cw Amplitude Data], NIST Technical Note 1349 (November 1991). [A con- densed version, System Response to Pulsed Excita. tions Estimated From Measurement of cw Amplitudes, will be published in the Record of the 1992 International Symposium on Electromagnetic Compatibility, Beijing, China, May 25-27, 1992.]

An alternative technique is described for calculating the complete time and frequency characteristics of an unknown linear system from the measured amplitude response to continuous-wave (cw) excitations by assuming that the system transfer function is minimum phase. The time-response level so determined shows that the susceptibility of the system to damage by pulsed excitation is the greatest during the initial period of excitation. Other possible time responses, when the actual system transfer function is nonminimum phase, are also identified and analyzed [Contact: Mark T. Ma, (303) 497-3800]

\section{ELECTRICAL SYSTEMS}

Power Systems Metrology

\section{Released for Publication}

Fenimore, C., and Zhang, Y.X., Summary of 1989 Panel Session Paper on Robust Estimation in Parameter Extraction, to appear as a part of "Digital Techniques in HV Tests: Summary of 1989 Panel Session" in IEEE Transactions on Power Delivery.

This paper presents a method of evaluating highvoltage test parameters from digital records. It considers the problems of evaluating the parameters of the step response of an impulse measuring system. The method used should be robust; that is, the estimates should not be sensitive to noise or small variations in the input. Using cubic splines to fit the waveform provides an objective interpretation of "the mean curve" and "the steepest tangent line" and of the derived time parameters of the step response. Direct application of the standard definitions gives estimates which are sensitive to the small changes in the data. Redefining the tangent as the best-fitting straight line along the rising portion of the step gives robust estimates of the parameters.

[Contact: Charles Fenimore, (301) 975-2428]

Martzloff, F.D., Performance Criteria for Power System Compatibility, to be published in the Proceedings of the 1992 IEEE Applied Electronics 
Conference, Boston, Massachusetts, February 23-27, 1992.

Power electronics create an opportunity for better utilization of electric energy but can become a source of problems if the electromagnetic characteristics (immunity and emissions limits) of the equipment are not compatible with the characteristics (avoidable and unavoidable disturbances) of the power supply. Equipment performance criteria can help the end-user obtain better compatibility, reliability, and cost effectiveness of the equipment-power supply combination. [Contact: Francois D. Martzloff, (301) 975-2409]

Misakian, M., and IEEE Magnetic Fields Task Force (Members of the AC Fields Working Group in the IEEE Power Engineering Society), A Protocol for Spot Measurements of Residential Power Frequency Magnetic Fields.

This paper describes a simple protocol for measuring power frequency magnetic fields in residences. The protocol should not be interpreted as an IEEE standard, but if followed on a trial basis will provide for a degree of procedural uniformity which currently does not exist. The measurement procedures are simple and are not intended to characterize the temporal and spatial variability of magnetic field levels in residences. The protocol contains a structured component during which measurements are performed at specific locations and an unstructured component during which measurements are performed at locations requested by the occupants. The protocol requires personnel performing the measurements to explain the metrology-related limitations associated with the measurement results. This paper also discusses terminology related to power frequency magnetic fields and their measurement, the meaning of measurement accuracy and variability within the context of residential magnetic field measurements, and examples of measurement approaches that characterize the variability of magnetic field levels. [Contact: Martin Misakian, (301) 975-2426]

Van Brunt, R.J., Partial Discharge Pulse-Height Analysis - Promises and Limitations, to be published in the Proceedings of the IEEE International Symposium on Digital Techniques in High Voltage Measurements, Toronto, Canada, October 28-30, 1991.
An alternative approach to measurement of the phase-resolved stoachastic properties of partialdischarge pulses is described which can be used to unravel significant phase-to-phase memory propagation effects that give rise to nonstationary behavior in the observed pulse-height or phase-of-occurrence distributions. Examples are shown of data obtained using a point-to-dielectric discharge gap.

[Contact: Richard J. Van Brunt, (301) 975-2425]

Wan, H.-X., Moore, J.H., Olthoff, J.K., and Van Brunt, R.J., Electron Scattering and Dissociative Attachment by $\mathrm{SF}_{6}$ and Its Electrical-Discharge ByProducts.

Discrete electron-molecule processes relevant to $\mathrm{SF}_{6}$ etching plasmas are examined. Absolute, total scattering cross sections for $0.2-$ to $12-\mathrm{eV}$ electrons on $\mathrm{SF}_{6}$, $\mathrm{SO}_{2}, \mathrm{SOF}_{2}, \mathrm{SO}_{2} \mathrm{~F}_{2}, \mathrm{SOF}_{4}$, and $\mathrm{SF}_{4}$, as well as cross sections for negative-ion formation by attachment of 0.2- to 8-eV electrons have been measured. These are used to calculate dissociative-attachment rate coefficients as a function of $\mathrm{E} / \mathrm{N}$ for $\mathrm{SF}_{6}$ by-products in $\mathrm{SF}_{6}$. [Contact: James K. Olthoff, (301) 975-2431]

\section{Recently Published}

Anderson, W.E., Editor, Research for Electric Energy Systems - An Annual Report, NISTIR 4691 (December 1990).

This report documents the technical progress in the four investigations which make up the project "Support of Research Projects for Electrical Energy Systems," Department of Energy Task Order Number 137, funded by the U.S. Department of Energy and performed by the Electricity Division of the National Institute of Standards and Technology (NIST). The first investigation is concerned with the measurement of magnetic fields in support of epidemiological and in-vitro studies of biological field effects. NIST cohosted a workshop on exposure and biological parameters that should be considered during in-vitro studies with extremely low-frequency magnetic and electric fields. Additionally, equations were developed to predict the magnetic field in a parallel-plate magnetic-field exposure system. An IEEE standard prepared at NIST on measuring dc electric fields and ion-related parameters was approved and published. Various site visits were made to characterize the electric and magnetic fields in biological exposure 
systems. The second investigation is concerned with two different activities: the detection of trace levels of $\mathrm{S}_{2} \mathrm{~F}_{10}$ in $\mathrm{SF}_{6}$ and the development of an improved stochastic analyzer for pulsating phenomena (SAPP). The detection of $\mathrm{S}_{2} \mathrm{~F}_{10}$ in the presence of $\mathrm{SF}_{6}$ using mass-spectrometric detection coupled to a gas chromatograph is difficult because of the similar mass spectra. Enrichment techniques, capable of sub parts per billion detection in the case where the background gas is not $\mathrm{SF}_{6}$, are unsuitable for this application. $\mathrm{A}$ technique is described that enables the detection of $\mathrm{S}_{2} \mathrm{~F}_{10}$ in gaseous $\mathrm{SF}_{6}$ down to the parts per billion level using a modified gas chromatograph-mass spectrometer. The SAPP has been improved to allow direct real-time measurements of conditional pulseamplitude distributions of higher order than was previously possible. The new system was applied to an investigation of the stochastic behavior of negative corona (Trichel pulses) and the effect of a dielectric barrier on these discharges. The third investigation is concerned with breakdown and prebreakdown phenomena in liquid dielectrics. The activity reported here was a study of negative streamers preceding electric breakdown in hexanes. Using the imagepreserving optical delay, the growth of the streamers associated with partial discharges at a point cathode are photographed at high magnification. Simultaneous discharge current measurements enables a detailed description of the temporal and spatial development of streamers and provide a basis for the evaluation of models for the initiation of negative streamers. The last investigation is concerned with the evaluation and improvement of methods for measuring fast transients in electrical power systems such as might be associated with an electromagnetic impulse. The new draft of IEC-60 recommends the use of an independent reference measurement system to verify the performance of the impulse voltage measuring system under test. A compact resistive divider, NIST4, was designed for this purpose. It is anticipated that this divider, together with some Kerr electro-optical devices, will be used as the reference system at NIST. The design details of NIST 4 and its measurement capabilities are presented.

[Contact: William E. Anderson, (301) 975-2432]

Fenimore, C., and Martzloff, F.D., Validating Surge Test Standards by Field Experience: High-Energy Tests and Varistor Performance, Conference Record of the 1990 IEEE Industry Applications Society Annual Meeting, Seattle, Washington, October 7-12,
1990, pp. 1968-1974 (1990).

New, high-energy surge tests are emerging in IEEE and IEC standards. Field experience offers a valuable criterion for validating or invalidating proposed standards. A proposal under consideration by the IEC involves so much energy that a varistor of the voltage rating commonly used in protecting load equipment, if subjected to this test, would almost certainly fail. Yet, reported varistor failure rates do not reflect such a situation. Thus, a re-examination of the premises that led to the proposed test specifications appears necessary. Proposals for high-energy tests as additional waveforms in the new version of IEEE C62.41, on the other hand, lead to current and energy levels that do not place typical varistors in immediate jeopardy. Thus, they appear more consistent with field experience.

[Contact: Charles Fenimore, (301) 975-2428]

FitzPatrick, G.J., and Lagnese, J.E., Determination of Kerr Cell Parameters with Comparative Digitizer Measurements, Proceedings of the IEEE International Symposium on Digital Techniques in HighVoltage Measurements, Toronto, Ontario, Canada, October 28-30, 1991, pp. 5-29 to 5-33 (1991).

Kerr cell measurement systems consist of polarimetric devices that modulate a beam of light according to the voltage applied to them. The output consists of a series of intensity oscillations or "fringes." Calibration of Kerr cells have been performed in the past through simultaneous measurements of the Kerr cell and voltage divider outputs using analog oscilloscopes. The evaluation of the Kerr cell constant was then made by comparison of the peak voltages as measured by the two systems. Digitized data are ideal for the application of curve-fitting techniques and are now used for the evaluation of the Kerr parameters (or alternatively, for the determination of the ratio of the voltage divider). The fitting techniques utilize the entire waveforms rather than comparing them at peak voltage only. This paper describes the application of curve-fitting techniques to digitized waveforms for the evaluation of the Kerr cell constants. The results for Kerr cells used to cover the range from $10 \mathrm{kV}$ to 300 $\mathrm{kV}$ are presented. Cell constants for the same cell geometry but with different Kerr liquids are also reported. The uncertainties of the evaluated Kerr cell parameters and their dependence on fringe number are discussed. The effects on the evaluated cell 
constants produced by segmenting the digitized Kerr waveforms are also examined.

[Contact: Gerald J. FitzPatrick, (301) 975-2737]

Martzloff, F.D., A Standard for the 90s: IEEE C62.41

Surges Ahead [original title: IEEE Guide on Surge Voltages Upgraded to Recommended Practice], Compliance Engineering, Vol. 8, No. 5, pp. 27-ff (Fall 1991).

After ten years of use as a guide, a revision has been completed and published as an IEEE Recommended Practice: Surge Voltages in Low-Voltage AC Power Circuits. This article appears in a trade magazine circulated to writers, users, and enforcers of standards on electromagnetic compatibility in order to give them a preview of the forthcoming IEEE document.

[Contact: Francois D. Martzloff, (301) 975-2409]

Martzloff, F.D., and Lai, J.S., Cascading SurgeProtective Devices: Coordination Versus the IEC 664 Staircase, Proceedings of the First International Conference on Power Quality: End-Use Applications and Perspectives, Gif-sur-Yvette, Paris, France, October 15-18, 1991, pp. 191-198.

Cascading two or more surge-protective devices located, respectively, at the service entrance of a building and near the sensitive equipment is intended to ensure that each device shares the surge stress in a manner commensurate with its rating to achieve reliable protection of equipment against the surges impinging from the utility supply, as well as internally generated surges. However, depending upon the relative clamping voltages of the two devices, their separation distance, and the waveform of the impinging surge, coordination may or may not be effective. The paper reports computations confirmed by measurements of the energy deposited in the devices for combination of these three parameters.

[Contact: Francois D. Martzloff, (301) 975-2409]

Martzloff, F.D., and Mendes, A., Standards: Transitional Aspects, Proceedings of the First International Conference on Power Quality: End-Use Applications and Perspectives, Gif-sur-Yvette, Paris, France, October 15-18, 1991, pp. 31-34.

Mass production of electrical and electronic equipment for the world market requires a system of standards of worldwide applicability. The develop- ment of such standards is a complex task, involving various national and international organizations. This paper presents a review of the standards-writing process, in particular, the area of power quality.

[Contact: Francois D. Martzloff, (301) 975-2409]

McComb, T.R., and Lagnese, J.E., Calculating the Parameters of Full Lightning Impulses Using Model-Based Curve Fitting, IEEE Transactions on Power Delivery, Vol. 6, No. 4, pp. 1386-1394 (October 1991).

A brief review is presented of the techniques used for the evaluation of the parameters of high-voltage impulses and the problems encountered. The determination of the best smooth curve through oscillations on a high-voltage impulse is the major problem limiting the automatic processing of digital records of impulses. Nonlinear regression, based on simple models, is applied to the analysis of simulated and experimental data of full lightning impulses. Results of model fitting to four different groups of impulses are presented and compared with some other methods. Plans for the extension of this work are outlined. [Contact: Terry R. McComb, (301) 975-3954]

Misakian, M., In Vitro Exposure Parameters with Linearly and Circularly Polarized ELF Magnetic Fields, Bioelectromagnetics, Vol. 12, No. 6, pp. 377 381 (October 1991).

A comparison is made of induced current densities, electric fields, and rate of energy deposition during invitro studies with linearly and circularly polarized extremely low frequency (ELF) magnetic fields.

[Contact: Martin Misakian, (301) 975-2426]

Olthoff, J.K., Van Brunt, R.J., Wang, H-X., Moore, J.H., and Tossell, J.A., Total Cross Sections for Electron Scattering and Attachment for $\mathrm{SF}_{6}$ and Its Electrical-Discharge By-Products [original title: Absolute Total Electron Scattering and Dissociative Attachment Cross Sections of By-Products from Electrical Discharges in $\mathrm{SF}_{6}$ ], Gaseous Dielectrics VI, L. G. Christophorou and I. Sauers, Eds. (Plenum Press, New York, 1991), pp. 19-25. [Proceedings of the Sixth International Symposium on Gaseous Dielectrics, Knoxville, Tennessee, September 23-27, 1990.]

Using an electron transmission spectrometer, the 
absolute total dissociative attachment cross sections of $\mathrm{SF}_{6}$ and of its decomposition products have been measured as a function of electron energy over the range of $0.2 \mathrm{eV}$ to $5.0 \mathrm{eV}$, and absolute total electron scattering cross sections have been measured from 0.2 $\mathrm{eV}$ to $12 \mathrm{eV}$. These results are presented along with previous data where available.

[Contact: James K. Olthoff, (301) 975-2431]

Sauers, I., Harman, G., Olthoff, J.K., and Van Brunt, R.J., $\mathrm{S}_{2} \mathrm{~F}_{10}$ Formation by Electrical Discharges in $\mathrm{SF}_{6}$ : Comparison of Spark and Corona, Gaseous Dielectrics VI, L. G. Christophorou and I. Sauers, Eds. (Plenum Press, New York, 1991), pp. 553-561. [Proceedings of the Sixth International Symposium on Gaseous Dielectrics, Knoxville, Tennessee, September 23-27, 1990.]

Among the $\mathrm{SF}_{6}$ by-products of electrical discharges that have been investigated, $\mathrm{S}_{2} \mathrm{~F}_{10}$ is probably the least understood (physical, chemical, and biological properties) and the most toxic. Its production in electrical discharges has been controversial since the presence of this chemical has been reported by only a few groups. We report on the yields of $\mathrm{S}_{2} \mathrm{~F}_{10}$ in two types of discharges: spark and corona. The $\mathrm{S}_{2} \mathrm{~F}_{10}$ yields for corona and spark were $2.4 \mu \mathrm{mol} / \mathrm{C}$ and 0.04 to $0.37 \mathrm{nmol} / \mathrm{J}$, respectively, for experiments where the water content was low. For both types of discharges, we have found that $\mathrm{S}_{2} \mathrm{~F}_{10}$ formation is dependent on the presence of moisture. For corona discharges, model calculations based on known sulfur-fluorine chemistry are shown to yield reasonable agreement with experimental data. We show that $\mathrm{S}_{2} \mathrm{~F}_{10}$ was formed in electrical discharges expected to be found in compressed-gas insulated equipment, and address such factors as effects of moisture and surface conditions.

[Contact: James K. Olthoff, (301) 975-2425]

Turgel, R.S., Electricity in the Year 2000, Proceedings of the IEEE Technical Activities Board (TAB) Symposium, Mexico City, Mexico, October 3, 1991, pp. 1-5.

Demand for electricity is predicted to rise at a rate higher than that of the creation of new generating capacity. Projections suggest that a shortfall of 100 million kilowatts may be reached by the year 2000 . Various measures to alleviate the projected shortfall are discussed.
[Contact: Raymond S. Turgel, (301) 975-2420]

Van Brunt, R.J., $\mathbf{S}_{\mathbf{2}} \mathrm{F}_{10}$ : A Clarification, Electrical Review, Vol. 224, No. 22, p. 10 (November 15, 1991).

"SF 6 product '3,000 times deadlier than phosgene"" (Electrical Review, 20 September-3 October) reports, among other topics, results obtained by researchers at the National Institute of Standards and Technology. This letter to the editor addresses some misstatements that occur in the article, referring to the NIST work. [Contact: Richard J. Van Brunt, (301) 975-2425]

Van Brunt, R.J., Stochastic Properties of PartialDischarge Phenomena, IEEE Transactions on Electrical Insulation, Vol. 26, No. 5, pp. 902-948 (October 1991).

Prebreakdown pulsating partial-discharge (P-D) phenomena that occur in dielectric media are inherently complex stochastic processes that exhibit significant statistical variability in such properties as pulse amplitude, shape, and time of occurrence. Previously published work concerned with the theory and measurement of the stochastic behavior of PDs is reviewed. The types of P-D phenomena considered in this review include ac- and dc-generated electron avalanches, pulsating positive and negative corona in gases, and partial discharges that occur in liquid media and in the presence of solid dielectric surfaces. The basic physical mechanism distributions for pulse occurrence times and pulse amplitudes are discussed. Consideration is also given to special problems associated with the measurement and interpretation of data on the various statistical properties of P-D phenomena.

[Contact: Richard J. Van Brunt, (301) 975-2425]

Van Brunt, R.J., and Cernyar, E.W., Influence of Phase-to-Phase Memory Propagation on the Stochastic Behavior of AC-Generated Partial Discharges, 1991 Annual Report, Conference on Electrical Insulation and Dielectric Phenomena (CEIDP), Knoxville, Tennessee, October 20-23, 1991, pp. 589596.

From measurements of phase-restricted conditional partial-discharge amplitude and phase-of-occurrence distributions performed for the first time, it has been 
possible to observe the influence of phase-to-phase memory propagation on the stochastic behavior of partial discharges generated by applying an ac voltage to a point electrode in contact with a solid dielectric surface. "Memory" associated with charge deposited on the dielectric surface by preceding discharge events is found to have a significant effect in determining the most probable phase-of-occurrence and amplitudes of subsequent partial-discharge pulses. It is found, for example, that the larger amount of charge deposited during partial-discharge activity on one-half cycle, the sooner will be the time (or phase) of occurrence of the partial discharges on the next half cycle. The observed memory effect is expected from consideration of the surface charging dynamics and must be considered in any attempt to interpret results of phase-resolved partial-discharge measurements.

[Contact: Richard J. Van Brunt, (301) 975-2425]

Van Brunt, R.J., and Kulkarni, S., Influence of Memory on the Statistics of Pulsating Corona, Gaseous Dielectrics VI, L. G. Christophorou and I. Sauers, Eds. (Plenum Press, New York, 1991), pp. 383-389. [Proceedings of the Sixth International Symposium on Gaseous Dielectrics, Knoxville, Tennessee, September 23-27, 1990.]

In order to develop a theory that accounts for observed pulse-time separation and pulse-amplitude distributions for pulsating corona discharges in gases, it is necessary to consider the effects of residuals from prior discharge pulses, such as ion space charge and metastables, on the development of subsequent pulses. Such "memory effects" are shown here to be significant in controlling the statistics of Trichel-pulse corona in electro-negative gases. The memory effects are quantitatively assessed from a direct measurement of a set of conditional pulse-amplitude and pulsetime-separation distributions. The effectiveness of this method in providing a more complete description and better understanding of the stochastic behavior of corona is illustrated here for the case of self-sustained Trichel pulse in a neon-oxygen gas mixture. The amplitude and time of initiation of any discharge pulse is found to be strongly dependent on the amplitude of the previous pulse, as well as on the time that has elapsed since that pulse occurred. Memory is found to extend back beyond the most recent pulse so that the process is distinctly non-Markovian.

[Contact: Richard J. Van Brunt, (301) 975-2425]
Magnetic Materials and Measurements

\section{Released for Publication}

Day, G.W., Deeter, M.N., and Rose, A.H., Faraday Effect Sensors: A Review of Recent Progress, to be published in the Proceedings of the International Conference on Optical Fiber Sensors, Wuham, China, October 9-11, 1991.

The last few years have seen dramatic progress in the development of Faraday effect sensors for measuring both magnetic fields and electric current. In the case of magnetic field sensors, the most significant advances have resulted from an investigation of new materials, especially the ferrimagnetic iron garnets. Minimum detectable magnetic fields have been reduced by several orders of magnitude to about 100 $\mathrm{pT} / \sqrt{ } \mathrm{Hz}$ at $500 \mathrm{~Hz}$, and bandwidths of hundreds of megahertz have been obtained. In the case of electric current sensors, new methods of avoiding the effects of linear birefringence in optical fiber have resulted in much smaller and more stable sensors. One of those approaches, annealing of the fiber to reduce birefringence, has yielded sensors with temperature stabilities near that of the material limit, $<10^{-4} / \mathrm{K}$. These topics and other developments that have occurred since a previous summary [Proc. SPIE 985 138-150 (1988)] are discussed in this review.

[Contact: Gordon W. Day, (303) 497-5204]

Deeter, M.N., and Williams, P.A., Magnetooptic Characterization of Iron Garnet Crystals Using Photoelastic Modulation, to be published in the Conference Digest, Intermag 92, St. Louis, Missouri, April 13-16, 1992.

We describe an experimental technique in which a photoelastic modulator is used to simultaneously measure Faraday rotation and depolarization in magnetooptic materials. We report Faraday rotation and depolarization data for the glass SF-57 and for yttrium iron garnet (YIG). The YIG sample exhibits substantial depolarization in small applied magnetic fields.

[Contact: Merritt N. Deeter, (303) 497-5400]

Fickett, F.R., Low Temperatu re Magnetic Behavior of "Nonmagnetic" Materials.

The magnetically sensitive designs for many super- 
conductor systems, ranging from large magnets to thin film devices, require a knowledge of the magnetic properties of a wide range of materials. Commercial "nonmagnetic" materials may show bizarre magnetic behavior as a function of temperature, changing from paramagnetic to diamagnetic, or vice versa, as the temperature is lowered and sometimes even becoming ferromagnetic. In metallic alloys, whether and when these effects occur is often determined by the exact composition of the alloy, which is frequently correlated with its age. Furthermore, nonmetallic materials may have strong magnetic signatures which arise from magnetic impurities, such as inclusions of magnetite in the glass fibers of fiberglass epoxies. Here, we summarize results of magnetic susceptibility measurements on a number of metallic alloys and some nonmetallic materials used in cryogenic applications. The data suggest that care should be taken in the use of many of these common materials, especially in the construction of sensitive magnetometer systems.

[Contact: Fred R. Fickett, (303) 497-3785]

Geyer, R.G., Baker-Jarvis, J.R., Janezic, M.D., and Domich, P.D., Spectral Characterization of Ferrites for Use as Magnetic Reference Materials, to be published in the Conference Record, Conference on Precision Electromagnetic Measurements (CPEM '92), Paris, France, June 9-12, 1992.

Broadband complex initial permeability and permittivity spectra of various ferrites are examined in order to assess their suitability for use as magnetic reference materials. These constant-temperature spectral measurements are made with coaxial transmission line on sintered poly-crystalline spinels and garnets of varying compositions, as well as on loaded polymers. Laboratory measurement uncertainties in both dielectric and magnetic properties are evaluated. An example of measured complex permeability and permittivity spectra of a polycrystalline $\mathrm{Ni}-\mathrm{Zn}$ ferrite possessing high initial permeability with relatively low dielectric loss is given at frequencies from $300 \mathrm{kHz}$ to $10 \mathrm{GHz}$. A strong single resonance behavior is apparent at frequencies between $800 \mathrm{MHz}$ and 2 $\mathrm{GHz}$.

[Contact: Richard G. Geyer, (303) 497-5852]

Goldfarb, R.B., Lelental, M., and Thompson, C.A., Alternating-Field Susceptometry and Magnetic Susceptibility of Superconductors, to be published as NISTIR 3977.
This review critically analyzes current practice in the design, calibration, sensitivity determination, and operation of alternating-field susceptometers, and examines applications in magnetic susceptibility measurements of superconductors. Critical parameters of the intrinsic and coupling components of granular superconductors may be deduced from magnetic susceptibility measurements. The onset of intrinsic diamagnetism corresponds to the initial decrease in electrical resistivity upon cooling, but the onset of intergranular coupling coincides with the temperature for zero resistivity. The lower critical field may be determined by the field at which the imaginary part of susceptibility increases from zero. Unusual features in the susceptibility of superconductor films, such as a magnetic moment that is independent of film thickness and the variation of susceptibility with angle, are related to demagnetization. Demagnetizing factors of superconductor cylinders are significantly different from those commonly tabulated for materials with small susceptibilities. Rules for the susceptibility of mixtures with specific demagnetizing factors are used to estimate the volume fraction of superconducting grains in sintered materials. Common misunderstandings of the Meissner effect, magnetic units, and formula conversions are discussed. There is a comprehensive summary of critical-state formulas for slabs and cylinders, including new equations for complex susceptibility in large alternating fields. Limitations on the use of the critical-state model for deducing critical current density are listed, and the meaning of the imaginary part of susceptibility is considered.

[Contact: Ronald B. Goldfarb, (303) 497-3650]

Misakian, M., and IEEE Magnetic Fields Task Force (Members of the AC Fields Working Group in the IEEE Power Engineering Society), A Protocol for Spot Measurements of Residential Power Frequency Magnetic Fields.

This paper describes a simple protocol for measuring power frequency magnetic fields in residences. The protocol should not be interpreted as an IEEE standard, but if followed on a trial basis will provide for a degree of procedural uniformity which currently does not exist. The measurement procedures are simple and are not intended to characterize the temporal and spatial variability of magnetic field levels in residences. The protocol contains a structured component during which measurements are performed 
at specific locations and an unstructured component during which measurements are performed at locations requested by the occupants. The protocol requires personnel performing the measurements to explain the metrology-related limitations associated with the measurement results. This paper also discusses terminology related to power frequency magnetic fields and their measurement, the meaning of measurement accuracy and variability within the context of residential magnetic field measurements, and examples of measurement approaches that characterize the variability of magnetic field levels. [Contact: Martin Misakian, (301) 975-2426]

Nogues, J., Moreland, J., Rao, K.V., Inoue, A., and Suzuki, K., Microstructure of Amorphous and Nanocrystalline Fe-Zr-B-Cu Ribbons: A Scanning Tunneling Microscope Study.

The surface microstructures of both amorphous and annealed $\mathrm{Fe}_{86} \mathrm{Zr}_{7} \mathrm{~B}_{6} \mathrm{Cu}_{1}$ alloys have been studied by means of scanning tunneling microscopy. The amorphous sample is found to have a smooth, almost featureless surface. On the other hand, the annealed sample has ellipsoidal shaped cluster $(\approx 300 \times 150$ $\mathrm{nm}$ ) aligned along the casting direction and consisting of randomly oriented nanocrystallites of about $10 \mathrm{~nm}$ in diameter which is consistent with TEM results. The nanocrystallites themselves have a smooth morphology, rather than a grainy rough surface. The small size of the nanocrystals is shown to be the cause of the improved soft magnetic properties in the annealed alloy.

[Contact: John Moreland, (303) 497-3641]

Wolfe, R., Gyorgy, E.M., Lieberman, R.A., Fratello, V.J., Licht, S.J., Deeter, M.N., and Day, G.W., High Frequency Magnetic Field Sensors Based on the Faraday Effect in Garnet Thick Films.

The Faraday effect in the thick epitaxial films of magnetic garnets of the type used in magneto-optic isolators can be used as the basis for a fiber-optic magnetic field sensor. These films have uniaxial anisotropy perpendicular to the surface, and they contain bismuth to enhance the Faraday rotation. The typical magnetic domain pattern of meandering stripes changes in response to an applied field perpendicular to the film, and this changes the polarization of infrared light propagating perpendicular to the film. Theory and experiment show that the speed of opera- tion is limited by relaxation or resonance effects to upper frequencies between $10^{6}$ and $10^{9} \mathrm{~Hz}$. Maximum sensitivity requires low magnetic moment and large thickness in conflict with the requirements for high speed.

[Contact: Merritt N. Deeter, (303) 497-5400]

\section{Recently Published}

Chen, D-X., Brug, J.A., and Goldfarb, R.B., Demagnetizing Factors for Cylinders, IEEE Transactions on Magnetics, Vol. 27, No. 4, pp. 3601-3619 (July 1991).

We have calculated fluxmetric and magnetometric demagnetizing factors $\mathrm{N}_{\mathrm{f}}$ and $\mathrm{N}_{\mathrm{m}}$ for cylinders as functions of susceptibility $\chi$ and the ratio of length to diameter $\gamma$. For $\chi=0$, applicable to weakly magnetic or saturated ferromagnetic materials, $\mathrm{N}_{\mathrm{f}}$ and $\mathrm{N}_{\mathrm{m}}$ are calculated using a formula for the mutual inductance of concentric coaxial thin solenoids. $\mathrm{N}_{\mathrm{f}}$ for $-1 \leq \chi<$ $\infty$ and $\mathrm{N}_{\mathrm{m}}$ for $\chi \rightarrow \infty$ when $\gamma \geq 10$ are calculated using a one-dimensional model. For $1 \leq \gamma \leq 10$, an important range for magnetometer measurements, $\mathrm{N}_{\mathrm{m}}$ for both $\chi \rightarrow \infty$ and $\chi<0$ are obtained by extrapolation from data at larger $\gamma$. The case $\chi<0$ is applicable to conductors and superconductors. General rules for demagnetizing factors are discussed.

[Contact: Ronald B. Goldfarb, (303) 497-3650]

Moreland, J., and Rice, P., Imaging Magnetic Bit Patterns Using a Scanning Tunneling Microscope with a Flexible Tip, Proceedings of the Materials Research Society Symposium, Anaheim, California, April 30-May 3, 1991, Vol. 232, pp. 141-146.

Tunneling stabilized magnetic force microscopy (TSMFM) is a variant of scanning tunneling microscopy (STM) where the usual rigid STM tip is replaced with a flexible magnetic tip. This method contrasts with other magnetic force microscopes based on optical detection of cantilever deflection due to magnetic forces. Instead, the position of the flexible tunneling tip depends on both topography and magnetic forces acting on the end of the tip. The $z$-motion of the piezoelectric translator flexes the tip to balance the magnetic force so that the end of the tip remains a fixed tunneling distance from the sample surface. We present TSMFM images showing the recorded bit patterns of hard disk, floppy disk, and tape surfaces. The images were taken in air using 
free-standing, thin-film (about $1-\mu \mathrm{m}$-thick) Fe and $\mathrm{Ni}$ flexible STM tips. The image resolution of TSMFM is routinely submicrometer. We conclude that this simple modification of STM will be a valuable diagnostic tool in the magnetic recording industry.

[Contact: John Moreland, (303) 497-3641]

Superconductors

\section{Released for Publication}

Cutro, J.A., Orlando, T.P., van Dover, R.B., Schneemeyer, L.F., White, A.E., Gyorgy, E.M., Waszczak, J.V., Felder, R.J., and Rudman, D.A., Increased Pinning Energies and Critical Current Densities in Heavy-Ion-Irradiated $\mathrm{Bi}_{2} \mathrm{Sr}_{2} \mathrm{CaCu}_{2} \mathrm{O}_{8}$ Single Crystals.

We report a significant increase in the pinning energy of vortices in single-crystal $\mathrm{Bi}_{2} \mathrm{Sr}_{2} \mathrm{CaCu}_{2} \mathrm{O}_{8}$ when irradiated with heavy ions such as $\mathrm{Ar}^{+}$. This is in contrast with the results of light ion $\left(\mathrm{H}^{+}, \mathrm{He}^{+}\right)$ irradiations which give pinning energies comparable with those of unirradiated crystals. The stronger pinning is attributed to defects larger than point defects, e.g., clusters or amorphized regions. As a result of higher pinning energies, critical currents persist at markedly higher temperatures and fields. [Contact: David A. Rudman, (303) 497-5081]

\section{Ekin, J.W., Mechanical and Magnetic-Field-Angle}

Properties of High- $\mathrm{T}_{c}$ Superconductors, to be published in the Proceedings of the 7th U.S.-Japan Workshop on High-Field Superconductivity Materials, Wires, and Conductors and Standard Procedures for HTSC Wires Testing, Fukuoka, Japan, October 18-24, 1991.

Electromechanical properties have been measured for Ag-sheathed $\mathrm{Bi}_{2} \mathrm{Sr}_{2} \mathrm{Ca}_{1} \mathrm{Cu}_{2} \mathrm{O}_{8+\mathrm{x}}$ superconductors at high magnetic fields up to $25 \mathrm{~T}$. A melt-processed powder-in-tube $\mathrm{Bi}_{2} \mathrm{Sr}_{2} \mathrm{Ca}_{1} \mathrm{Cu}_{2} \mathrm{O}_{8+\mathrm{x}}$ conductor was measured to have an irreversible strain of $0.2 \%$ and a $50 \%$ critical-current-degradation strain of $0.36 \%$. Measurements of a discontinuous filament meltprocessed Ag-sheathed $\mathrm{Bi}_{2} \mathrm{Sr}_{2} \mathrm{Ca}_{1} \mathrm{Cu}_{2} \mathrm{O}_{8+x}$ superconductor show an irreversible strain of $0.6 \%$ and a $50 \%$ degradation strain of about $1 \%$. These strain damage thresholds are about an order of magnitude higher than for high-temperature superconductors made by bulk sintering and are reaching practical values for magnet design. High magnetic field measurements of $\mathrm{J}_{\mathrm{c}}$ as a function of the angle of $\mathbf{B}$ with respect to the C-axis are also reported for an oriented-grained $\mathrm{YBa}_{2} \mathrm{Cu}_{3} \mathrm{O}_{7}$ sample. In contrast to earlier thin-film results at lower fields $(<3 \mathrm{~T})$, the measurements reported here in high fields reveal a $\mathrm{J}_{\mathrm{c}}$-versus-angle curve with a head-and-shoulders shape, consisting of a sharp peak ("head") $<5^{\circ}$ wide when $\mathbf{B}$ is parallel to the $\mathrm{CuO}_{2}$ planes, with a wide $\left(30^{\circ}\right.$ at $9 \mathrm{~T}$, for example) shoulder region on either side of the peak where the transport $J_{c}$ remains high and constant. At still higher angles, however, the transport $\mathrm{J}_{\mathrm{c}}$ decreases sharply, giving rise to the concept of a critical field angle for application design.

[Contact: John W. Ekin, (303) 497-5448]

Ekin, J.W., and Bray, S.L., Transverse Stress and Crossover Effects in $\mathrm{Nb}_{3} \mathrm{Sn}$, to be published in the Proceedings of the 7th U.S.-Japan Workshop on High-Field Superconductivity Materials, Wires, and Conductors and Standard Procedures for HTSC Wires Testing, Fukuoka, Japan, October 18-24, 1991.

The superconducting wires in an energized magnet coil are subjected to mechanical stresses caused by the Lorentz force. Previous measurements have shown that either axial tensile stress or transverse compressive stress, the two dominant stresses on the wire, can cause substantial degradation in the superconductor's critical current. The previous transverse stress measurements were made with uniformly applied stress; however, many superconductor applications employ cables where the strands experience stress concentrations at the points where they cross one another. For this study, a single stress concentration point was simulated by applying transverse stress to two $\mathrm{Nb}_{3} \mathrm{Sn}$ wires, which were crossed over each another at a specified angle while measuring the critical current of one of the wires at magnetic fields up to $9 \mathrm{~T}$. A comparison between the cross-over-transverse-stress measurements and the uniform-transverse-stress measurements shows a comparable degradation in critical current at equivalent stresses; however, this implies that the critical-current degradation at equivalent loads will be greater for the cross-over situation due to the reduced area. Although this result may facilitate the calculation of critical-current degradation in cables from the existing uniform-transverse-stress data, it also suggests that these conductors may be more sensitive to transverse loads than conductors 
that have a more uniform stress distribution. [Contact: John W. Ekin, (303) 497-5448]

Goldfarb, R.B., Lelental, M., and Thompson, C.A., Alternating-Field Susceptometry and Magnetic Susceptibility of Superconductors, to be published as NISTIR 3977.

This review critically analyzes current practice in the design, calibration, sensitivity determination, and operation of alternating-field susceptometers, and examines applications in magnetic susceptibility measurements of superconductors. Critical parameters of the intrinsic and coupling components of granular superconductors may be deduced from magnetic susceptibility measurements. The onset of intrinsic diamagnetism corresponds to the initial decrease in electrical resistivity upon cooling, but the onset of intergranular coupling coincides with the temperature for zero resistivity. The lower critical field may be determined by the field at which the imaginary part of susceptibility increases from zero. Unusual features in the susceptibility of superconductor films, such as a magnetic moment that is independent of film thickness and the variation of susceptibility with angle, are related to demagnetization. Demagnetizing factors of superconductor cylinders are significantly different from those commonly tabulated for materials with small susceptibilities. Rules for the susceptibility of mixtures with specific demagnetizing factors are used to estimate the volume fraction of superconducting grains in sintered materials. Common misunderstandings of the Meissner effect, magnetic units, and formula conversions are discussed. There is a comprehensive summary of critical-state formulas for slabs and cylinders, including new equations for complex susceptibility in large alternating fields. Limitations on the use of the critical-state model for deducing critical current density are listed, and the meaning of the imaginary part of susceptibility is considered.

[Contact: Ronald B. Goldfarb, (303) 497-3650]

\section{Goodrich, L.F., and Srivastava, A.N., Superconductor Critical Current Simulation and Data Acquisition.}

The superconductor simulator is an electronic circuit that emulates the extremely nonlinear voltage-current characteristic (the basis of a critical-current measurement) of a superconductor along with its other major electrical properties. We designed three different types of simulators: the passive, temperature-controlled passive, and active simulators. These simulators are high-precision instruments, and are thus useful for establishing the integrity of part of a superconductor measurement system. They could significantly benefit superconductor measurement applications that require high-precision quality assurance.

We have also designed software to control our computer-driven data acquisition and analysis system. It uses various algorithms to efficiently characterize the conductor's electrical properties, and generates summaries of the acquisition and analysis phase of the measurement along with plots of relevant data. The software and the superconductor simulator serve as diagnostic tools for determining sources of systematic and random errors in our measurement systems.

[Contact: Loren F. Goodrich, (303) 497-3143]

Goodrich, L.F., and Srivastava, A.N., Trends in Superconductor Critical-Current Measurement Technology in the USA, to be published in the Proceedings of the 4th Annual U.S./Japan Workshop on High Temperature Superconductivity, Gaithersburg, Maryland, November 25-26, 1991.

This paper indicates trends in superconductor measurement technology in the United States, and discusses available methods to reduce measurement uncertainty and imprecision. The results of interlaboratory comparisons of critical-current measurements have indicated that a detailed sample test procedure is essential to reduce interlaboratory measurement variation. High-temperature superconductors are particularly susceptible to degradation with time, mounting, and use. These factors contribute to the overall uncertainty in the measurement, and play a similar role in the measurement uncertainty as do random processes. A standard reference material such as SRM-1457 or a superconductor simulator can greatly aid in identifying sources of measurement variation. Although high-temperature and low-temperature superconductor technologies are in different states of maturity, their respective uncertainties in critical current may be reduced using a detailed sample test procedure.

[Contact: Loren F. Goodrich, (303) 497-3143]

Kucera, J.T., Rubin, L.M., Uwai, K., Perkins, J.D., Graybeal, J.M., Orlando, T.P., Vander Sande, J.B., 
Roshko, A., and Moreland, J., Fabrication of Nanometer Smooth $\mathrm{Bi}_{2} \mathrm{Sr}_{2} \mathrm{CaCu}_{2} \mathrm{O}_{8+\delta}$ Films by Reactive Co-Sputtering from Elemental Targets with Pure Ozone.

We describe the fabrication of superconducting $\mathrm{Bi}_{2} \mathrm{Sr}_{2} \mathrm{CaCu}_{2} \mathrm{O}_{8+\delta}$ thin films having root-mean-square surface roughness of less than $5 \mathrm{~nm}$ as determined by scanning tunneling microscopy. Films are deposited "in situ" by rf and dc triode magnetron sputtering from elemental metallic targets in the presence of pure ozone. As deposited, these films have transition temperatures as high as $68 \mathrm{~K}$, and zero-field critical current densities exceed $10^{6} \mathrm{~A} / \mathrm{cm}^{2}$ at $4.2 \mathrm{~K}$. The transition temperatures can be increased to $80 \mathrm{~K}$ by post-deposition annealing with only a slight increase in surface roughness.

[Contact: Alexana Roshko, (303) 497-5420]

\section{Recently Published}

\section{Ekin, J.W., Effect of Cable and Strand Twist-Pitch Coincidence on the Critical Current of Flat, Core- less Superconductor Cables, Applied Physics Letters, Vol. 59, No. 20, pp. 2615-2617 (11 November 1991).}

We present data which indicate that a very simple technique of enhancing the critical current in flat, coreless superconductor cables is to match the cable twist pitch with the strand twist pitch. In this manner, the same group of filaments within each strand are degraded at each successive bend at the cable edges. This coincident-twist method minimizes current transfer among filaments, enhances the slope of the voltage-current characteristic, consistently improves the critical current by about $10 \%$ in these tests, and is easy to apply.

[Contact: John W. Ekin, (303) 497-5448]

Goodrich, L.F., Critical Current Measurement on High $T_{c}$ Superconductors, Report of the Third Annual U.S.-Japan Workshop on Semiconductors, Buffalo, New York, September 20-21, 1990, pp. 6468 (1990).

A passive voltage-current (V-I) simulator has been developed and tested using conventional direct current (dc), lock-in amplifier and pulse current methods. The simulator was designed to generate the extremely nonlinear V-I characteristic of a superconductor. It is intended to be used to test various components of the measurement system, such as instrumentation, measurement method, and data analysis software, to determine the transport critical current $\left(I_{c}\right)$ or critical current density $\left(J_{c}\right)$ of a superconductor. Comparison of the pulse current and the lock-in amplifier methods with the conventional direct current method on the passive simulator is presented. Also, preliminary comparisons of $\mathrm{dc}$ and pulse methods using a thin-film YBCO sample are given.

[Contact: Loren F. Goodrich, (303) 497-3143]

Goodrich, L.F., Srivastava, A.N., and Stauffer, T.C., Simulators of Superconductor Critical Current: Design, Characteristics, and Applications, Journal of Research of the National Institute of Standards and Technology, Vol. 96, No. 6, pp. 703-724 (NovemberDecember 1991).

The superconductor simulator is an electronic circuit that emulates the extremely nonlinear voltage-current characteristic (the basis of a critical-current measurement) of a superconductor along with its other major electrical properties. Three different types of simulators have been constructed: the passive, active, and hybrid simulator. The passive simulator has the fewest circuit components and offers the least amount of versatility, while the active and hybrid simulators offer more versatility and consequently have more components. Design, characteristics, and applications of the superconductor simulator along with a summary of features are presented. These simulators are high-precision instruments, and are thus useful for establishing the integrity of part of a superconductor measurement system. They are potentially useful for testing the measurement method and data acquisition and analysis routines. The 50-A simulator provides critical-current precision of $0.1 \%$ at a $1-\mu \mathrm{V}$ signal. This is significantly higher than the precision of a superconducting standard reference material. The superconductor simulator could significantly benefit superconductor measurement applications that require high-precision quality assurance.

[Contact: Loren F. Goodrich, (303) 497-3143]

Ishida, T., Goldfarb, R.B., Okayasu, S., and Kazumata, Y., Static and Nonlinear Complex Susceptibility of $\mathrm{YBa}_{2} \mathrm{Cu}_{3} \mathrm{O}_{7}$, Physica C, Vol. 185189 (Elsevier Science Publishers B.V., North-Holland, 1991), pp. 2515-2516.

We have investigated the harmonic susceptibility $\chi_{\mathrm{n}}=$ 
$\chi_{\mathrm{n}^{\prime}}-\mathrm{i} \chi_{\mathrm{n}^{\prime \prime}}(\mathrm{n}=1,2,3, \ldots)$ of sintered $\mathrm{YBa}_{2} \mathrm{Cu}_{3} \mathrm{O}_{7}$ in the field of $\mathrm{H}_{\mathrm{dc}}+\mathrm{H}_{\mathrm{ac}} \sin \omega \mathrm{t}$. $\chi_{1^{\prime}}$ and $\chi_{1^{\prime \prime}}$ as functions of temperature depend on both $\mathrm{H}_{\mathrm{ac}}$ and $\mathrm{H}_{\mathrm{dc}}$. Both even and odd $\chi_{\mathrm{n}}{ }^{\prime}$ s were detected for nonzero $\mathrm{H}_{\mathrm{dc}}$, but only odd harmonics were observed for zero $\mathrm{H}_{\mathrm{dc}}$. At constant temperature, $\chi_{\mathrm{n}}$ is an even function of $\mathrm{H}_{\mathrm{dc}}$ for $\mathrm{n}=$ odd, while $\chi_{\mathrm{n}}$ is an odd function of $\mathrm{H}_{\mathrm{dc}}$ for $\mathrm{n}$ = even. We compared experimental features with the prediction of the critical state model. For comparison, the static susceptibility $\chi_{\mathrm{dc}}$ of $\mathrm{YBa}_{2} \mathrm{Cu}_{3} \mathrm{O}_{7}$ was examined as a function of temperature. The $\chi_{\mathrm{dc}}(\mathrm{T})$ for the sample cooled in small fields is unusual; a negative peak occurs near $T_{c}$, which can be explained by intragranular flux depinning upon warming.

[Contact: Ronald B. Goldfarb, (303) 497-3650]

Loughran, R.J., and Goldfarb, R.B., Anisotropic Weak-Link Properties and Intergranular Lower Critical Field of Grain-Aligned $\mathrm{YBa}_{2} \mathrm{Cu}_{3} \mathrm{O}_{\mathrm{x}}$, Physica C, Vol. 181 (Elsevier Science Publishers B.V., North-Holland, 1991), pp. 138-142.

We studied the weak-link nature of sintered, grainaligned $\mathrm{YBa}_{2} \mathrm{Cu}_{3} \mathrm{O}_{\mathrm{x}}$ using dc magnetization and ac susceptibility. The sample was highly anisotropic for fields applied perpendicular and parallel to the grains' a,b planes. The bulk demagnetizing factor was the same for the two field directions. For fields applied perpendicular to the a,b planes, the magnetization curves show small intergranular coupling losses, and the susceptibility curves show sharp coupling transitions. From ac susceptibility measurements in parallel and perpendicular fields, respectively, the intergranular lower critical fields are no higher than 0.15 $\mathrm{kA} / \mathrm{m}(1.9 \mathrm{Oe})$ and $0.3 \mathrm{kA} / \mathrm{m}(3.8 \mathrm{Oe})$ at $76 \mathrm{~K}$ and 0.5 $\mathrm{kA} / \mathrm{m}(6.3 \mathrm{Oe})$ and $1.3 \mathrm{kA} / \mathrm{M}(16.3 \mathrm{Oe})$ at $4 \mathrm{~K}$.

[Contact: Robert J. Loughran, (303) 497-3547]

Moreland, J., Rice, P., Russek, S.E., Jeanneret, B., Roshko, A., Ono, R.H., and Rudman, D.A., Scanning Tunneling Microscopy of the Surface Morphology of $\mathrm{YBa}_{2} \mathrm{Cu}_{3} \mathrm{O}_{\mathbf{x}}$ Thin Films Between $300 \mathrm{~K}$ and 76 K, Applied Physics Letters, Vol. 59, No. 23, pp. 3039-3041 (2 December 1991).

Scanning tunneling microscopy (STM) images of $\mathrm{YBa}_{2} \mathrm{Cu}_{3} \mathrm{O}_{\mathrm{x}}(\mathrm{YBCO})$ thin films show different growth habits depending on the deposition method and substrate material. In particular, we present images of YBCO films sputter deposited onto $\mathrm{MgO}$ and $\mathrm{SrTiO}_{3}$ and laser ablated onto $\mathrm{LaAlO}_{3}$. Both screw dislocation and ledge growth are observed. The STM morphology of YBCO films sputtered onto $\mathrm{MgO}$ substrates is investigated as a function of temperature. We find that at room temperature growth steps have an anomolously large apparent height which decreases with decreasing temperature, approaching the expected value for one unit cell of $1.2 \mathrm{~nm}$ at $76 \mathrm{~K}$. Presumably, this phenomenon reflects changes in either the surface tunneling barrier or tunneling density of states upon cooling.

[Contact: John Moreland, (303) 497-3641]

Shi, D., Salem-Sugui, S., Jr., Wang, Z., Goodrich, L.F., Dou, S.X., Liu, H.K., Guo, Y.C., and Sorrell, C.C., Critical Currents in Silver-Sheathed $(\mathrm{Bi}, \mathrm{Pb})_{2} \mathrm{Sr}_{2} \mathrm{Ca}_{2} \mathrm{Cu}_{3} \mathrm{O}_{10-y}$ Superconducting Tapes, Applied Physics Letters, Vol. 59, No. 24, pp. 31713173 (9 December 1991).

Nearly 95 vol\% of the $110-\mathrm{K}$ superconducting phase was formed by lead doping in a $\mathrm{Bi}-\mathrm{Sr}-\mathrm{Ca}-\mathrm{Cu}-\mathrm{O}$ system. The processed $110-\mathrm{K}$ superconducting powders were used to produce long silver-sheathed tapes with a highly textured microstructure by rolling and prolonged sintering. The transport critical-current density was measured at $4.2 \mathrm{~K}$ to be $0.7 \times 10^{5} \mathrm{~A} / \mathrm{cm}^{2}$ at zero field and $1.6 \times 10^{4} \mathrm{~A} / \mathrm{cm}^{2}$ at $12 \mathrm{~T}$ for $\mathrm{H}$ parallel to the plane defined by the $a$ and $b$ axes. At $77 \mathrm{~K}$, the critical-current density reached a value of $\sim 1 \times 10^{4} \mathrm{~A} / \mathrm{cm}^{2}$ at zero field for $\mathrm{H}$ parallel to the plane defined by the $\mathrm{a}$ and $\mathrm{b}$ axes and gradually decreased to $419 \mathrm{~A} / \mathrm{cm}^{2}$ at $1 \mathrm{~T}$. Excellent grain alignment in the $a-b$ plane led to a greatly improved critical-current density under a magnetic field. The relationship between the transport properties and the microstructure of the tapes is discussed.

[Contact: Loren F. Goodrich, (303) 497-3143]

\section{Other Electrical Systems Topics}

\section{Released for Publication}

Martzloff, F.D., and Perry, A.G., Annotated Bibliography - Diagnostic Methods and Measurement Approaches to Detect Incipient Defects Due to Aging of Cable, to be published as U.S. Nuclear Regulatory Commission NUREG/CR 5811. [Previously published as NISTIR 4485 (July 1991).]

Open-literature papers and some limited distribution documents were reviewed in a search to identify 
promising approaches to the in-situ detection of incipient defects in nuclear power plant cables. The search was extended to the topics of detection of any defect, to radiation effects, and to basic considerations on partial discharges. This report presents a review of 150 papers that appeared significant from their title, but many of which were found not applicable upon close review. A compilation of 850 references cited in the reviewed papers is included in this paper.

[Contact: Francois D. Martzloff, (301) 975-2409]

\section{ELECTROMAGNETIC INTERFERENCE}

\section{Conducted}

\section{Recently Published}

Fenimore, C., and Martzloff, F.D., Validating Surge Test Standards by Field Experience: High-Energy Tests and Varistor Performance, Conference Record of the 1990 IEEE Industry Applications Society Annual Meeting, Seattle, Washington, October 7-12, 1990, pp. 1968-1974 (1990).

New, high-energy surge tests are emerging in IEEE and IEC standards. Field experience offers a valuable criterion for validating or invalidating proposed standards. A proposal under consideration by the IEC involves so much energy that a varistor of the voltage rating commonly used in protecting load equipment, if subjected to this test, would almost certainly fail. Yet, reported varistor failure rates do not reflect such a situation. Thus, a re-examination of the premises that led to the proposed test specifications appears necessary. Proposals for high-energy tests as additional waveforms in the new version of IEEE C62.41, on the other hand, lead to current and energy levels that do not place typical varistors in immediate jeopardy. Thus, they appear more consistent with field experience.

[Contact: Charles Fenimore, (301) 975-2428]

\section{Lai, J-S., and Martzloff, F.D., Coordinating Cascaded} Surge Protection Devices: High-Low Versus LowHigh, Proceedings of the 1991 IEEE Industry Applications Society Annual Meeting, Dearborn, Michigan, September 28-October 4, 1991, Vol. II, pp. 1812-1819.

Cascading surge-protective devices between the service entrance of a building and the place near sensitive equipment within the building are intended to ensure that each device shares the surge stress in optimum manner to achieve reliable protection of equipment against surges impinging from the utility supply. However, depending upon the relative clamping voltages of the two devices, their separation distance, and the waveform of the impinging surges, the coordination may or may not be effective. This paper provides computations with experimental verification of the energy deposited in the devices for a matrix of combinations of these three parameters. Results show effective coordination for some combinations and ineffective for some others, a result that should reconcile contradicting findings reported by different authors making different assumptions. From these results, improved coordination can be developed by application standards writers and system designers. [Contact: Francois D. Martzloff, (301) 975-2409]

Martzloff, F.D., A Standard for the 90s: IEEE C62.41 Surges Ahead [original title: IEEE Guide on Surge Voltages Upgraded to Recommended Practice], Compliance Engineering, Vol. 8, No. 5, pp. 27-ff (Fall 1991).

After ten years of use as a guide, a revision has been completed and published as an IEEE Recommended Practice: Surge Voltages in Low-Voltage AC Power Circuits. This article appears in a trade magazine circulated to writers, users, and enforcers of standards on electromagnetic compatibility, in order to give them a preview of the forthcoming IEEE document. [Contact: Francois D. Martzloff, (301) 975-2409]

$\underline{\text { Radiated }}$

\section{Released for Publication}

Allen, O.E., Hill, D.A., and Ondrejka, A.R., TimeDomain Antenna Characterizations.

The standard method of describing an antenna's transmit and receive response is through the many well-defined and accepted frequency-domain antenna characterizations. The frequency-domain characterizations are based on the antenna being excited by a monochromatic signal. When an antenna is designed to transmit and receive signals whose frequency content extends over a wide band, these characterizations become much more cumbersome. A set of timedomain characterizations which can efficiently de- 
scribe such wide-band antennas is proposed in this paper. The experimentally measured responses of a transverse electromagnetic horn antenna is used to evaluate the utility of these characterizations. Comparisons are made between each antenna's frequencydomain response and their time-domain characterizations. The comparisons show that the time-domain characterizations can provide significant insight into the antenna's behavior as well as providing a means to accurately compare two or more different antennas. [Contact: David A. Hill, (303) 497-3472]

Kanda, M., and Ondrejka, A.R., An Ultra Broadband and Non-Dispersive Sensor for the Measurement of Time-Domain Signals, to be published in the Proceedings of the '91 Symposium on Electromagnetic Security for Information Protection, Rome, Italy, November 21-22, 1991.

This paper reviews the ultra broadband and nondispersive antenna for the measurements of timedomain signals. The resistively-loaded TEM horn with the active cross-over network has a nearly constant amplitude and phase response from $2 \mathrm{kHz}$ to $800 \mathrm{MHz}$. The upper frequency response, up to 1 $\mathrm{GHz}$, is limited by the active cross-over network. The antenna transfer function is on the order of $-22 \mathrm{~dB}$ relative to $1 \mathrm{~V}$ output 0 for $1 \mathrm{~V} / \mathrm{m}$.

[Contact: Motohisa Kanda, (303) 497-5320]

\section{Randa, J.P., Correction Factor for Nonplanar Inci- dent Field in Monopole Calibrations.}

In calibrating large monopole antennas, the length of the antenna can be comparable to the separation distance. In that case, there is a significant variation in both the magnitude and the phase of the incident field along the length of the antenna being calibrated. This paper presents an expression for a correction factor to account for this effect. We evaluate the correction factor for some representative cases and present some guidelines for when this factor should be taken into account. The effect can exceed $1.0 \mathrm{~dB}$ in some practical cases.

[Contact: James P. Randa, (303) 497-3150]

Recently Published

Crawford, M.L., and Riddle, B.F., A Proposed TEM Driven Mode-Stirred Chamber for Large System Radiated EMC/V Testing, $10 \mathrm{kHz}$ to $40 \mathrm{GHz}$, Pro- ceedings of the Electromagnetic Compatibility Symposium, Zurich, Switzerland, March 12-14, 1991, pp. 431-437.

This paper describes work in progress at the National Institute of Standards and Technology to develop a single, integrated facility for whole system electromagnetic susceptibility/vulnerability (EMC/V) testing over the frequency range of $10 \mathrm{kHz}$ to $40 \mathrm{GHz}$. The facility will consist of a large shielded enclosure, $13.1 \mathrm{~m}$ by $24.1 \mathrm{~m}$ by $38.7 \mathrm{~m}$ in size, configured as a TEM transmission line-driven, mode-stirred chamber. The anticipated test volume is $7 \mathrm{~m}$ by $16 \mathrm{~m}$ by $30 \mathrm{~m}$. TEM test fields are generated in the chamber at frequencies below multimode cutoff. The paper discusses a proposed design, advantages and limitation, the theoretical basis for the concept, and the experimental approach for using such a facility. Results of an evaluation of a 1/10 scale model chamber $(1.3 \mathrm{~m}$ by $2.4 \mathrm{~m}$ by $3.9 \mathrm{~m})$ are given.

[Contact: Myron L. Crawford, (303) 497-5497]

Koepke, G.H., Driver, L.D., Cavcey, K.H., Masterson, K.D., Johnk, R., and Kanda, M., Standard Spherical Dipole Source, NIST Technical Note 1351 (December 1991).

This report describes the development of a standard spherical dipole transmitter that operates from 10 to $1000 \mathrm{MHz}$. The report includes the complete electronic, mechanical, optical, and theoretical details that are necessary to construct this device and predict the electromagnetic fields which are radiated in simple environments.

[Contact: Galen H. Koepke, (303) 497-5766]

Ma, M.T., Larsen, E.B., and Crawford, M.L., Electromagnetic Fields with Arbitrary Wave Impedances Generated Inside a TEM Cell, IEEE Transactions on Electromagnetic Compatibility, Vol. 33, No. 4, pp. 358-362 (November 1991).

Standard electromagnetic fields having a wide range of wave impedances can be generated inside a TEM cell for rf susceptibility testing purposes. We propose to achieve this by (a) exciting both ports of the TEM cell with the desired amplitudes and phases, or (b) exciting only one port, as usual, and terminating the other port with an unmatched load impedance. Both theoretical expressions and experimental results are 
presented in this paper.

[Contact: Mark T. Ma, (303) 497-3800]

\section{ADDITIONAL INFORMATION}

\section{Lists of Publications}

DeWeese, M.E., Metrology for Electromagnetic Technology: A Bibliography of NIST Publications, NISTIR 3972 (August 1991).

This bibliography lists the publications of the personnel of the Electromagnetic Technology Division of NIST in the period from January 1970 through publication of this report. A few earlier references that are directly related to the present work of the Division are included.

[Contact: Sarabeth Moynihan, (303) 497-3678]

Lyons, R.M., and Gibson, K.A., A Bibliography of the NIST Electromagnetic Fields Division Publications, NISTIR 3973 (August 1991).

This bibliography lists publications by the staff of the National Institute of Standards and Technology's Electromagnetic Fields Division for the period from January 1970 through August 1991. Selected earlier publications from the Division's predecessor organizations are included.

[Contact: Kathryn A. Gibson, (303) 497-3132]

Palla, J.C., and Meiselman, B., Electrical and Electronic Metrology: A Bibliography of NIST Electricity Division's Publications, NIST List of Publications 94 (January 1992).

This bibliography covers publications of the Electricity Division, Center for Electronics and Electrical Engineering, NIST, and of its predecessor sections for the period January 1968 to December 1991. A brief description of the Division's technical program is given in the introduction.

[Contact: Jenny C. Palla, (301) 975-2220]

Walters, E.J., Semiconductor Measurement Technology, 1990-1991, NiST List of Publications 103 (April 1992) and Semiconductor Measurement Technology, 1962-1989, NIST List of Publications 72 (March 1990).

The bibliography provides information on technology transfer in the field of microelectronics at NIST for the calendar years 1990 and 1991. Publications from groups specializing in semiconductor electronics are included, along with NIST-wide research now coordinated by the NIST Office of Microelectronics Programs which was established in 1991. Indices by topic area and by author are provided. Earlier reports of work performed during the period from 1962 through December 1989 are provided in NIST List of Publications 72.

[Contact: E. Jane Walters, (301) 975-2050]

\section{New NIST Research Material}

NIST has announced the availability of Research Material 8458, a well-characterized artificial flaw used as an artifact standard in eddy current nondestructive evaluation (NDE). The new Research Material (RM) is the outcome of work carried out by the Electromagnetic Technology Division to address the need for calibration standards for eddy-current NDE, for example, as used to detect fatigue cracks in aircraft structures. The RM flaw is produced in an annealed aluminum alloy block by first indenting the block and then compressively deforming the resulting notch until it is tightly closed. The next operation is to restore a flat finish to the block face, after which the block is heat treated to the original temper. The controlled flaw has been named the "CDF notch," after its inventors (listed on patent application) Thomas E. Capobianco (Electromagnetic Technology Division), William P. Dube (Division 832), and Ken Fizer (Naval Aviation Depot, NAS Norfolk, Virginia).

In the past, the challenge has been to manufacture artificial flaws that closely simulate the mechanical properties of fatigue cracks. Currently used artifacts include electrical-discharge-machined and saw-cut notches, both of which are relatively poor representations of fatigue cracks as their widths are too great. The Division-developed method provides notches that can be made controllably in a variety of geometries, have known dimensions, with widths that are narrow enough to provide an acceptable representation of fatigue cracks.

An NIST Research Material is not certified by NIST, but meets the International Standards Organization definition of "a material or substance one or more properties of which are sufficiently well established to be used in the calibration of an apparatus, the assess- 
ment of a measurement method, or for assigning values to materials." The documentation issued with RM 8458 is a "Report of Investigation." Contact: technical information - Fred Fickett, (303) 497-3785; order information - Standard Reference Materials Program, (301) 975-6776.

Continuing Production-Expanded Capability Standard Reference Materials

The Semiconductor Electronics Division announces the continuing production of three thicknesses and the addition of two new thicknesses for the Standard Reference Material (SRM) for ellipsometrically derived thickness and refractive index of a silicon dioxide film on silicon. For sale to the public through the NIST Standard Reference Material Program [(301) 975-6776], the following three individual oxide thicknesses continue to be available: $50 \mathrm{~nm}$ (SRM 2531), $100 \mathrm{~nm}$ (SRM 2532), and $200 \mathrm{~nm}$ (SRM 2533). Recently, two new thicknesses, 25 nm (SRM 2534) and a limited number of 14-nm prototypes (SRM 2535), were added to the availability list.

SRMs 2531, 2532, and 2533, originally released as SRM 2530-1, 2530-2, and 2530-3, were developed in response to the industry's need to evaluate the accuracy of ellipsometers and other thin-film thicknessmonitoring instruments. The scope of these SRMs has now expanded with the recent issuance of the 25$\mathrm{nm}$ and 14-nm oxide thicknesses so they have application as thickness standards for use in research as well as in semiconductor fabrication production lines.

Each SRM unit, consisting of a 76-mm (3-in) diameter silicon wafer on which a uniform silicon dioxide layer has been grown, is individually measured and certified over a 5 -mm diameter area in the center of the wafer for the ellipsometric parameters delta, $\Delta$, and psi, $\psi$, at the vacuum wavelength $\lambda=633.0 \mathrm{~nm}$ using the High-Accuracy Ellipsometer built at NIST. Each SRM is also certified for the derived values for the thicknesses and indices of refraction of both layers of a two-layer optical model of an oxide film on a single-crystal silicon substrate.

[Contact: Barbara J. Belzer, (301) 975-2248]

$\underline{\text { Recently Issued Standard Reference Materials }}$

The Microelectronics Dimensional Metrology Group of the Precision Engineering Division announces the release of two Standard Reference Materials (SRMs) for calibrating optical microscopes used to measure linewidths on photomasks. Each SRM consists of a $63.5 \times 63.5 \times 1.5 \mathrm{~mm}(2.5 \times 2.5 \times 0.060 \mathrm{in})$ photomask patterned with chromium lines of widths in the range of 0.9 to $10.8 \mu \mathrm{m}$. SRM 475, patterned with antireflecting chromium on a quartz substrate, is being reissued after being out of production for almost four years. SRM 476, a new SRM, is patterned with bright chromium on a borosilicate substrate.

In addition to isolated opaque lines on a clear background and isolated clear lines on an opaque background, these SRMs contain opaque line pairs for calibrating the length scale of optical microscopes, adjacent clear and opaque lines of approximately equal widths for setting the line-to-space ratio (contrast) on video image-scanning instruments, and features with 10 approximately equally spaced opaque lines for checking the linearity of measurement systems (e.g., the magnification as a function of position over the field of view).

The certified linewidth and spacing values were determined from measurements made with the NIST automated linewidth measurement system. The uncertainty of the linewidth measurements is $0.081 \mu \mathrm{m}$ or less for SRM 475 and $0.064 \mu \mathrm{m}$ or less for SRM 476. The dominant contribution to this uncertainty is the nonvertical geometry of the line edges, and finding a source of photomasks with better edge geometry would lead to considerable improvement in the calibration uncertainty.

[Contact: James Potzick, (301) 975-3481 or Robert Larrabee, (301) 975-2298]

Emerging Technologies in Electronics ... and Their Measurement Needs, Second Edition

This report assesses the principal measurement needs that must be met to improve U.S. competitiveness in emerging technologies within several fields of electronics: semiconductors, superconductors, magnetics, optical fiber communications, optical fiber sensors, lasers, microwaves, video, and electromagnetic compatibility. The report seeks feedback from industry and Government agencies on the assessment. The feedback will guide the development of NIST programs that provide U.S. industry with new documented measurement methods, new national reference standards to assure the accuracy of those measure- 
ment methods, and new reference data for electronic materials. Copies may be obtained by ordering Report No. PB90-188087/AS (\$23.00 hard copy, $\$ 11.00$ microfiche) from the National Technical Information Service, 5285 Port Royal Road, Springfield, VA 22161, (703) 487-4650.

Transfer of Pulse Waveform Measurements Services to NIST, Gaithersburg, MD

The responsibility for the Special Test Services Provided by NIST for pulse waveform measurements has now been officially transferred to the Electricity Division, Electronic Instrumentation and Metrology Group (811.02) in Gaithersburg, MD. These services include:

\section{Test Number Description of Services}

65100S Impulse Generator Spectrum Amplitude (50 Ohm)

65200S Fast Repetitive Broadband Pulse Parameters (50 Ohm)

65300S Network Impulse Response $\left(\mathrm{S}_{21}\right)$ of Coaxial Networks

65400S Pulse Time Delay through Coaxial Transmission Lines

Service for test number $65400 \mathrm{~S}$ is already available; it is anticipated that the equipment and software necessary for bringing the other waveform measurement services on line will also become available by June 30 , 1992. Please direct specific technical questions concerning these services to Mr. William L. Gans, (301) 975-2502.

\section{2/1993 EEEL CALENDAR}

July 23, 1992 (Gaithersburg, Maryland)

Ion Implant Users Group Meeting. Sponsored by NIST, the next meeting will address the following topics: Simulation and Modeling (Part 1) and Rapid Thermal Processing.

[Contact for general information about the Users Group or this meeting in particular: John Albers, (301) 975-2075]

October 8, 1992 (Gaithersburg, Maryland)

Ion Implant Users Group Meeting. Topics to be discussed at this NIST-sponsored meeting include Simulation and Modeling (Part 2). An afternoon tour of the Semiconductor Electronics Division, SED's Semiconductor Processing Research Facility, the Secondary Ion Mass Spectrometry Facility, and the Reactor's Cold Neutron Research Facility is planned. [Contact: John Albers, (301) 975-2075]

November 9-10, 1992 (Austin, Texas)

Workshop on Process Control Measurements for Advanced IC Manufacturing. In conjunction with SEMICON Southwest, the workshop, cosponsored by ASTM, JEIDA, JESSI, NIST, SEMATECH, SEMI, and SRC, will begin with overviews of the status of process control measurements in silicon device fabrication. Working sessions will consider the critical process control measurement issues in the following areas: film deposition, contamination, implant, etching, oxidation/diffusion, lithography, and materials. Standards development meetings will be held concurrently by ASTM Committee F-1 on Electronics and a selected number of SEMI Standards committees.

[Contact Robert I. Scace, (301) 975-4400]

February 2-4, 1993 (Austin, Texas)

9th Annual IEEE Semiconductor, Thermal Measurement and Management Symposium (SEMI-THERM). Sponsored by IEEE CHMT and NIST, SEMITHERM is the premier forum for the exchange of information on thermal management of electronics systems between the academic and industrial communities. The program will address the following topics: analytical and computational modeling; measurement techniques including temperature, fluid flow, and thermal-mechanical properties; and thermal reliability screening and testing.

[Contact: David Blackburn, (301) 975-2053]

\section{EEEL SPONSORS}

National Institute of Standards and Technology U.S. Air Force

McClelland Air Force Base; Newark Air Force

Station; Rome Air Development Center; Space \& Missile Organization; Wright-Patterson Air Force Base; SAF/FMBMB, Pentagon

U.S. Army

Dugway Proving Ground; Fort Belvoir; Fort Huachuca; Harry Diamond Laboratory; Strategic 
Defense Command; AVRADCOM (Aviation)

Department of Commerce

NOAA; Census

Department of Defense

Advanced Research Projects Agency; Combined Army/Navy/Air Force (CCG); National Security

Agency; Oak Ridge National Laboratory

Department of Energy

Energy Systems Research; Fusion Energy; Basic

Energy Sciences; High Energy \& Nuclear Physics

Department of Justice

Law Enforcement Assistance Administration; FBI

U.S. Navy

Naval Sea Systems Command; Weapons Support
Center/Crane; Office of Naval Research; Naval Air Systems Command; Naval Air Engineering Center; Naval Research Laboratory; Naval Aviation Depot; Naval Command, Control, and Ocean Surveillance Center, RDT\&E Center; Naval Air Test Center; Naval Survace Warfare Center

National Aeronautics and Space Administration NASA Headquarters; Goddard Space Flight Center; Lewis Research Center

Nuclear Regulatory Commission

Department of Transportation

National Highway Traffic Safety Administration MIMIC Consortium

Various Federal Government Agenices 

NIST-114A
(REV. 3-90)
U.S. DEPARTMENT OF COMMERCE
NATIONAL INSTITUTE OF STANDARDS AND TECHNOLOGY

\section{BIBLIOGRAPHIC DATA SHEET}

1. PUBLICATION OR REPORT NUMBER

NISTIR 4850

2. PERFORMING ORGANIZATION REPORT NUMBER

3. PUBLICATION DATE

July 1992

4. TITLE AND SUBTITLE

Electronics and Electrical Engineering Laboratory Technical Progress Bulletin Covering Laboratory Programs, October to December 1991, with 1992/1993 EEEL Events Calendar

5. AUTHOR(S)

J. A. Gonzalez, compiler

6. PERFORMING ORGANIZATION (IF JOINT OR OTHER THAN NIST, SEE INSTRUCTIONS)

U.S. DEPARTMENT OF COMMERCE

NATIONAL INSTITUTE OF STANDARDS AND TECHNOLOGY

GAITHERSBURG, MD 20899

7. CONTRACT/GRANT NUMBER

8. TYPE OF REPORT AND PERIOD COVERED

October-December 1991

9. SPONSORING ORGANIZATION NAME AND COMPLETE ADDRESS (STREET, CITY, STATE, ZIP)

10. SUPPLEMENTARY NOTES

All technical information included in this document has been approved for publication previously.

11. ABSTRACT (A 200-WORD OR LESS FACTUAL SUMMARY OF MOST SIGNIFICANT INFORMATION. IF DOCUMENT INCLUDES A SIGNIFICANT BIBLIOGRAPHY OR LITERATURE SURVEY, MENTION IT HERE.)

This is the thirty-seventh issue of a quarterly publication providing information on the technical work of the National Institute of Standards and Technology, Electronics and Electrical Engineering Laboratory. This issue of the EEEL Technical Progress Bulletin covers the fourth quarter of calendar year 1991. Abstracts are provided by technical area for both published papers and papers approved by NIST for publication.

12. KEY WORDS (6 TO 12 ENTRIES; ALPHABETICAL ORDER; CAPITALIZE ONLY PROPER NAMES; AND SEPARATE KEY WORDS BY SEMICOLONS)

antennas; electrical engineering; electrical power; electromagnetic interference; electronics; instrumentation; laser; magnetics; microwave; optical fibers; semiconductors; superconductors

13. AVAILABILTYY

UNLIMITED

FOR OFFICIAL DISTRIBUTION. DO NOT RELEASE TO NATIONAL TECHNICAL INFORMATION SERVICE (NTIS).

ORDER FROM SUPERINTENDENT OF DOCUMENTS, U.S. GOVERNMENT PRINTING OFFICE, WASHINGTON, DC 20402.

ORDER FROM NATIONAL TECHNICAL INFORMATION SERVICE (NTIS), SPRINGFIELD, VA 22161.
14. NUMBER OF PRINTED PAGES

53

15. PRICE

$\mathrm{A} 04$ 



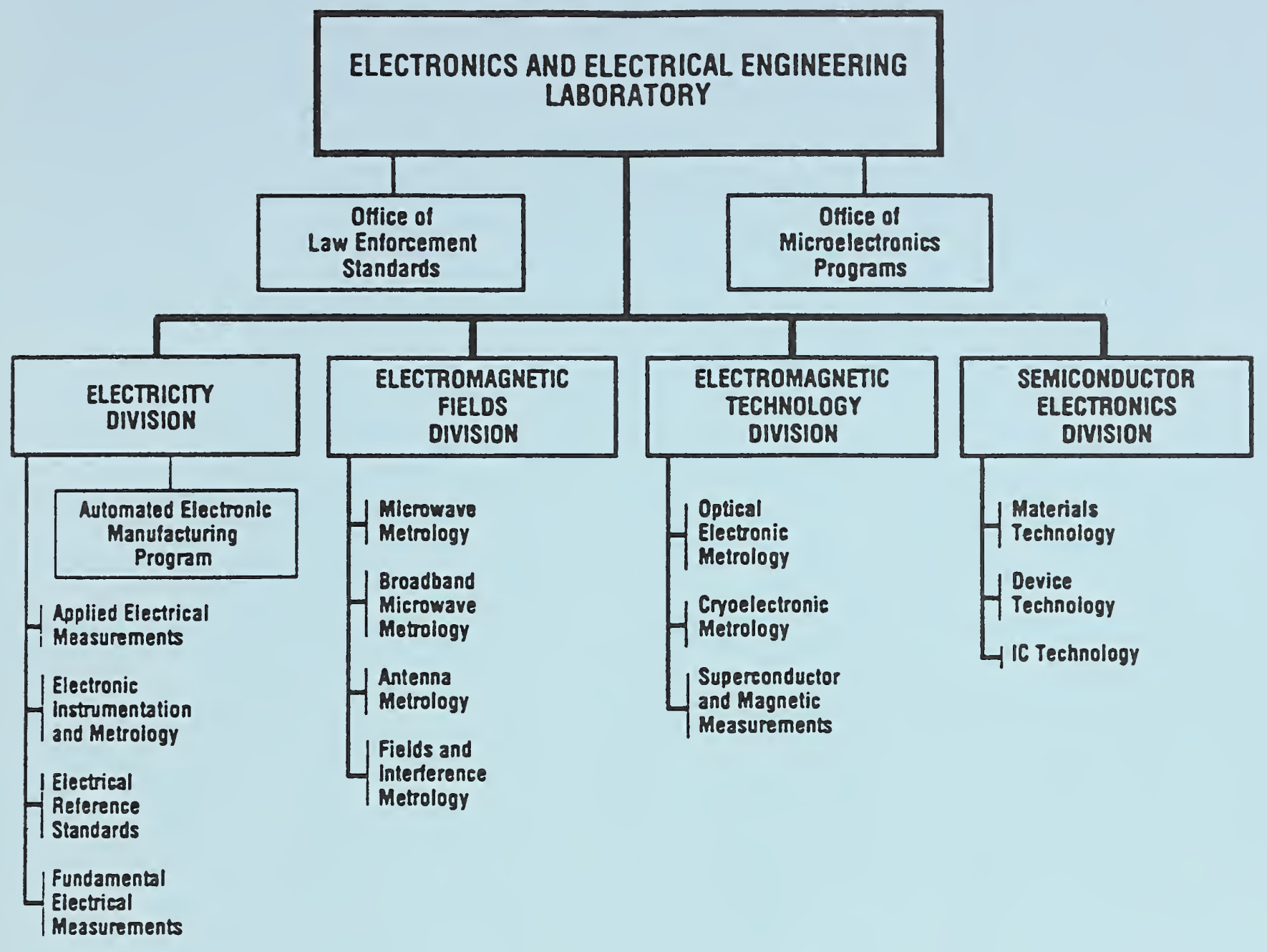

\section{KEY CONTACTS}

Laboratory Headquarters (810)

Office of Microelectronics Programs

Office of Law Enforcement Standards

Electricity Division (811)

Semiconductor Electronics Division (812)

Electromagnetic Fields Division (813)

Electromagnetic Technology Division (814)
Director, Mr. Judson C. French (301) 975-2220

Deputy Director, Dr. Robert E. Hebner (301) 975-2220

Director, Mr. Roben I. Scace (301) 975-4400

Director, Mr. Lawrence K Eliason (301) 975-2757

Chief, Dr. Oskars Petersons (301) $975-2400$

Chief, Mr. Frank F. Oettinger (301) 975-2054

Chief, Dr. Ramon C. Baird (303) $497-3131$

Chief, Dr. Robert A. Kamper (303) 497-3535

\section{INFORMATION:}

For additional information on the Electronics and Electrical Engineering Laboratory, write or call: Electronics and Electrical Engineering Laboratory

National Institute of Standards and Technology

Metrology Building, Room B-358

Gaithersburg, MD 20899

Telephone: (301) $975-2220$ 
